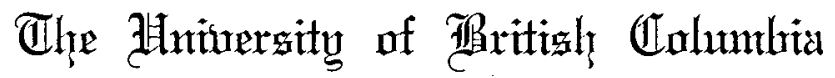

Faculty of Graduate Studies

PROGRAMME OF THE

FINAL ORAL EXAMINATION

FOR THE DEGREE OF

DOCTOR OF PHILOSOPHY

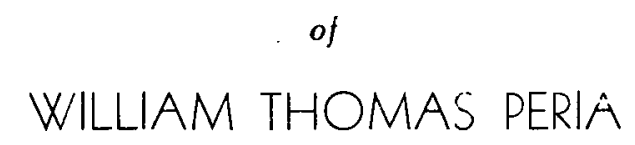

M. A. Sc., University of British Columbia, 1951

TUESDAY, AUGUST 6th, 1957, at 2:30 p.m.

IN ROOM 300, PHYSICS BUILDING

\author{
COMMITTEE IN CHARGE \\ DEAN G. M. Shrum, Chairman
}
A. M. CROOKER
J. NORRIS
K. C. MANN
F. NOAKES
R. E. BURGESS
R. D. JAMES
J. B. GUNN
G. B. PORTER

External Examiner Dr. EUGENE B. HENSLEY

University of Missouri 


\title{
OPTICAL ABSORPTION AND PHOTOCONDUCTIVITY IN MAGNESIUM OXIDE CRYSTALS
}

\begin{abstract}
The purpose of this investigation was the determination of the nature of certain imperfections in magnesium oxide crystals. Optical absorption and photoconductivity spectra of specimens cleaved from a number of larger pieces were measured. The effect of vacuum heating, of nonstoichiometry and of ultraviolet and $\mathrm{x}$-ray irradiation were investigated. The nature of the imperfections could not be inferred from the experimental results but an energy level diagram consistent with all the data has been deduced.
\end{abstract}

A comparison of the present work with pertinent data from the literature is presented and a basic error in previous photoconductivity measurements is pointed out.

A method for the determination of the sign of the charge carriers excited during photoconductivity measurements is described. 


\section{GRADUATE STUDIES}

Field of Study: Physics

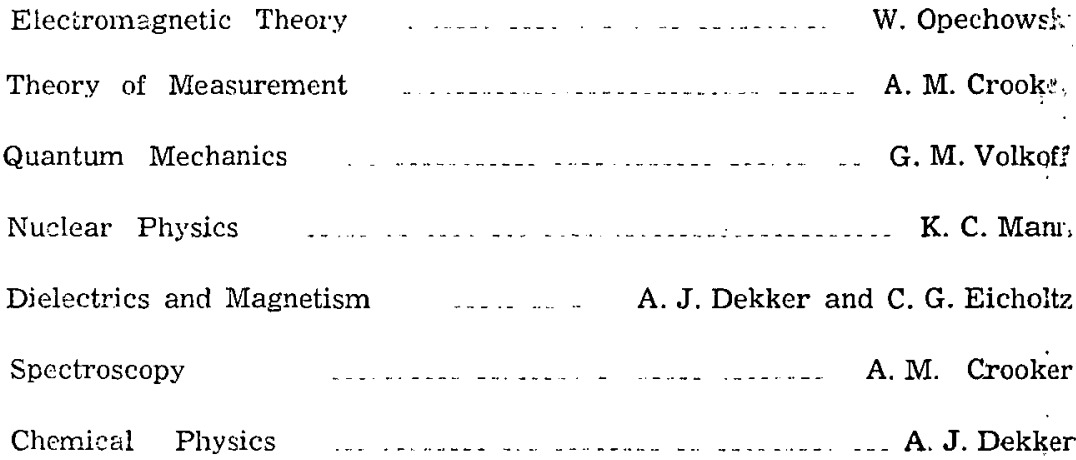

Other Studies:

Advanced Quantum Mechanics

L. Teng

Mathemetical Foundations of Statistical Mechanics

P. C. Rosenbloom 
OPTICAL ABSORPTION AND PHOTOCONDUCTIVITY

IN MAGNESIUM OXIDE CRYSTALS

by

William Thomas Peria

M.A. Sc. University of British Columbia, 1951

A THESIS SUBMITTED IN PARTIAL FULFILLMENT OF THE REQUIREMENTS FOR THE DEGREE OF DOCTOR OF PHILOSOPHY

in the Department

of

Physics

We accept this thesis as conforming to the required standard

THE UNIVERSITY OF BRITISH COLUMBIA April, 1957 
In presenting this thesis in partial fulfilment of the requirements for an advanced degree at the University of British Columbia, I agree that the Library shall make it freely available for reference and study. I further agree that permission for extensive copying of this thesis for scholarly purposes may be granted by the Head of my Department or by his representative. It is understood that copying or publication of this thesis for financial gain shall not be allowed without my written permission. Department of Phe University of British Columbia, Vanc ouver 8 , Canada.

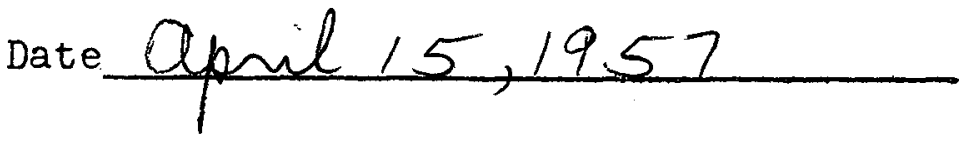




\section{ABSTRACT}

The purpose of this investigation was the determination of the nature of certain imperfections in magnesium oxide crystals. Optical absorption and photoconductivity spectra of specimens cleaved from a number of larger pieces were measured. The effects of vacuum heating, of non-stoichiometry and of ultraviolet and $\mathrm{X}$-ray irradiation were investigated. The nature of the imperfections could not be inferred from the experimental results but an energy level diagram consistent with all the data has been deduced.

A comparison of the present work with pertinent data from the literature is presented and a basic error in previous photoconductivity measurements is pointed out.

A method for the determination of the sign of the charge carriers excited during photoconductivity measurements is described. 
Section

$\therefore$

A

B

Introduction . . . . . . . . . 1
Experimental Details. . . . . 5
Results

B.1* Optical Absorption Measurements . . 10

2 Analysis of Photoconductivity Data . . 11

3 Dependence of Photocurrent on Light Intensity and Electric Field . . . 14

4 Evidence that Photocurrents are a Bulk Effect ........... 14

5 Space Charge Formation. . . . . 15

6 Properties of Crystals as Received . . 16

7 Heat Treatment Studies... . . 20

8 Excess Magnesium Crystals ... . . 21

9 The Effect of Ultraviolet

Irradiation ......... . 22

10 The Effect of X-Irradiation . . . 23

11 Sign of the Charge Carriers . . . . 26

C Discussion . . . . . . . . . . 29

C.l Optical Absorption . . . . . . . 29

2 Discussion of the Factors Involved in the Photoconductive Yield.... 30

3 The Dependence of the Photocurrent on Light Intensity and Electric Field ............. 33

4 Evidence that the Photocurrents are Due to a Bulk Effect....... 37

5 Formation of Space Charge Fields... 38

* Sections $B$ and $C$ are divided into 11 subsections, each in such $a$. way that the results described in B.l are discussed in C.I and so on. 
TABLE OF CONTENTS (continued)

Section

Page

C.6 Properties of Crystals as Received . . . 43

7 Heat Treatment Studies ....... 47

8 Excess Mg Crystals ........ 50

9 Ultraviolet Irradiation ........ 52

$10 \mathrm{X}$-Ray Irradiation ........ 56

11 Sign of the Charge Carriers . . . . 64

D Conclúsions ... . . . . . . . 69 
Schematic Arrangement of the Photoconductivity Apparatus

Mounting of the Crystal for Photoconductivity

Measurements

Development and Removal of Space Charge

Typical Absorption Spectra of MgO Crystals as

Received

Typical Photoconductivity and Absorption Spectra

Resolution of a Typical Photoconductivity Spectrum into

Two Gaussian Bands

Effect of Heat Treatment on the Background Absorption

Photoconductivity and Absorption of Crystals Heated

in Vacuum at Different Temperatures

Absorption Spectrum Due to Heating in Magnesium Vapor 9

The Effect of Excess Magnesium on the Background

Absorption of $\mathrm{MgO}$

The Effect of Excess Magnesium on the Background Absorption of $\mathrm{MgO}$

The Effect of Excess Magnesium on the Background Absorption of $\mathrm{MgO}$

Effect of Excess Mg on Photoconductivity

Optical Activation of Photoconductivity

Effect of Ultraviolet Irradiation on Photoconductivity in $\mathrm{MgO}$

Increase in Optical Density by Irradiation in

5 ev Band

Induced Optical Absorption Due to Irradiation in 4 ev Band

Buildup of X-Ray Induced Absorption 18

Absorption Spectra of X-Rayed Crystals 19

Decay of X-Ray Induced Absorption 20

Photoconductivity in X-Rayed MgO 21 
INDEX TO FIGURES (continued)

Title

Figure No.

Photoconductivity in X-Rayed MgO

Effect of $X$-Irradiation on the Photoconductivity of Ultraviolet Activated $\mathrm{MgO}$

Bleaching of $X-$ Ray Induced Absorption at 4.2 ev by 2.3 ev Quanta

Bleaching of X-Ray Induced Absorption at $2.3 \mathrm{ev}$ by $2.3 \mathrm{ev}$ Quanta

Bleaching of $\mathrm{X}$-Ray Induced Photoconductivity by 2.3 ev Quanta

Determination of the Sign of Charge Carriers Excited in $4 \mathrm{ev}$ Band

Determination of the Sign of Charge Carriers Excited in 5 ev Band

Determination of the Sign of Charge Carriers in an $\mathrm{X}$-Rayed CrystaI

Possible Mechanisms for the Loss of Free Carriers 30

Proposed Energy Level Schemes for MgO

Thermal Decay of X-Ray Induced Ultraviolet Absorption at Room Temperature

Idealized Field Distributions Illustrating the Method of Determining the Sign of the Charge Carriers 


\section{INDEX TO TABLES}

Table No.

Title

Page

I

Absorption and Photoconductivity at $5.0 \mathrm{ev}$

15

II

Photoconductive Yield of a Number of Specimens

III

Fractional Change in Photoconductive Yield by Bleaching with $2.3 \mathrm{ev}$ Quanta

IV

Calculated Thermal Ionization Energies of the Shallow Electron Traps 


\section{ACKNOWLEDGEMENTS}

Support for this work was provided by the United States Army Signal Corps through contracts with the University of Minnesota Electron Tube Research Laboratory. The author wishes to express his gratitude to Professor W. G. Shepherd, director of the Laboratory, for the provision of facilities and for helpful discussions. Thanks are due also to.professor A. J. Dekker whose encouragement enabled the writer to proceed to graduate study and who discussed all phases of the work. B. V. Haxby, R. G. Lye, R. W. Soshea and P. Wargo have provided a beneficial exchange of ideas.

Appreciation to Joan Theresa Peria for her encouragement and forbearance is hereby affectionately extended. 


\section{INTRODUCTION}

The increasing importance of semiconductors and insulators in the modern technology need hardly be emphasized. Solid state devices are finding more and more application in most endeavors involving the use of electronic circuits. As is always the case, increased application calls for increased understanding of the physical properties of the materials involved. Since many of the useful and interesting properties of these solids stem from the presence of deviations of the lattice from strict periodicity, a study of such solids nearly always consists of a study of the lattice imperfections, whethe $r$ they occur naturally or are purposely introduced.

Deviations from periodicity can introduce, into the forbidden energy region characteristic of the unperturbed lattice, localized energy levels, i.e., the imperfect solid has some eigenstates whose eigenvalues lie in the normally forbidden region of energy and whose eigenfunctions are localized in the region of the imperfection rather than extending throughout the whole lattice. The determination of the location of such levels on the energy scale is a first step in the study of the nature of the corresponding imperfections. However, it is possible that the investigation of the energy level scheme for a particular imperfect solid may have some importance beyond the understanding of the physical properties of this solid alone. One has only to consider the vital role that the determination of atomic and nuclear energy level schemes has played in the development of physics to realize the 
significance of this statement. Of course, a knowledge of the scheme alone does not permit the deduction of the nature of the imperfections. Inferences as to the constitution of a particular imperfection may be made in a number of ways;

(1) By studying the properties of this imperfection by itself and comparing the observed behavior with the properties predicted by various theoretical models,

(2) By observing reactions of the imperfections among themselves and with other types,

(3) By introducing imperfections in such a way as to favor the production of a specific type.

The first group of compounds to receive a thorough investigation of its structure sensitive (i.e., imperfectioncontrolled) properties was the monovalent ionic alkali halide group. These materials were studied extensively by the Gottingen school under Pohl, beginning about 1930. These experiments might be regarded as the beginning of semiconductor physics. Many of the concepts employed in current research on semiconductors arose in connection with the work on these alkali halides. Of course many other groups of solids are currently receiving much attention. Of these the group which is probably most closely related to the alkali halides is the group of alkaline earth oxides, i.e., divalent ionic compounds. In addition to the interest in these compounds because of their analogy to the better known monovalent ionic compounds, they all have important.device applications. Barium and strontium oxides are used in thermionic cathodes while magnesium oxide is a 
very efficient secondary electron emitter. Of these three compounds magnesium oxide is by far the easiest to obtain and work with in single crystal form.

A concerted effort to understand the secondary emitting characteristics of this material has been underway in the Electron Tube Laboratory of the University of Minnesota for several years. Because it was believed that the secondary emission is influenced by certain types of imperfections, studies of the electrical and optical properties of these imperfections have been carried out as an integral part of this program.

As mentioned previously, crystalline imperfections lead to localized energy levels lying in the forbidden energy gap. Excitation of electrons to or from these levels leads of course to optical absorption bands which would not be present in the perfect crystal. The absorption processes may lead to free charge carriers (electrons or holes) either directly or by subsequent thermal steps. In either case photoconductivity measurements may lead to additional information which will aid in the construction of an energy level diagram. It is also conceivable that imperfections not detectable in optical absorption may be detected in photoconductivity (or vice versa) since the limits of detection in the two cases are governed by different properties of the center and the host crystal. This will be discussed in more detail below. The work to be described in this thesis was aimed at a 
determination of the nature of certain of the color centers commonly observed in MgO crystals, by the measurement of optical absorption and photoconductivity of specimens treated in various ways. Insofar as an unambiguous energy level scheme has not been determined, not even the first step in the original purpose has been achieved. On the other hand, by proceeding in each of the three fashions enumerated previously, it has been possible to make certain inferences concerning the properties of some of the color centers and the relations between them which at the very least, suggest further, more crucial experiments. 


\section{A. EXPERIMENTAL DETAILS}

Unless otherwise noted the crystals used in these experiments were obtained from the Norton Company, Niagara Falls, New York. Only those pieces which showed no visible absorption were used. From these larger pieces thin slabs could be easily cleaved out. These were usually about $5 \times 10 \mathrm{~mm}$ on the faces and from 0.2 to $1.0 \mathrm{~mm}$ thick.

Some of the crystals were heated in vacuum before use. Such heat treatments were carried out in a small furnace operating inside a bell jar. The pressure was usually about $5 \times 10^{-5} \mathrm{~mm} \mathrm{Hg}$. To prevent contamination of the crystal faces during the heat treatment, they were placed in a boat ground from a large MgO crystal and a MgO slab lid was tied on with molybdenum wire.

Specimens were additively colored with Mg by heating them in steel bombs containing Mg metal. Two-chamber bombs with independent temperature controls were used. In this way the temperature of the crystals (in the hotter chamber) and the vapor pressure of the metal could be varied independently. The bombs were assembled in air by means of a tapered conical joint. Evacuation was not necessary since the metal readily combined with the oxygen and nitrogen at the temperatures employed. The crystals were heated at temperatures in the range $1100^{\circ} \mathrm{C}$ to $1350^{\circ} \mathrm{C}$, in $\mathrm{Mg}$ pressures from 1 to $7500 \mathrm{~mm} \mathrm{Hg}$, and for times from 1 to 50 hours. On some occasions the bombs were quenched by dropping into water, on others they were allowed to cool slowly. 
The apparatus employed for the measurement of photoconductivity is shown schematically in Figure 1 . Light from the source A was focused by the 12-inch diameter elliptical aluminum mirror $B$ onto the entrance slit of a Bausch and Lomb grating monochromator $\mathrm{C}$, having a dispersion of 33 angstrom/mm. The monochromator was normally used with slits of $3 \mathrm{~mm}$ or less in width so that the band width was normally about $100 \mathrm{~A}$ or less. At $5 \mathrm{ev}$ this corresponds to an energy range of $0.2 \mathrm{ev}$ in the monochromator output. To remove the higher order dispersions from the monochromator output, sharp-cut glass filters could be inserted at $D$. These were prevented from overheating when necessary by $2 \mathrm{~cm}$ of water between fused quartz plates (E). The output from the monochromator passed through the fused quartz plate $F$, from which a fraction of the beam was focused onto a type 935 photocell, G, by the front surface aluminized mirrox, H. The main portion of the beam was focused onto the crystal $\mathrm{J}$ by means of a second front surface mirror, K. The latter was pivoted so that it could be moved in a horizontal plane by means of micrometer screw, L. All the parts follow ing the exit slit of the monochromator were enclosed in a light-tight box with a fused quartz entrance window. The box could be desiccated when necessary.

Three light sources were employed to cover the energy range from 1.7 to about 5.6 ev. A carbon arc, using a National Carbon Company Type $W$ cored anode, operating at $40 \mathrm{~V}$, $60^{\mathrm{A}}$, could be used over the entire range. More stable output was obtained from a General Electric Company Type AH6 high 
pressure mercury arc $(2.3-4.6 \mathrm{ev})$ or a tungsten lamp $(1.7-2.3 \mathrm{ev})$. At energies greater than $5.0 \mathrm{ev}$ there was an appreciable amount of stray light in the monochromator output. This was accounted for by measuring the decrease in photocurrent and light intensity when a Corning 9700 filter was placed in the bear. This filter cut off the energy at which the measurement was being made but passed practically all the stray radiation.

The photocell $G$ was calibrated in a separate experiment by placing a calibrated thermopile in the sample position and measuring the output of both photocell and thermopile as a function of wavelength. The photocell output was detected by a D.C. amplifier constructed according to the design of Lander ${ }^{l}$ while a Perkin Elmer Model 53 breaker amplifier measured the the rmopile output.

The the rmopile used had a time constant of approximately I sec., so that the calibration procedure would normally have required stability of the light source over periods of several seconds. This condition could not be met when the carbon arc was employed. This difficulty was overcome, however, by placing, in the photocell circuit, a network having an electrical time constant equal to the thermal time constant of the thermopile. Under these conditions a comparison of thermopile and photocell outputs at a given time provides the required calibration, despite fluctuations in the light source. To facilitate the comparison, the two outputs were recorded on a Sanborn Model 64-1300 A multi-channel recorder. 
Due to the lack of sensitivity, the photocell could not be used at quantum energies below $2.30 \mathrm{ev}$. By using the calibrated thermopile the variation of the output of the tungsten lamp with energy was determined. Then under a given set of conditions the intensity could be measured at $2.3 \mathrm{ev}$ using the photocell and the intensity at lower quantum energies calculated from this.

Crystals were prepared for photoconductivity measurements by painting electrodes of air-drying silver paint (DuPont No. 4817) on two of the edges. They were held between Teflon blocks, which were also coated on one face with the same silver paste (Fig. 2).

The electric field was applied to the crystal by a number of $300^{\mathrm{V}}$ dry cells, while the current was measured by a directcoupled feedback amplifier whose first stage was mounted near the crystal. An input resistor of $10^{12}$ ohms could be employed and currents of $5 \times 10^{-15}$ amperes were readily measurable. With this input resistor the time constant of the system was about 2 seconds. The sensitivity and linearity of the system were checked by applying the accurately known voltages from a Rubicon potentiometer to the input.

In all the photocurrent measurements, the exposure of the crystal to light, when the electric field was applied, was kept as small as possible in order to minimize the formation of space charge fields. The light was allowed to fall on the crystal in single "pulses" varying from .05 to about 5 seconds depending on the sensitivity (and hence response time) of the 
apparatus. A magnetically operated shutter was set up to provide the shorter light pulses, but this was ordinarily found to be unnecessary since the currents were small enough to require the use of the largest input resistors. Under these circumstances the longer response time of the amplifier required light flashes of durations easily obtained by means of a manually operated shutter. The flashes were still too short, however, to permit the photocurrent and light intensity to be read from meters. Consequently, both quantities were recorded simultaneously on the previously mentioned recorder.

Aside from the prevention of space charge formation, the use of short pulses of light was desirable because of the poor long-term stability of the carbon arc and the effect of prolonged UV radiation on the photoconductive response. 


\section{B. RESULTS}

\section{B. * Optical Absorption Measurements $^{*}$}

Absorption spectra were measured with a Beckman Model DU quartz spectrophotometer. The data are usually presented in terms of the absorption coefficient, $K$, defined by

$$
I=I_{0} e^{-K d}(1-R)^{2}
$$

where $I_{0}$ and $I$ are the incident and transmitted intensities, respectively, $d$ is the specimen thickness and $R$ is the reflection coefficient. The above expression allows for the losses at the first and second partial relfectionsionly. Since $R$ is small, this approximation is sufficiently accurate for our purposes. $R$ was calculated from the index of refraction data of Strong and Brice ${ }^{2}$, which was extrapolated into the short wavelength region using the Sellmaier equation ${ }^{3}$,

$$
\mu^{2}=\frac{1.945 \lambda 2}{\lambda^{2}-1.251 \times 10^{6}}
$$

where $\mu$ is the index of refraction and $\lambda$ the wavelength expressed in Angstrom units.

Since the cleaved surfaces of the crystals were not perfectly smooth but had a "wavy" appearance, these calculated reflection losses were no doubt too low. Where absorption changes $(\Delta K)$ are presented, this loss, of course, cancels out.

* Sections $B$ and $C$ are divided into 11 subsections each in such a way that the results described in B.I are discussed in C.I and so on. 
On some occasions, the change in optical density $(\Delta D)$ is plotted rather than the change in absorption coefficient. Since

$$
D=\log _{10} \frac{I_{0}}{I}
$$

then

$$
\Delta \mathbb{R}=\frac{2.3}{d} \quad \Delta D
$$

B.2 Analysis of the Photoconductivity Data

For reasons which will become evident in later sections, the photoconductivity data had to be analyzed in a somewhat different fashion from that normally employed. The analysis used is presented below:

Consider a crystal with an absorption coefficient $K(E)$, where $E$ designates the quantum energy. $K$ may be a composite coefficient which defines the total absorption, at a given energy, due to several different absorption processes, $K_{j}(E)$, i.e.,

$$
K(E)=\sum_{i} K_{i}(E)
$$

If the crystal absorbs a fraction, a radiation, the fraction absorbed by optical transitions of the $i$ th kind is $\frac{K_{i}{ }^{a}}{K}$. Let $p_{i}$ be the probability that such a transition leads to a free charge carrier. Then the fraction of the incident radiation which produces free charge carriers, by all possible types of transitions, is

$$
\frac{a}{K} \sum_{i} p_{i} K_{i}
$$


Let the carriers move an average distance $x_{i}$ in unit field (we use the subscript here to allow for the possibility that both electron and hole excitation occur at the same energy, i.e., $x_{i}$ takes on either one of two possible values). Then each contributes a charge, $\frac{x_{i} V / w}{w} . e$, to the external circuit, where $\mathrm{w}$ is the distance between the electrodes and $\mathrm{V}$ is the applied voltage. Then if $N$ quanta per sec. fall on the crystal, the observed photocurrent will be

$$
i=N \cdot \frac{a}{K} \frac{e V}{w^{2}} \sum_{i} x_{i} p_{i} K_{i}
$$

or

$$
\sum_{i} x_{i} p_{i} K_{i}=\frac{i}{N} \cdot \frac{K}{a} \cdot \frac{w^{2}}{e V}=Y \text {, say. }
$$

$\mathrm{K}$ was determined by optical absorption measurements on each crystal and $\underline{a}$ calculated therefrom. Thus, from a'combination of optical absorption and photoconductivity measurements, the quantity $Y(E)$ could be determined.

In the literature, photoconductivity data is usually presented by plotting either $i / N$ versus $E$ or $i / N a$ versus $E$. The former method ignores the fact that some of the radiation may be lost by transmission through the sample, while the second does not allow for the possibility that the major absorption processes may not contribute to the photoconductivity. That these assumptions may lead to serious error will be shown below.

An expression for a which accounts for the light absorbed on the first traversal through the crystal and also for that absorbed on the second traversal (i.e., after partial reflection 
at the second Mgo-air interface) may easily be shown to be

$$
a=(1-R)\left(1-e^{-K d}\right)\left(1+R e^{-K d}\right)
$$

where $R(E)$ is the reflection coefficient of $\mathrm{MgO}$ and $\mathrm{d}$ is the crystal thickness. Then the correction factor $\mathrm{K} / \mathrm{a}$ in equation (1) is

$$
\frac{K}{a}=\frac{K}{(1-R)\left(1-e^{-K d}\right)\left(1+R e^{-K d}\right)}
$$

Now for Kd sufficiently small (say<0.1), a good approximation to (3) if $R \approx 0.1$, is

$$
\frac{K}{a}=\frac{1}{d}
$$

Hence, this correction factor will have no influence on the spectral dependence of $\mathrm{Y}$ in that region of the spectrum where the absorption coefficient of the crystal is small enough to satisfy the inequality,

$$
\mathrm{Kd}<0.1
$$

On the other hand, for $\mathrm{Kd}$ sufficiently large (say $>2.5$ ) a good approximation to (3) is

$$
\frac{\mathrm{K}}{\mathrm{a}}=\frac{\mathrm{K}}{\mathrm{I}-\mathrm{R}}
$$

In this case, the correction factor is of the utmost importance in the determination of the spectral dependence of $Y$. The neglect of this factor is equivalent to the assumption that the photoconductivity is due to those centers which are responsible for most of the optical absorption. This is not always a justifiable assumption and it may lead to erroneous results. 
All the photoconductivity yields quoted here were calculated according to equations (1), and (3), (4) or (5).

B.3 Dependence of Photocurrent on Light Intensity and Electric Field

In untreated crystals the photocurrents were found to be proportional to the electric field up to about 6,000 volts $/ \mathrm{cm}$. In this range of fields, the dark current was negligible, i.e., less than $10^{-15}$ amperes. However, for fields greater than some critical value (ca. 6,000 volts/cm.), the dark current rose sharply to $10^{-14}$ amperes or more. This current was sufficiently unstable that photoconductivity measurements were impossible.

Usually, the photocurrents were small enough at full light intensity to prevent an investigation of the dependence of current on intensity. However, because of the larger currents available it was possible to perform the:experiment on an X-rayed crystal, using $2.3 \mathrm{ev}$ quanta. In this case, the photocurrent was proportional to intensity for a 40 to 1 variation of intensity.

Because of the irregular spectral output of both the carbon arc and the mercury arc, it is believed that any significant deviations from proportionality in the cases of other types of crystals would have led to corresponding irregularities in the calculated yield curves. Since the latter irregularities were not observed, we conclude that in the range of intensities used (at least 1,000 to 1 ) the photocurrent was proportional to light intensity.

B.4 Evidence that Photocurrents are a Bulk Effect

In view of the fact that the observed currents were so small, the possibility that they were due to a surface effect 
must be considered. To investigate this possibility the photoconductivity of a thick crystal was measured. A section was cleaved off and the remainder remeasured. This procedure was repeated several times, measuring the photoconductivity and absorption at each step. Table $I$ shows $Y$ and $K$ for the different sections of the same original crystal.

\section{Table I}

Absorption and Photoconductivity at $5.0 \mathrm{ev}$

Thickness $(\mathrm{cm}) \quad i / N \frac{\text { electrons }}{\text { quanta }} \times 10^{10} \mathrm{Y} \times 10^{10} \frac{\mathrm{cm}}{\text { volt }} \mathrm{K}\left(\mathrm{cm}^{-1}\right)$

$\begin{array}{llll}.814 & 7.1 & 1.85 & 1.92 \\ .498 & 4.5 & 1.61 & 2.27 \\ .346 & 5.5 & 2.18 & 1.72 \\ .211 & 3.3 & 1.83 & 1.30 \\ .133 & 2.3 & 1.99 & 1.85 \\ .101 & 1.3 & 1.45 & 2.03\end{array}$

As discussed in Section C.4 the approximate constancy of the third column relative to the second, implies that the measured photocurrents are the result of optical excitation in the bulk of the crystal.

B.5 Space Charge Formation

As explained previously, the exposure of the crystal to light was held to a minimum in order to prevent the formation of space charge fields. In order to demonstrate the development of such fields, the following experiment was performed. With an electric field applied to a crystal, a portion 
of the volume between its electrodes was irradiated with 4.4 ev quanta and the photocurrent measured as a function of time. At intervals the electric field was removed, the electrodes were brought to the same potential by connecting them through the input resistor, and the photocurrent measured under this condition. Figure $3(a)$, curve $A$ shows the variation, with time of irradiation, of the photocurrent with applied field, while curve $B$ gives the current when measured with no applied field. The photocurrents of curve $B$ flow in the opposite direction to those in curve $A$.

The irradiation was then continued with no applied electric field (electrodes connected) and the photocurrent measured as a function of time. At intervals the electric field was applied and the photocurrent measured under this condition. The corresponding curves are presented in Figure $3(b)$. In each case curve $C$ is the sum of curves $A$ and $B$. It is evident from the curves that the flow of photocurrent in the applied field produces space charge fields which persist after the initial exposure to light and after the removal of the potential difference between the electrodes. These results are discussed in a more quantitative fashion in Section C. 5.

B.6 Properties of Crystals as Received

In view of the reasonable agreement among the results from different crystals (Table II) little attempt to correlate optical absorption or photoconductivity data with impurity content has 
been made. Spectrographic analyses of MgO crystals obtained from the Norton Company have, however, been made in this laboratory ${ }^{33}$. These analyses show that the principal impurity is iron $(.01-.05 \%)$ with somewhat smaller concentrations of manganese, chromium, calcium and silicon.

As received from the Norton Company, most of the MgO crystals employed in this investigation showed an optical absorption spectrum as shown in Figure 4, curve A. The exceptions to this rule had a spectrum as shown by curve $B$. The actual magnitude of the absorption varied considerably from crystal to crystal but these two shapes were nearly always found.

The photoconductivity spectra of crystals characterized by absorption spectra of either type A or type B (Figure 4) were as shown in Figure 5, which gives two typical photoconductive yield curves along with the corresponding absorption curves. All spectra obtained on untreated crystals were of one or the other of these two types, which differ in shape principally in the energy region above $5.0 \mathrm{ev}$. The latter difference coild not be correlated with any known difference among crystals. The photoconductivity in the region below $5.0 \mathrm{ev}$ could be well accounted for in most cases by a superposition of two Gaussian bands centered about $4.05 \mathrm{ev}$ and $5.05 \mathrm{ev}$. The decomposition of a typical spectrum into these two bands is show in Figure 6. The locations of these peaks do not correspond to the positions of any of the previously known optical absorption bands in MgO. The reproducibility of the magnitude of these bands from crystal to crystal is illustrated in Table II, where the yields at 3.8 and $4.6 \mathrm{ev}$ and their ratio are presented for a number 
of specimens. These energies were chosen as being representative of the magnitudes of the low and high energy peaks, respectively. Considering that the measured yield depends both on the density of photoionizable centers and on the range of the free carriers, both of which may depend upon impurity concentration, it is perhaps surprising that the magnitude of the yield does not show a greater variation from crystal to crystal. The magnitude variations which did occur could not be correlated with variations of magnitude nor type in the optical absorption spectrum. 


\section{Table II}

$\frac{\text { Photoconductive Yield of a Number of Specimens }}{\text { Type of }}$

\begin{tabular}{|c|c|c|c|c|}
\hline $\mathrm{Y}_{3.8} \times 10^{13}$ & $\begin{array}{l}Y_{4.6} \times 10^{11} \\
(\mathrm{~cm} / \text { volt })\end{array}$ & $\mathrm{Y}_{4.6} / \mathrm{Y}_{3.8}$ & $\begin{array}{l}\text { Type of } \\
\text { Absorption Spectrum } \\
\text { (See Fig. 4) } \\
\end{array}$ & Comments \\
\hline 43 & 14.8 & 34 & B & \\
\hline 11.5 & 6.0 & 52 & B & * \\
\hline 4.1 & 2.53 & 62 & & \\
\hline \multirow{2}{*}{14.0} & 7.12 & 51 & B & * \\
\hline & 3.81 & & B & * \\
\hline 12.4 & 6.0 & 48 & B & * \\
\hline 0.9 & 5.74 & 640 & A & \\
\hline 6.2 & 4.89 & 79 & A & Lower Purity \\
\hline \multirow[t]{2}{*}{70.0} & 17.7 & 25 & B & \\
\hline & 4.40 & & B & * \\
\hline \multirow[t]{2}{*}{13.5} & 6.34 & 47 & B & \\
\hline & 5.45 & & $A$ & \\
\hline 6.3 & 3.4 & 54 & A & \\
\hline 14.4 & 4.08 & 28 & A & \\
\hline 8.5 & 4.6 & 54 & B & \\
\hline 6.6 & 4.52 & 68 & B & \\
\hline 9.1 & 5.0 & 55 & $\mathrm{~B}$ & * \\
\hline
\end{tabular}

NOTES: 1. Samples marked* were cut from the same larger piece.

2. The sample designated "lower purity" was impure enough to be visibly colored. The principal impurity was probably iron.

The photoconductive yield at $4.8 \mathrm{ev}$ (i.e. in the $5 \mathrm{ev}$ band) was found to be approximately twice as great at $250^{\circ} \mathrm{C}$ as at room 
temperature. With the experimental arrangement employed the temperature variation of the $4 \mathrm{ev}$ band could not be measured.

\section{B.7 Heat Treatment Studies}

As has been previously mentioned (Section B.6 and Fig. 4A) most of the crystals as obtained, possessed an ultraviolet absorption spectrum consisting of two obvious bands. For crystals of normal thickness $(0.2$ to $0.7 \mathrm{~mm})$ the two absorption bands could be removed by heating in vacuum at $1400^{\circ} \mathrm{C}$ for several hours. Such a treatment resulted in spectra like that shown in Figure 4 , curve $B$. Further heating at the same temperature did not change the shape of the spectrum. For convenience we will refer to crystals with this type of absorption spectrum as "stoichilometric" although, admittedly, we cannot be sure that this spectrum is not characteristic of a small deviation from stoichiometry, in equilibrium at the high temperature. The spectrum itself will be termed the "background" absorption.

The effect of heat treatment at various temperatures on the absorption of stoichiometric crystals was studied in some detail. Since no conclusions could be drawn from the detailed behavior of the absorption at the various temperatures, only the most important observations will be given here.

Figure 7, curve $C$ shows the spectrum of a specimen which was cooled slowly from $1400^{\circ} \mathrm{C}$. This type of spectrum was also characteristic of specimens heated at $1000^{\circ} \mathrm{C}$ or $1100^{\circ} \mathrm{C}$ and quenched therefrom. The following observations summarize the experiments performed at $1000^{\circ} \mathrm{C}$ and $1100^{\circ} \mathrm{C}$.

1. The change of spectral shape from that of curve $A$ to 
that of curve C (Fig. 7): was reversible. That is, the curve A could be regained by reheating at $1400^{\circ} \mathrm{C}$ and quenching from this temperature.

2. The rate at which the equilibrium shape $C$ (Fig. 7) was approached was essentially the same at $1000^{\circ} \mathrm{C}$ as it was at $1100^{\circ} \mathrm{C}$. At both temperatures this rate was found to be independent of crystal thickness.

3. The change in shape of the optical absorption curve was not accompanied by a change in the spectral dependence of the photoconductivity. This point is illustrated by Fig. 8 which shows the yield curves for two crystals, one heated at $1400^{\circ} \mathrm{C}$ for 2 hours, the other at $1000^{\circ} \mathrm{C}$ for 230 hours. The absorption spectra were of the types $A$ and $C$ (Fig. 7) respectively.

\section{B. 8 Excess Mg Crystals}

A typical absorption spectrum for a crystal heated in Mg vapor is shown in Figure 9, curve B. Since the shape of the curve differed somewhat for different coloring conditions, two experiments designed to determine the cause of this change of shape were carried out, viz.,

1. A crystal $.068 \mathrm{~cm}$ thick was heated for 1 hour at $1115^{\circ} \mathrm{C}$ in a $\mathrm{Mg}$ pressure of approximately 2 atmospheres. The spectra before and after this treatment are shown in Figure 10. Figure 11 shows similar curves for a crystal $.074 \mathrm{~cm}$ thick treated in a similar fashion for 3 hours. Both of the se crystals were lightly colored compared to that shown in Figure 9.

2. A crystal $.077 \mathrm{~cm}$ thick was heated 4 hours at $1115^{\circ} \mathrm{C}$ 
in about 2 atmospheres pressure of $\mathrm{Mg}$. The absorption change is shown by curve $A$ of Figure 12. A further similar treatment of 5 hours was carried out. Curve B shows the extra absorption induced by the second treatment only.

The above two experiments show that the true induced absorption could not be calculated by simply subtracting the absorption measured before heating in $\mathrm{Mg}$ vapor from that measured after heating. This point is discussed more thoroughly in Section C.8. The photoconductivity of the more strongly colored crystal of Figure 9 is shown in Figure 13, curve B. This specimen had been treated for 48 hours at $1200^{\circ} \mathrm{C}$ in a Mg pressure of $1 \mathrm{~mm} \mathrm{Hg}$. For comparison, the spectrum of a typical stoichiometric or excess oxygen crystal is also included (curve A).

\section{B.9 The Effect of Ultraviolet Irradiation}

The effect of ultraviolet irradiation on the photoconductivity of stoichiometric crystals was studied by irradiating a crystal with low energy quanta, measuring the yield at five different energies, irradiating at a higher energy, remeasuring, etc. Figure 14 shows the results of this experiment. The numbers along the horizontal axis indicate the energy of the quanta leading to the photoconductivity spectrum with the corresponding number.

In view of the apparent presence of two absorption bands which lead to appreciable photoconductivity (Figure 6), the ultraviolet irradiation experiments we re repeated on two other crystals one of which was irradiated at $3.8 \mathrm{ev},\left(1.6 \times 10^{19}\right.$ quanta $\left./ \mathrm{cm}^{2}\right)$ the other at $4.6 \mathrm{ev}\left(4 \times 10^{17}\right.$ quanta $\left./ \mathrm{cm}^{2}\right)$. These 
energies were chosen so that the activation would in each case be due to absorption in only one of the two bands. The results of these experiments are shown in Figure 15, which also shows for comparison a typical spectrum before irradiation.

The absorption changes produced by such irradiations are shown in Figures 16 and 17.

\section{B.10 The Effect of X-Irradiation}

The effect of $\mathrm{X}$-irradiation on the optical absorption and photoconductivity was studied by exposing stoichiometric crystals to the beam from an X-ray tube with a tungsten target and beryllium window. The samples were placed about $8 \mathrm{~cm}$ from the target and the tube was operated at $50 \mathrm{kv}$ and $15 \mathrm{ma}$. During the irradiation the specimens were covered with aluminum foil to protect them from optical radiation.

Figure 18 shows the induced absorption at two quantum energies as a function of the irradiation time. Similar curves were obtained for all quantum energies so that to a good approximation it may be said that the shape of the induced absorption spectrum does not vary during the period of $\mathrm{X}$-ray irradiation. The saturated induced absorption spectrum at room temperature is shown in Figure 19, curve $A$.

The specimen was stored in the dark at room temperature and the absorption measured at intervals. Figure 19, curve B, shows the induced absorption after 95 hours of dark decay while the absorption at two quantum energies is plotted as a function of time in Figure 20.

Figure 21 shows the effect of X-rays on the photoconductivity. 
Curve $A$ corresponds to the untreated crystal. The $5.0 \mathrm{ev}$ band is obvious but the $4.0 \mathrm{ev}$ band is just resolved in this particular case. The crystal was exposed to $X$-rays for 40 minutes and curve $B$ measured immediately after this irradiation while curve $C$ was measured 4 hours later. After 50 hours the yield had changed to curve $D$ and changed only very slowly thereafter.

Figure 22 shows similar data for the same crystal in the low energy region of the spectrum. Curve $A$ was obtained immediately after irradiation; curve $B, 72$ hours later. The change in absorption coefficient during the same period is shown by the dotted curves in the same figure.

By comparing Figure 15, curve $C$, with Figure 21, curve $D$, it may be seen that the photoconductivity of a specimen activated by irradiation in the 5.0 ev band is similar to the photoconductivity of specimens irradiated with $\mathrm{X}$-rays and allowed to decay thermally. The effect of subsequent $\mathrm{X}$-irradiation on a previously UV-irradiated crystal is shown in Figure 23. Curve $A$ was obtained by irradiation with 4.4 ev quanta, i.e., the irradiation was in the $5 \mathrm{ev}$ band and the spectrum is therefore similar to that of Figure 18, curve C. Curve B resulted from the $X$-ray exposure, while curve $C$ was obtained after 96 hours of subsequent thermal decay.

The extra absorption and photoconductivity induced by $\mathrm{X}$-irradiation can be reduced by optical irradiation. A thorough study of this effect has not been made but the effect of $2.3 \mathrm{ev}$ quanta has been studied to some extent. Figure 24, curve $A$, shows the variation with time of the 
optical absorption at $4.2 \mathrm{ev}$, when a freshly X-rayed crystal was irradiated with $3 \times 10^{15}$ quanta/ $\mathrm{cm}^{2}$-sec. Curve $B$ is for a control sample $\mathrm{X}$-rayed at the same time but stored in the dark at room temperature. Similar curves for the absorption at $2.3 \mathrm{ev}$ are shown in Figure 25. The absorption at all other quantum energies was likewise reduced by the irradiation.

The variation with integrated light flux, of the photoconductive yield of a partially decayed, X-rayed crystal, is shown in Figure 26. In this case the optical absorption was not measured during nor after the irradiation. The data on Figure 26 was plotted assuming that the factor $\mathrm{K} / \mathrm{a}$ in equation (I) remained constant during the irradiation. Since we know from Figures 24 and 25 that this factor actually did decrease during irradiation (especially for the high energies), it may be seen that the left hand sides of the curves in Figure 26 should be raised relative to the right hand sides. Since the amount of this increase in largest for the energies $3.5,4.0$ and $4.5 \mathrm{ev}$, it would tend to make the shapes of all curves more nearly the same.

Since the optical absorption at the beginning of the irradiation was known, the true yield ( $Y$ ) at this time could be calculated. Then, by assuming that the irradiation reduced the absorption to a small value, K/a after the irradiation was calculated from equation (4). Since this assumption gives a lower limit to $\mathrm{K} / \mathrm{a}$, the total fractional change in yield calculated therefrom will be an upper limit. Thus the true values of total fractional change in yield lie between those in the two columns of the table following. 
Table III

Energy

(e.v.)
Total Fractional Changes in Yield (\%) Uncorrected

Over-Corrected
2.3

3.0

3.5

4.0

4.5
61

61

57

56

53
61

63

60

65

70

The significant point illustrated by Table III is that the reduction in photoconductive yield is essentially independent of energy. As discussed in section C.10 this implies that the main effect of $2.3 \mathrm{ev}$ irradiation is to empty some levels which have been filled during this $\mathrm{X}$-ray excitation, thus reducing the electronic range.

\section{B.11 Sign of the Charge Carriers}

In order to determine the sign of the optically induced current carriers, the field distribution due to the photoelectrically produced space charge regions (see section C.5) was studied. For each spectral region of interest a central region (such as $\underline{b}$ in Figure $3(\mathrm{c}$ ) of a crystal was irradiated with the electric field applied for periods ranging from 20 minutes to several hours. When the photocurrent was reduced to a low value by the formation of the space charge field, the applied voltage was removed, the two electrodes of the crystal were connected together through the amplifier input resistor (see Figure 1) and a narrow beam of light of the 
same quantum energy was moved across the crystal by means of the micrometer L (Figure 1). In this way the photocurrent could be measured as a function of the position of the light beam on the crystal. If the photoconductive sensitivity of the crystal were unaffected by the irradiation, the plot of photocurrent versus position would also be a plot of space charge field versus position. The effects of irradiationinduced changes in photosensitivity could be minimized in a number of ways and therefore approximate space charge field distributions could be obtained.

To determine the sign of the charge carriers produced by quanta in the $4.0 \mathrm{ev}$ band, the following experiment was performed: An untreated stoichiometric specimen was irradiated over its whole volume for a period of 42 hours, with no applied electric field; 3.9 ev quanta were used for this irradiation to ensure that little absorption occurred in the $5.0 \mathrm{ev}$ band. The spectral distribution of photoconductivity after this irradiation has already been given in Figure 15, (curve B). An electric field was then applied to the crystal and the central region irradiated with $3.8 \mathrm{ev}$ quanta to create a space charge field. The electrodes were then brought to the same potential and the field distribution was determined as described above, using a light beam $1 / 3$ of the width of that used in the irradiation. This field distribution is shown in Figure 27, curve $A$. The irradiation was then continued, using the wider beam, until the photocurrent was again reduced to a small value. The resulting field distribution is shown by curve $B$ of Figure 27 . The most 
significant feature of the latter curve is the shift of the field minimum towards the negative electrode with respect to the minimum of curve $A$. This was a reproducible feature of the experiment.

A similar experiment was performed on a different crystal this time using $4.6 \mathrm{ev}$ quanta i.e. irradiating in the $5.0 \mathrm{ev}$ absorption band. In this case, however, the sample was not given an initial overall irradiation to homogenize the sensitivity. For this reason the photocurrent distributions obtained represented electric field distributions only when the scanning beam was within the limits of the irradiated region. The distributions obtained are shown in Figure 28, curve $A$ after one irradiation with electric field applied, curve $B$, after a subsequent irradiation with no applied field, and curve $C$ after a further irradiation also without field. ("No applied field" always implies that the electrodes were maintained at the same potential.)

The sign of the charge carrier was also determined for the $\mathrm{X}$-rayed crystal corresponding to curve D of Figure 21. The field distributions are shown in Figure 29. In this case $4.4 \mathrm{ev}$ quanta were used to form and to detect the space charge. In this experiment the most significant feature is the appearance in curve $B$ of a minimum displaced toward the positive electrode from another minimum corresponding in position to the minimum in curve $A$.

The interpretation of these experiments is presented in section C.ll. 


\section{DISCUSSION}

\section{C.1 Optical Absorption}

The usual model used in discussing the properties of solids involves the introduction of a periodic potential due to all nuclei and all the electrons but one. The eigenvalues and eigenfunctions of the remaining electron are then given by the solutions of the Schrodinger equation with this potential. The most important feature of this model is the occurrence of quasicontinuous groups of energy eigenvalues spearated by energy regions which are forbidden.

The disruption of the periodic potential by some type of imperfection results in the occurrence of localized energy levels in the normally forbidden region. It is the excitation of electrons to or from such levels in $\mathrm{MgO}$ that is the main concern of this thesis.

Since the region of the crystal in which the deviation from periodicity occurs (hereafter referred to as the "center") will usually have associated with it more than one energy level in the normally forbidden band ${ }^{29}$, we may expect that transitions between these levels may be of some importance. Thus one may expect to find associated with the centers certain absorption bands. There are many examples of this type of absorption in the literature ${ }^{30}$.

The fact that bands occur rather than lines, (as might be expected for transitions between discrete states) has been well explained as due to interaction between the localized electron and the thermal vibrations of the lattice ${ }^{31}$. 
Calculations of the variation of absorption with quantum energy have been made on a similar basis. It is found that under most circumstances the absorption bands can be well approximated by Gaussian curves.

Optical transitions which take electrons between localized levels and energy bands of the perfect crystal are of course also possible. Numerous examples of this type of absorption have also been reported ${ }^{32}$. The absorption in these cases has usually been detected by photoconductivity measurements. Since the final state of the transition is not discrete the absorption may be expected to extend over a wider range of energy than in the previous case and in addition will not be symmetrical about some central energy.

\section{C.2 Discussion of the Factors Involved in the Photoconductive Yield}

In the analysis of the photoconductivity data described in section B.2, a factor $\mathrm{p}$ was introduced to represent the probability that, following an electronic excitation, a free charge carrier be formed. Such a factor is necessary in order to take account of the possibility that the excitation may take place, not to an energy band but rather to another discrete level belonging to the same absorption center. In this case, the formation of a free charge carrier requires a subsequent process by which the particle in the excited state may gain sufficiently more energy that it may be transferred to an energy band. The extra energy may be obtained by the absorption 
of lattice phonons, for example. Under these conditions the photoconductivity may be expected to contain a strong temperature dependence in a certain temperature interval. A good example of such behavior is given by $\mathrm{KCl}$ crystals containing a stoichiometric excess of potassium ${ }^{4}$.

In contrast to the weak temperature dependence mentioned in section B.6, Day ${ }^{5}$ has reported that the photoconductivity in MgO decreased $10^{5}$ times on lowering the temperature to $90^{\circ} \mathrm{K}$. Although we cannot determine definitely from Day's paper whether this result applies to the untreated crystal (as does the present result) it is believed that it applies to neutron irradiated samples so that there may be not disparity between the two results.

The quantity $x$ introduced in equation (1) was defined as the mean distance a charge carrier moves during its lifetime, when the electric field is unity. This quantity can be writtèn

$$
\mathrm{x}=\mu \mathrm{T}
$$

where $\mu$ is the mobility of the carriers and $T$ their mean lifetime. The latter, of course, is determined by the density and capture cross-section of levels in which the carriers may be trapped. This trapping may be temporary or permanent, i.e., thermal release from the trap may occur within the time of a measurement or only after a time which is long compared to the latter. In the former case, the photocurrent will be observed to increase during the time of the measurement. Since no such "secondary" effects were 
noted in these experiments, the trapping in MgO may be regarded as permanent. Indeed it is concluded in a later section that some electrons may be trapped for many months.

In equation $(1)$ we have employed the fact that a charge e displaced a distance $x$ in the electric field will be observed externally as the passage of a charge $\frac{e x}{w}$. This fact follows from simple electrostatics but the derivation will be deferred until section C.5 where the appropriate equations are developed for another purpose.

The equation for the photoconductive yield developed in section B.2 gives for a given crystal, constant electric field, and spectral region of strong absorption (equation (5), the proportionality

$$
\mathrm{Y} \sim \frac{i}{\mathrm{~N}} \mathrm{~K} .
$$

Thus if the ratio of photocurrent to incident light flux $(i / N)$ is plotted against quantum energy a distorted picture of the true absorption. spectrum of the photoionized centers will be obtained since

$$
\frac{i}{N} \sim \frac{Y}{K}
$$

It can easily be seen that in spectral regions where peaks in the total absorption $(K)$ exist and where the photoionization varies relatively slowly with energy, spurious minima in $i / N$ may be obtained. It is believed that the disagreement between the present results and those of Day ${ }^{5}$ are completely due to this cause. A more detailed comparison of 
the two sets of results will be given in a later section.

\section{C.3 The Dependence of the Photocurrent on Light Intensity and Electric Field}

The threshold electric field above which a large increase in dark current occurred (see section B.3) was not observed by Day $^{5}$, although filelds up to 14,000 volts/cm we re employed. In the present work the cirtical field was about 6,000 volts $/ \mathrm{cm}$. The reason for this discrepancy is not known although two possibilities have been considered. In the first place, the electrodes used in Day's work and the present work were Aquadag (colloidal graphite applied in water suspension) and silver paste, respectively. It is difficult to define a work function for such materials but it is likely that the "effective work function" for strong field emission 6 is different for the two cases. If the sudden onset of a large dark current were due to strong field electron emission from the negative electrode, the threshold field would be expected to be different in the two cases.

Secondly, although in the work of Day the crystals were measured in dry air, in most of the present measurements it was not found necessary to desiccate the sample chamber during the measurement of the photoconductivity spectra. Consequently, a surface breakdown might occur at a smaller electric field due to a surface film of e.g. hydroxide.

In any case, whatever the cause of the high dark currents they could be readily avoided simply by maintaining an electric field less than the critical value. The small dark 
current which did flow under the latter circumstances did not cause measurable space charge development.

The experimental observation of the linear rellation between photocurrent and electric field strength is a justification for equation (1) and the assumption implicit therein, viz. that none of the excited carriers reach the electrodes, for if they did, a tendency for the photocurrent to saturate with increasing field would have been observed. Thus, knowing the distance from the edge of the irradiation region to the electrode, an upper limit on $\mathrm{x}$ may be obtained. This limit is

$$
x<10^{-5} \mathrm{~cm}^{2} / \text { volt }
$$

for untreated crystals. Similar experiments have not been performed on X-rayed or UV irradiated specimens.

As mentioned above, $\mathrm{x}$ is determined by the lifetime of the excited carriers. A carrier may end its life by one or other of the following mechanisms: (see Fig. 30)

(a) Direct recombination with a free carrier of the opposite type,

(b) recombination with a free carrier of the opposite type through some imperfection-contributed intermediate state,

(c) return to a level of the same type as that from which it came or,

(d) trapping by a level other than that from which it came.

Process (a) (Figure $30(a)$ is of no importance when excitation occurs in a single absorption band, assuming of course, 
that the quanta are not sufficiently energetic to cause bandto-band transitions and thus create electron-hole pairs. Since the forbidden energy region is $10.5 \mathrm{ev}$ wide 15,16 this condition is met in all the present measurements. In the energy region of band overlap (Figure 6), however, it is conceivable that both electrons and holes may be simultaneously excited and direct recombination must be considered. Such recombination, however, leads to a non-linear relationship between photocurrent and light intensity ${ }^{7}$. Since we have concludedilsection B.2) that, for the range of intensities used and the spectral region investigated, the photocurrent was proportional to light intensity, we conclude also that direct recombination is not a dominant mechanism.

Recombination through intermediate states (Figure 30(b) would again be important only in a spectral region where both free electrons and free holes were being generated. For the low excitations obtained in these experiments, a linear dependence of photocurrent on light intensity would be expected in this case ${ }^{8}$. Thus mechanism (b) cannot be ruled out by the same argument used to eliminate (a).

However, if this mechanism were important one would expect to find an abnormal decrease in photoconductive response as the quantum energy reached the spectral region of absorption band overlap. It may be seen from Figure 6 that such a decrease was not observed in untreated or vacuum heated crystals. Therefore, it is believed that free hole-electron recombination is not an important mechanism in such crystals. 
Since UV or X-ray excitation can hardly be expected to change the number or type of levels through which the recombination can occur the same conclusion may be applied to such crystals. The third mechanism listed above was the return of excited carriers to the same type of center from which they came. If this center had previously been emptied by optical excitation, such a trapping event would assure that the net effect on the properties of the crystal would be zero. Since the results of section $B .9$ show that the photoconductive spectrum was altered by prolonged UV irradiation in either of the two bands it must be concluded that this type of trapping event is not the only one which can occur. Also, simple considerations show that this mechanism leads to a non-linear dependence of current on light intensity. For this reason one can make the stronger conclusion that the trapping of carriers in already ionized centers of the type from which they came is not the dominant recombination mechanism. If on the other hand the excited carriers were to be trapped in centers exactly similar (i.e. still occupied) to those from which they came (Figure $30(\mathrm{c})$, a change in spectral response would be expected and a linear dependence on intensity would be obtained.

Trapping by previously unoccupied levels (Figure $30(d)$ would also fulfill the two conditions required by the experimental results and discussed above. The occupancy of such levels comprises a thermodynamically unstable situation which will revert to the original state, given enough time. In view of the fact that space charge distributions which last 
for considerable periods of time can be formed (section B.5) it can be concluded that at least some of the trapped carriers have release times much greater than 0.5 seconds. It is concluded in section C.10 that at least some of the electrons remain trapped for several months.

Thus, the most likely recombination mechanisms seem to be the trapping of excited carriers by centers of the same type as those from which they were excited and/or by other, normally empty, more shallow levels. It is tentatively concluded in section C. 6 that for untreated crystals both mechanisms are operative.

C.4 Evidence that the Photocurrents are Due to a Bulk Effect The question of photocurrents excited in surface films (e.g. magnesium hydroxide) arises because of the low sensitivity of the MgO crystals to optical irradiation. If the currents we re primarily due to surface conduction, the contribution from the front surface (i.e. the side on which the light beam was incident) would remain constant as the thickness of the specimen was reduced. If the surface layer were to form rapidly (in c. 1 hour, say) then the contribution from the back, freshly cleaved, face would be proportional to the light incident on it and would thus increase somewhat as successive pieces of the specimen were cleaved off. Thus the quantity $i / N$ would be expected to increase slightly as the thickness of the specimen was reduced.

On the other hand, if the photosensitive surface layer 
required a very long time to form, only the trial represented by the first line of Table I would be expected to contain a contribution from both surfaces. In this case $i / N$ would decrease by less than a factor 2 after the first reduction in thickness and remain constant thereafter.

If the measured photoconductivity were exclusively a bulk effect, the calculated yield $Y$ would be expected to be constant, independent of thickness.

The quantity ${ }^{i} / N$ did not vary in either of the above discussed fashions (see Table I). The observed variation in $Y$ was slightly greater than the experimental error. However, the non-uniformity of the crystal, as evidenced also by the optical absorption (last column, Table I) may be responsible for this variation. It should be emphasized that such a volume non-uniformity would not affect a surface photosensitivity.

It is believed that the above discussed experiment provides convincing evidence that the major portion of the photocurrent was excited in the volume of the crystal. All further discussion will be presented on this basis.

\section{C.5 Formation of Space Charge Fields}

The results presented in Figure 3 can be explained in, terms of a simple model, which will be discussed by reference to Figure $3(\mathrm{c})$.

The YZ planes of the crystal constitute the electrodes. A portion of the $\mathrm{XZ}$ plane was illuminated with a beam of 
light parallel to the $\mathrm{Y}$ axis. Upon illumination, some excited carriers travel to the edge of the illuminated region and even outside it. If the range of the carriers is small compared to the width of the irradiated region, the net result of the irradiation with field can be considered as the formation of a sheet of charge at either side of the irradiated region. Let the charge in each sheet be \pm ne per $\mathrm{cm}^{2}$. Then if $E(x)$ is the electric field and $\varepsilon$ the dielectric constant, we have from Poisson's equation

$$
\Delta \vec{E}= \pm \frac{4 \pi}{\varepsilon} \text { ne }
$$

where $\Delta \mathrm{E}$ is the discontinuity in the electric field at either side of the irradiated region. We also have

$$
E_{a_{1}} \cdot a_{1}+E_{b} \cdot b+E_{a_{2}} \cdot a_{2}=V
$$

where: $E_{a}$ and $E_{b}$ are the electric fields in their respective regions, $V$ is the potential difference between the electrodes and $d$ is the crystal thickness. From equations (6) and (7) and the definition of $\Delta E$ we obtain

$$
E_{b}=\frac{V}{d}-\frac{4 \pi n e}{\varepsilon} \quad\left(1-\frac{b}{d}\right)
$$

With the electrodes at the same potential

$$
E_{b}^{\prime}=-\frac{4 \pi n e}{\varepsilon}\left(1-\frac{b}{d}\right)
$$

When the condition $E_{b}=-E_{b}^{\prime}$ has been achieved; the photocurrents measured with and without the applied electric field should be equal in magnitude but opposite in sign. The time, 
T, at which this condition held can be obtained from Figure 3(a) and we have from (8) and (9),

$$
n(T)=\frac{V \varepsilon}{8 \pi e(d-b)}
$$

For the experimental conditions employed it was calculated that

$$
n(T)=8.7 \times 10^{9} \text { electrons } / \mathrm{cm}^{2} .
$$

Now the formation of this space charge is equivalent, as far as the external circuit is concerned, to the transfer of a charge $n(T)$ Ae from one side of the illuminated region to the other, a distance $b$ ( $A$ is the cross sectional area of the crystal in the YZ plane).

$$
\int_{0}^{T} i \cdot d t=n(T) \frac{\text { Abe }}{d}
$$

The reason for the factor $\frac{b}{d}$ on the right hand side of Equation 11 is explained later in this section.

By integrating under the experimental curve $A$ of Figure $3(a)$ the left hand side of Equation (II) was determined and $n(T)$ calculated to be

$$
n(T)=9.0 \times 10^{9} \text { electrons } / \mathrm{cm}^{2} \text {. }
$$

Thus the two values of $n(T)$ agree within the experimental error.

The curves of Figure 3 are approximately exponential in character. A simple analysis would show that they should be 
precisely exponential if the UV irradiation had no effect on the crystal (except, of course for the formation of the space charge field). If the latter were the case the absolute values of the photocurrents measured with and without electric field should have a constant sum (see equations 8 and 9). Curves $C$ of Figure $3(a)$ and (b) show that this is not so. From the results of section $B .9$, however, an activation of the crystal was to be expected in this experiment, so that curves $C$ of Figure 3 should increase as a function of time. The maximum in Figure $3(a)$ and the minimum in Figure $3(b)$ are not explained.

It should be emphasized that the presence of this activation effect has no influence on the foregoing calculations of space charge density (equations (10) and (11). Indeed the comparison with experiment was done in the above fashion in order to eliminate the effect of the changing photosensitivity with time of irradiation.

In the work of $\mathrm{Day}^{5}$, it was found that when a narrow region in the center of a crystal was illuminated, the photocurrent decreased initially by a factor two, then remained constant for many hours. The initial decrease may have been due to the development of space charge fields as described above. The fact that the current was not reduced to zero is in agreement with the results obtained, in certain cases, in the present experiments. A possible explanation of this non-reproducible result is as follows: As the electric field in the illuminated region is decreased due to the formation of the space charge, that in the dark region is increased (equation (7). Thus, 
depending on the magnitude of the initial electric field and the relative magnitudes of $\underline{b}$ and $\underline{d}$ (equations (7) and (8), the electric field in the dark region may increase to the critical value (section C.3) even though the initial, uniform, field was well below this value. If this were the case, the equilibrium current observed would consist in part of "dark current" and the question, raised by Day, as to the mechanism allowing the passage of continuous photocurrents would be revolved into the same question concerning the passage of continuous dark currents.

From this experiment it may be concluded that the photoconductivity mechanism may be the most obvious one, viz., the excitation of carriers to an energy band, their motion therein, and their subsequent trapping with consequent formation of space charge layers at the boundaries of the irradiated region.

From the above equations it is easily shown that the motion of a charge between the electrodes is measured by the external circuit as a smaller charge by the ratio of the distance moved to the electrode separation. (This was assumed in equation (1).) To see this we first apply Gauss' Theorem to a rectangular parallelepiped containing the left hand electrode of the crystal (see Figure $3(\mathrm{c}$ ) and having two of its faces parallel to this electrode. Then ignoring the fringing of the electric field (as in the derivation of equations (8) and (9) we obtain

$$
\varepsilon E_{a_{1}:} \quad A=4 \pi q_{1}
$$


where $q_{1}$ is the charge on the electrode. A charge $e$ is now moved from $x=a_{1}$ to $x=a_{1}+b$ where $a_{1}$ and $b$ are now arbitrary. From (12)

$$
\Delta \mathrm{q}_{1}=\frac{\varepsilon A}{4 \pi} \cdot \Delta \mathrm{E}_{\mathrm{a}_{1}}
$$

and of course the passage of $\Delta q_{1}$ is observed externally. Now from (6) and (7)

$$
E_{a_{1}^{3}}=\frac{1}{d}\left[V+\frac{4 \pi n e b}{\varepsilon}\right]
$$

and

$$
\Delta E_{i a_{1}}=\frac{4 \pi n e}{\varepsilon} \cdot \frac{b}{d}
$$

For the case under consideration the charge $/ \mathrm{cm}^{2}$, ne must be replaced by e/A so that (14) becomes

$$
\Delta E^{\cdots} \mathrm{d}_{1}=\frac{4 \pi e}{\varepsilon \mathrm{A}} \cdot \frac{\mathrm{b}}{\mathrm{d}}
$$

and combining (13) and (15)

$$
\Delta q_{1}=\frac{b}{d} \cdot e
$$

which is the desired result.

C.6 Properties of Crystals as Received

The two obvious absorption peaks in spectra such as curve A, Figure 4 were shown by Weber ${ }^{10}$ to be characteristic of the presence of a stoichiometric excess of oxygen in the crystal. "This fact has been verified by Sosheall and in addition he has shown that such spectra also contain a peak 
centered at $4.8 \mathrm{ev}$ which is masked by those at 4.3 and $5.7 \mathrm{ev}$.

None of these three bands were observed in the photoconductivity spectra. Consequently it must be concluded that the probability $p$ of thermal ionization of the excited state is considerably smaller than for the 4.0 and 5.0 bands which were observed in photoconductivity. Since the mean range, $x$, is sufficiently large for conduction by either holes or electrons to be observed (section C.11) it cannot be argued that this is the factor which prevents the observation of the three excess oxygen bands in photoconductivity measurements. Similarly in many of the specimens measured the total absorption was dominated by these bands. Thus as has already been stated, p must be very small for the excess oxygen centers. Since it is reasonable to expect that the excess oxygen is incorporated substitutionally as a divalent ion, the observed optical transitions thus produced may be expected to result from electronic transitions to levels which have been emptied to complete the outer shell of the ionic excess oxygen. Thus, if the transitions took place from the valence band they might be visualized as in Figure $31(a)$. However, it has been argued above that the transition produces a free hole with very small probability. Hence, we visualize transitions such as that illustrated by Figure $31(b)$.

It has been observed by several workers $10,11,12$ that the ratio of the intensities of the 4.3 and $5.7 \mathrm{ev}$ bands is 
strikingly constant under rather widely varying conditions, while the $4.8 \mathrm{ev}$ band has a different intensity relative to the other two, depending on the conditions of formation of the bands. These facts strongly suggest that the 4.3 and $5.7 \mathrm{ev}$ transitions occur in the same center while the $4.8 \mathrm{ev}$ transition may be characteristic of a different center.

The 4.0 and 5.0 ev transitions observed in the photoconductivity measurements had not been observed previously. Recently, however, Lye ${ }^{13}$ has definitely identified the $5.0 \mathrm{ev}$ transition in the absorption spectra of stoichiometric crystals and has also observed a small absorption which may be identifiable with the $4.0 \mathrm{ev}$ transition. The magnitude of the absorption was very nearly the same for all crystals and had the approximate values

$$
\begin{aligned}
& \mathrm{K}_{5.0}=0.7 \mathrm{~cm}^{-1} \\
& \mathrm{~K}_{4.0} .05 \mathrm{~cm}^{-1}
\end{aligned}
$$

These values enable an estimate of the product $\mathrm{xp}$ to be made. For this the data of Figure 6 can be employed. We obtain

$$
\begin{aligned}
& \mathrm{xp}_{5.0}=2 \times 10^{-10} \mathrm{~cm}^{2} / \text { volt } . \\
& \mathrm{xp}_{4.0} \quad 3 \times 10^{-11} \mathrm{~cm}^{2} / \text { volt } .
\end{aligned}
$$

The fact that most of the specimens listed in Table II show values of the photoconductive yield of the same order of magnitude in spite of the fact that they were cut from several 
different samples obtained from two different sources, is a very important one. The implications of this fact will now be discussed.

In section C.2 it was concluded the most likely trapping me chanisms were

(a) Capture of the free carrier by a center of the same type as that which initially provided the carrier (Figure $30(\mathrm{c})$ and,

(b) Capture of the free carrier by previously unoccupied, shallower levels (Figure $30(\mathrm{~d})$.

Consider for the moment that mechanism (a) is the dominant one. We have

$$
K \sim n
$$

where $\mathrm{n}$ is the density of the centers in question. Since, according to (a) above the same centers determine the mean range $x$, we have

$$
\mathrm{x} \sim \frac{1}{\mathrm{n}}
$$

Therefore the yield

$$
Y=x p K \sim p
$$

and is independent of the density of the centers.

If, on the other hand, mechanism (b) were to dominate, $\mathrm{K}$ and $\mathrm{x}$ would be independently controlled by the density of absorbing centers and the densities of the shallower levels, respectively. Thus the postulate of a constant number of absorbing centers independent of the crystal source would not 
suffice to explain the reproducible photoconductive yield. Since the postulate of reproducible densities of both absorbing centers and shallow levels ("trapping centers") seems somewhat unlikely, we are led to the hypothesis that the main trapping mechanism is (a). That (b) also occurs is manifested by some variation in the yield from crystal to crystal, and also by the results of the UV activation experiments discussed in section C.9.

Further discussion as to the nature of the 4 and $5 \mathrm{ev}$ centers will be deferred until the sign of the charge carrier has been deduced from the experiments of section B.11. Lower limits on the values of $p$ can, however, be made, using equation (17) and the limit $x<10^{-5} \mathrm{~cm}^{2} /$ volt given in section C. 3 .

We obtain

$$
\begin{aligned}
& p_{5.0}>2 \times 10^{-5} \\
& p_{4.0}>3 \times 10^{-6}
\end{aligned}
$$

\section{C.7 Heat Treatment Studies}

Optical absorption occurring near the fundamental edge in alkali halide crystals is known ${ }^{14}$ to be a structure sensitive property. The fundamental absorption edge itself is believed to be due to electronic transitions from negative ions to neighboring positive ions, in such a way that the electron and its corresponding "hole" on the negative ion remain in interaction. Thus the energy for this transition is lower 
than that for complete removal of the electron. Since all the ions of the lattice can contribute to this absorption, the absorption coefficient is very high $\left(10^{5}-10^{6} \mathrm{~cm}^{-1}\right)$. However, at energies slightly less than that required for this "exciton" transition, the structure sensitive absorption referred to above is always found. It has been suggested that such absorption is due to exciton transitions in ions situated near crystalline imperfections such that a smaller energy is required to transfer the electron. Experimentally it has been shown 34 that the magnitude of the absorption is increased by plastic deformation which is believed to introduce dislocations and vacancies. Thus a study of the optical absorption near the fundamental edge may be considered useful in any investigation of imperfections in ionic crystals. The first fundamental absorption edge in MgO was located at about $7.5 \mathrm{ev}$ by Johnson 15 and was later ascribed to exciton formation by Krumansl ${ }^{16}$. In stoichiometric crystals a measurable absorption occurs, however, at energies as low as $4 \mathrm{ev}$ (Figure 7, curve A) i.e., at roughly one-half the exciton energy. Thus the ultraviolet absorption begins much further from the fundamental edge than it does in typical alkali halide crystals. It can then be argued that either,

(a) The ultraviolet absorption in stoichiometric Mgo is not due to "perturbed" exciton transitions but rather to absorption by impurity atoms, or,

(b) The absorption is due to perturbed exciton transitions and crystalline imperfections have a much greater 
influence on the energy of the exciton transition in MgO than in alkali halide crystals.

It does not seem possible to distinguidis: between the se two possibilities on the basis of the available experimental evidence.

A comparison of curves $A$ and $B$, Figure 7 seems to indicate that the magnitude of the background absorption was decreased by cooling slowly and thus allowing the crystal to approach equilibrium at a temperature lower than $1400^{\circ} \mathrm{C}$, the temperature of the treatment. Curve $C$ however implied that the shape of the absorption spectrum changes with temperature and therefore implies that curve $B$ was merely a nonequilibrium state between the states represented by $A$ and $C$. This implication was further brought out by the subsequent experiments, the details of which were omitted from section B. 7 .

Such a change in the absorption spectrum indicates the formation of new types of imperfections. The fact that more of the latter were formed at lower temperatures suggests that the new imperfections may be formed from the old, e.g. by aggregation. The lack of influence of the crystal thickness on the rate at which the new state is achieved is in accord with this suggestion.

Since the spectral dependence of photoconductivity did not change with heat treatment it must be concluded that the background absorption makes a negligible contribution to the photoconductivity. For this reason we have represented 
(Figure $31(d)$ the transitions involved in such a way that the absorption process will lead to no free carriers.

\section{8 Excess Mg Crystals}

The experiments described in section $B .8$ and illustrated by Figures 10, 11 and 12 show that the optical absorption characteristic of the stoichiometric crystal is reduced by the addition of excess Mg. The actual form of the induced optical absorption can therefore only be deduced from an experiment like that illustrated by Figure 12; assuming that the absorption decrease is essentially complete after the first treatment, the change on the second treatment, curve $B$, gives the actual spectral dependence of the optical absorption. Thus in Figure 9, for example, the total absorption spectrum after the heating in Mg vapor is approximately the induced absorption spectrum.

It is then clear from Figure 9 that the induced spectrum contains no obvious stracture and cannot be resolved into "bands". For this reason we postulate that the spectrum consists of a superposition of several bands; a sufficient number, in fact, that the composite absorption curve shows none of the extrema nor double inflections, which would be expected for the superposition of bands centered about energies sufficiently different from one another.

The most obvious manner in which the excess metal can be accomodated into the lattic is substitutionally as doubly charged positive ions. Thus the extra absorption may be ascribed to the filling of certain unoccupied levels by the 
extra electrons. Such a set of levels is shown in Figure 31(d). Since the filling of these levels reduced the background absorption as discussed above, we associate a filled ground state with each of them. The reduction of the background absorption then follows if transitions such as that illustrated in Figure $34(d)$ are supposed to account for this absorption, as mentioned in the previous section.

It might be concluded from the above that when the background absorption has essentially disappeared (i.e.. the empty levels have been filled) the total absorption will not change further. Experimentally, however, the absorption continues to increase after the initial decrease. It is therefore necessary to assume that the process of adding excess Mg also provides the levels in which the extra electrons are to be assommodated. This may be accomplished, for example, by the diffusion of negative ion vacancies into the body of the crystal.

If the photoconductivity (Figure 13) is to be ascribed to the centers responsible for the bulk of the optical absorption (Figgure 9), it can be concluded that the low energy absorption contributes very little to the photoconductivity. This low energy absorption then cannot be identified with that discussed in connection with $\mathrm{X}$-rayed crystals (section C.10). The latter absorption contributed a measurable photoconductivity. to

The major fraction of the optical absorption may, however, contribute nothing to the photoconductivity. In this 
case two sets of levels such as that shown in Figure 31 (d) would have to be postulated. One of these would be required to account for the absorption spectrum as discussed above while the other would account for the structureless photoconductivity spect rum.

None of the evidence so far presented allows one to choose between the above two alternatives. At this point we arbitrarily select the first possibility since it requires the introduction of fewer levels. As mentioned above, the low energy absorption then must be ascribed to transitions which do not result in free carriers. No attempt has been made to indicate such transitions on Fgiure 3l(d). It is believed that the transitions giving the photoconductivity (and according to our postulate the absorption) at higher energies may be identified with transitions observed in crystals treated in other ways. These are discussed in section C.9 and C.10.

\section{C.9 Ultraviolet Irradiation}

Irradiation with ultraviolet light of an appropriate energy may be expected to empty certain levels, placing the corrsponding carriers in shallower levels or in other, as yet unexcited, deeper levels. These two trapping mechanisms were discussed earlier (section C.3). The effect of the prolonged irradiation on the photoconductive yield at the energy of irradiation may be

(a) A decrease due to a depletion in the number of centers which can be ionized by this quantum energy or

(b) An increase, in spite of (a), due to the fact 
that the density of trapping centers is decreased and hence the range increased.

The occurrence of (a) would required, of course, that the range be little affected by the filling of traps, i.e.. that the density of ionizable centers be much less than the density of trapping centers: Experimentally, however, (b) was observed for irradiation in either the 4.0 or $5.0 \mathrm{ev}$ bands. Thus it can be concluded that the depletion of the ionizable centers is more than offset by the increase in range afforded by the filling of the trapping centers. IfFrom this it follows that the density of trapping centers is less than the density of ionizable $(5 \mathrm{ev})$ centers and therefore that the latter cannot themselves provide the only trapping mechanism.

A similar conclusion follows from the effect of the prolonged irradiation on the photoconductive yield at quantum energies other than that of the irradiation. For the large relative increase that occurred at the lower energies (Figure 15) can be explained by the increased occupancy of levels which would be empty if the crystal were in thermal equilibrium.

The lack of obvious structure in the spectrum of Figure 15, curve $C$, may simply be due to presence of a number of overlapping bands. The experimental curves could probably be explained by the superposition of 4 or 5 bands with widths of 0.6 to $0.8 \mathrm{ev}$. The low energy section of curve $B$, however, does not appear to require the assumption of as many levels. This spectrum appears to show that no levels are filled which have optical excitation energies as low as some 
of those which can be filled by the $5.0 \mathrm{ev}$ irradiation. According to Figure 14, the efficiency of the activation increased abruptly when the energy of irradiation was changed from 3.4 to $3.9 \mathrm{ev}$. This, of course, is in agreement with the previously determined spectral dependence of photosensitivity (see Figure 6). The difference between curves 5 and 6 was not expected but in view of the fact that curves 5 and 6 were brought about by irradiations in the $5 \mathrm{ev}$ band, following previous irradiations in the $4 \mathrm{ev}$ band, we may expect the situation to be quite complicated. This will be especially true if excitation in the two bands does not lead to the same type of carrier. In fact, since the photoconductivity spectra induced by the two irradiations were not the same, it may be concluded that the two exeitations do not produce the same type of carrier. This conclusion is strengthened by the results of section $B .11$.

From the absorption change which occurred under $5.0 \mathrm{ev}$ irradiation (Figure 16) it is evident that such irradiation introduced the centers characteristic of excess oxygen ( $\mathrm{cf}$. Figure 4). Now according to the discussion to be given in section C.11, the 5.0 ev transition leads to free electrons, so that the only obvious transition which could lead to the formation of the excess oxygen centers is that indicated in Figure $31(\mathrm{e})$. This model associates the $5 \mathrm{ev}$ transition with a potential excess oxygen center. The excitation of this center by the absorption of a $5 \mathrm{ev}$ quantum and its subsequent 
thermal ionization would result in the level configuration of Figure $31(c)$ which we ascribed to the excess oxygen center.

It should be noted that the induced absorption shown in Figure 16 has a long tail extending well into the visible region. This part of the spectrum cannot be associated with the excess oxygen type centers. It is suggested that it can be ascribed to those centers which also provide the photoconductivity in this part of the spectrum (Figure 15, curve C). According to section C.II the irradiation at $4.0 \mathrm{ev}$ creates free holes. It is not possible to associate the origin of these holes with potential metal-excess centers, since the absorption spectrum of the latter is not very specific (Figure $9)$. Moreover, the absorption change on irradiation was sufficiently small that poor accuracy was obtained in these measurements (Figure 17). In any case, a comparison of Figures 9 and 17 shows that there is some difference between the irradiation induced absorption and that induced by heating in $\mathrm{Mg}$ vapor. All that can be said with certainty is that the holes produced by the $4 \mathrm{ev}$ radiation are not trapped to form oxygenexcess centers (Figure 17).

One other important feature of curve B, Figure 15, should be mentioned. Irradiation in the $4 \mathrm{ev}$ band reduced the photoconductive yield in the $5 \mathrm{ev}$ band. It seems reasonable to suppose that the holes, trapped in shallower levels after being excited by $4 \mathrm{ev}$ radiation, can then provide an additional trapping mechanism for electrons excited by 5 ev radiation. 
Thus the electron range would be reduced and the magnitude of the yield in the $5 \mathrm{ev}$ band would decrease as observed. To summarize, we suppose that irradiation in the $5 \mathrm{ev}$ band excites electrons to the conduction band and that at least some of these are subsequently trapped in a number of previously unoccupied levels (see Figure 3I(e). The electron range is thereby increased and in addition, since optical excitation of these shallow levels is now possible, photoconduction is observable at lower quantum energies. At the same time excitation to the states emptied by the irradiation becomes possible and an increase in optical absorption in the excess oxygen bands is observed (Figure 16).

On the other hand, irradiation in the $4 \mathrm{ev}$ band creates free holes which are then trapped by levels (Figure $31(f)$ previously occupied by electrons. The potential excess oxygen type centers do not, however, take part in the hole trapping process.

C.10 X-Ray Irradiation

The $X$-ray induced absorption spectrum of Figure 19, curve $A$, shows clearly the $5.7,4.3$ and $2.3 \mathrm{ev}$ bands. Weber ${ }^{10}$ has shown that the $4.8 \mathrm{ev}$ band is also present and this result has been confirmed by Sosheall. The latter author has also obtained evidence that there is more absorption to the high energy side of the $5.7 \mathrm{ev}$ peak than can be accounted for by a symmetrical band centered at $5.7 \mathrm{ev}$. It appears, therefore, that the $5.2 \mathrm{ev}$ band, found by Johnson 15 in excess oxygen crystals, may also be produced by X-irradiation. 
Thus the spectrum of Figure 19, curve A, contains all, or all but one, of the bands characteristic of excess oxygen plus some extra absorption lying in the visible region of the spectrum. The band at $2.3 \mathrm{ev}$ is easily defined but in the region between the latter and the $4.3 \mathrm{ev}$ band there is no obvious resolved structure. In fact, it has been shown, again by Sosheall, that after the thermal decay of the $2.3 \mathrm{ev}$ band there remains in the same spectral region some unresolved absorption of rather small magnitude. Consequently, it appears that the absorption, over and above that also found in excess oxygen crystals, consists of the $2.3 \mathrm{ev}$ band plus a small more or less continuous absorption extending from about $2 \mathrm{ev}$ to at least $3.6 \mathrm{ev}$. This may be due to the superposition of a number of relatively broad bands as suggested in the previous section inconnection with. UV excited specimens (cf. Figure 15).

As shown in Figure 20 most of the visible absorption decays rather rapidly at room temperature while the ultraviolet absorption decays rapidly at first then more and more slowly until the decay rate becomes immeasurably small. These bands were observed to the extent of 40 percent of their saturation intensity 4 months after their formation. Thus some of the centers appear to have higher thermal activation energies than others. This type of observation has been made previously in the case of X-rayed alkali halide crystals ${ }^{18}$. It has been variously assumed for the latter that (a) The thermal bleaching occurs by the thermal release 
of a trapped electron and its subsequent recombination with a trapped hole. Both the electrons and holes are believed to be connected with centers which evidence themselves in the absorption spectrum. Thus the decays of the absorption in different spectral regions must be correlated. An alternative decay mechanism is

(b) The recombination of trapped electrons and holes by the quantum mechanical tunneling process. The decay of the various absorption bands must again be correlated. With hypothesis (a) the large variation of decay rate with time has been taken to imply that the centers have different thermal ionization energies in spite of their common optical excitation energy. Seitz ${ }^{18}$, however, prefers hypothesis (b) and in this case explains the decay rate variation by a non-uniform distribution of trapped electrons and holes. Such a distribution of course, leads to a wide distribution in the distances between centers of opposite type and therefore also to large variations in the tunneling probability. Postulate (b), however, does not seem to explain in a simple fashion the commonly observed dependence of the thermal stability of the $\mathrm{X}$-ray induced centers on the temperature at which the irradiation is carried out. It is generally found, for example 19 , that samples irradiated at low temperature have a higher initial decay rate at room temperature than those irradiated at room temperature. A similar result was recently obtained by Sturtz ${ }^{20}$ for MgO crystals. The initial decay rate at room temperature was found to be much greater for crystals 
irradiated at that temperature than for those irradiated at higher temperatures. It is believed that these facts may be qualitatively explained by the model developed later in this section.

The effect of X-rays on the photoconductivity (Figure 21) was not correlated with the absorption change at high energies obtained by the same means (Figure 19). It seems reasonable to postulate that the photoconductivity is associated with the unresolved absorption discussed earlier in this section. Essshown in Figure 23, the photoconductivity spectrum induced by prolonged irradiation in the $5.0 \mathrm{ev}$ band is similar to that of an $\mathrm{X}$-rayed specimen which had undergone a partial thermal decay (by "similar" we imply that the spectra differ only by a factor which is independent of quantum energy). Since the former irradiation can only introduce one type of trapped carrier it is concluded that the partially decayed $\mathrm{X}$-rayed crystal also contains only one type of carrier capable of photoionization. The direct determination of carrier type (section C.11) shows that in both cases the spectra are due to trapped electrons. Thus the photoionizable trapped holes decay very readily at room temperature, at a rate which is comparable to that of the decay of the resolved absorption band at $2.3 \mathrm{ev}$ (Figure 22). It then seems reasonable to suppose that these trapped holes disappear by recombination with electrons from the $2.3 \mathrm{ev}$.centers. Since the optical activation energy of the latter is smaller than that for the trapped holes (Figure 15, curve B) it is also reasonable to 
suppose that the rate of decay of both types of centers is determined by the thermal ionization rate of the $2.3 \mathrm{ev}$ centers.

The disappearance of the trapped holes can, on the basis of previous considerations, be expected to have two other consequences. In section C.9 it was concluded that the production of trapped holes provided an additional trapping mechanism which appreciably reduced the electron range. Thus in the present case the decay of the trapped holes can be expected to increase the photoconductive yield in that region. of the spectrum where electronic excitation predominates, i.e. above 4.ev. Secondly, a decrease in yield can be expected at lower energies because the above discussed recombination of shallow trapped electrons with the trapped holes leaves fewer centers which can be ionized by quanta in this energy region. Both of the required features are shown by Figure 21 (compare curves $B$ and D).

After the relatively rapid processes discussed above have taken place the UV absorption bands can only decay by combination withithermally released electrons from the centers giving rise to the remaining photoconduction. Since the photoconductivity spectrum must be due to a number of different types of centers, the decay curves for the UV bands may be expected to be complicated. In fact since each type of trapped electron will be expected to decay monomolecularly with its own time constant, the decay curve should be resolvable into a number of simple exponential terms. An analysis of this type has been given for the decay of X-ray induced luminescence in $\mathrm{KBr}$. 
by Williams et al ${ }^{21}$, who apparently have located several levels in which carriers can be trapped. A similar analysis of the decay of optical absorption in $\mathrm{MgO}$ is shown in Figure 32 where 3 characteristic time constants are also given. In order to calculate thermal ionization energies from these, the value of $v_{0}$ in the expression 22

$$
\frac{1}{\tau}=v_{0} e^{-E / k^{T}}
$$

must be known. Here $r$ is the time constant to be associated with a thermal ionization energy $E$. Table IV shows the calculated values of $E$ for various assumed values of $v_{0}$.

\section{Table IV}

Calculated Thermal Ionization Energies of the Shallow Electron Traps

\begin{tabular}{rccc}
$\tau$ & $v_{0}=10^{9} \mathrm{sec}^{-1}$ & $V_{0}=10^{12} \mathrm{sec}^{-1}$ & $V_{0}=10^{14} \mathrm{sec}^{-1}$ \\
\hline $1230 \mathrm{hrs}$. & $.90 \mathrm{ev}$ & $1.07 \mathrm{ev}$ & $1.19 \mathrm{ev}$ \\
$44 \mathrm{hrs}$. & .82 & 1.00 & 1.10 \\
$5 \mathrm{hrs}$. & .76 & 0.93 & 1.05
\end{tabular}

Thus the traps which decay in reasonable times at room temperature have thermal activation energies in the vicinity of $1 \mathrm{ev}$. The optical ionization energies should be somewhat greater than these. We may estimate on the basis of various 
theoretical and experimental results ${ }^{23}$ that the optical energies should be grouped around $2 \mathrm{ev}$ for these, the shallowest traps, which can be filled by excitation at room temperature. Then the photoconductivity in an X-rayed (i.e., traps filled) specimen should begin to drop sharply in this energy region. Figure 22 shows that this is approximately so.

Those traps which have thermal ionization energies appreciably greater than 1 ev (optical energies greater than about $2 \mathrm{ev}$ ) do not decay to a measurable extent at room temperature. Consequently, neither the magnitude nor the spectral dependence of the photoconductivity in the high energy region would be expected to change with time, once the trapped holes and the shallow trapped electrons have essentially disappeared.

To summarize, the proposed model attributes the large increase IIn photoconductive yield induced by $\mathrm{X}$-irradiation to

(a). A large increase in $x$ (see section B.2. for the meaning of the symbols) because many of the electron and hole traps are filled and

(b) An increase in the $\dot{p}_{i} K_{i}$ because excitation from the newly filled levels becomes possible. The change in spectral dependence (curves B, C, D, Figure 2I) with thermal decay is attributed to the liberation of trapped holes which also annihilate the electrons which gave the $2.3 \mathrm{ev}$ band absorption (Figure 22). The fact that the yield at high energies actually increases during the thermal decay (curve D, Figure 21) may be evidence that the trapped holes act 
as electron traps as previously suggested. An increase in $x$ for electrons would then occur as the holes decayed.

The experiment illustrated by Figure 23 is in accord with the above model in that it shows that a specimen excited in the $5.0 \mathrm{ev}$ band (curve $A$ ) can be returned to the same state $C$ (except for an increase in $x$ after a subsequent $\mathrm{X}$-irradiation, by simply allowing a few hours of thermal decay to take place.

The fact that iradiation at 2.3 ev reduced the photoconductive yield by approximately the same fractional amount at all energies (Figure 26) implies that the reduction is due to a decrease in $x$. This decrease presumably occurs because the 2.3 ev quanta ionize some of the shallow filled levels thus providing more levels for trapping. The ellectrons from the se levels can recombine with the trapped holes in the excess oxygen type levels (Figure $31(\mathrm{c})$, thus reducing the optical absorption in the 5.7, 4.8 and $4.3 \mathrm{ev}$ bands (Figure 24). Since the photoconductive yield at $2.3 \mathrm{ev}$ was not preferentially bleached, it must be concluded that only a small fraction of the filled levels of this energy were emptied in the course of this experiment. The few extra empty levels must, of course, increase the trapping probability sufficiently to decrease the range by 60 percent (Table III).

Although Figure 25 shows a rather large fractional decrease in absorption at $2.3 \mathrm{ev}$, it does not contradict the statements made above in connection with Figure 26. The crystal of Figure 25 was freshly $\mathrm{X}$-rayed and therefore contained a prominent 
absorption band at $2.3 \mathrm{ev}$ ( $\mathrm{cf}$. Figure 29, curve A). It is believed that the absorption decrease shown in Figure 25 is due principally to the disappearance of this band. In the case of the partially decayed crystal, however, this band has already decayed thermally to a very low level. The bleaching of the photoconductivity by quanta of this energy is then attributed to the excitation of electrons from levels which are evidenced by the previously discussed (Section C.9) unresolved absorption superimposed on the $2.3 \mathrm{ev}$ absorption band (Figure 16).

\section{C.11 Sign of the Charge Carriers}

In order to provide an interpretation of the results presented in section B.II, some discussion of the space charge field distribution will be given for a very idealized case.

Consider the experimental situation as illustrated by Figure $3(\mathrm{c})$ wherein the ZY planes constitute the electrodes of a crystal with the light beam incident on the XZ plane and illuminating a fraction of the volume, $\underline{b}$ wide as shown in the figure. Assuming that the distance moved by each excited carrier is small compared to $a_{1}, a_{2}$ and $b$, the result of an irradiation, during which the electric field is applied, is to give the field distribution shown by the solid line in Figure 33(a). The dotted line shows the field before irradiation, i.e., that which would be calculated from the applied voltage and the electrode separation. The second step in this idealized experiment is to 
remove the applied voltage and bring the electrodes to the same potential. The field distribution is then as shown in Figure $33(\mathrm{~b})$ which is simply a vertical displacement of the previous diagram.

For definitness it is assumed at this point that the excited carriers are electrons. The discussion can be easily altered to fit the case of hole excitation. In this case the field distributions of Figures $33(a)$ and $(b)$ are the result of the space charges indicated on the diagrams. The important fact here is that the electron excess lies outside the illuminated region while the electron deficiency lies within this region.

The third step of the experiment consists in the excitation of more carriers (within the same volume as previously irradiated) and their motion under the influence of the space charge field alone. Under these conditions the motion of the excited electrons is to the right and the tendency is for the former electron deficiency to be neutralized and a new deficiency to form at the left hand side of the irradiated region. (Notice that the electron excess is not disturbed because it lies outside the irradiated region.) If this process could be carried to completion the space charge distribution would be as shown in Figure 33(c) with the corresponding electric field having an appreciable value only at the left hand edge of the illuminated region. If the photocurrent were carried by holes rather than by electrons the "dipole" layer would exist at the opposite edge of the 
illuminated region (i.e., the edge nearest the electrode which was held negative during the first step of the experiment). According to the foregoing discussion the sign of the charge carriers can be determined if the dipole layer can be located. This can be done in practice by moving a narrow beam of light across the crystal and measuring the photocurrent as a function of the beam position. If the electrodes are at the same potential during this "scanning" the photocurrent observed will be due to the motion of carriers in the previously developed space charge field and hence will be a measure of this field. The measured dependence of photocurrent on beam position will, of course, only be a true representation of the electric field distribution if the sensitivity of the crystal is uniform; that is if the UV irradiation has not appreciably activated the crystal. This condition very likely cannot be met in practice but nevertheless this effect does not interfere with the location of the dipole layer.

In an actual experiment the results may be expected to deviate from those shown schematically in Figures $33(a)$ to (c). There are several obvious reasons for this;

(a) The carrier range may not be small compared to the width of the irradiated region.

(b) The carrier range may vary during the course of the experiment by means of the activation effect discussed above and also because the electric field is not constant during the experiment.

(c) The term, "range", as used above should actually read "mean range" since the displacement of a given electron 
can vary between zero and a distance comparable to the distance between the electrodes,

(d) The width of the scanning beam is not negligible compared to the width of the irradiated region,

(e) It is difficult to estimate the optimum duration of the second irradiation (no applied field). If insufficient time is employed the field due to the dipole layer may be masked by that due to the unneutralized charges at either edge of the illuminated region. If the second irradiation is prolonged to avoid the former effect, the dipole itself may be destroyed, by the continuous dark current, for example.

In spite of the above listed difficulties, it is believed that the results of section $B .11$ may be used to determine the sign of the charge carriers excited under various conditions. For, even if a clearly defined field minimum, corresponding to the "dipole" of Figure $33(\mathrm{c})$, cannot be discerned, the location of the "dipole" can be deduced from the asymmetry of the field distribution. Thus in Figure 27, curve $B$, the field minimum was shifted towards the negative electrode by the formation of the dipole layer. It is therefore concluded that excitation in the 4.0 ev band creates free holes.

In Figure 28, curve $B$, there is no obvious minima corresponding to that of curve A. However, since further irradiation (curve C) brings out the left hand minimum preferentially, we ascribe the latter to the dipole layer. Thus the dipole was formed nearest to the positive electrode and it is concluded that excitation in the 5.0 ev band creates free electrons. 
The interpretation of Figure 29 is more obvious since the right hand minimum of curve $B$ is readily associated with that of curve $A$. Thus, the left hand minimum of curve $B$ is ascribed to the dipole and it is concluded that 4.4 ev quanta create free electrons in a partially decayed X-rayed crystal. 


\section{CONCLUSIONS}

Much of the material presented here consists of preliminary investigations of the optical absorption and photoconductivity in MgO crystals of inferior purity. The interpretation of the results required the introduction of a large number (or even continua) of energy levels in the normally forbidden gap. It is recognized that interpretations made on such a basis are not very satisfying. However, it should be pointed out that even in some other, much more thoroughly explored areas (e.g. the properties of CdS and similar photoconductors ${ }^{24}$ ), similar postulates are found to be necessary. Furthermore, the observed properties were found to be reasonably reproducible among the several lots of MgO available. Thus it is believed that these properties, although they are probably determined to a large extent by the large impurity content, should also apply to many of the specimens for which data appear in the literature.

The following are the more important tentative conclusions resulting from this work.

(1) The most prominent UV absorption bands -- those characteristic of excess oxygen and occurring at $4.3,4.8$, and $5.7 \mathrm{ev}-$ have no photoconductivity associated with them (section 6). (2) The photoconductivity in stoichiometric or excess oxygen crystals lies principally in two Gaussian shaped peaks centered at $4.05 \mathrm{ev}$ and $5.05 \mathrm{ev}$. Excitation in these peaks is found to result in free holes and free electrons respectively. (Sections 
6 and 11).

(3) Addition of an excess of Mg to stoichiometric crystals reduces or eliminates the optical absorption characteristic of the stoichiometric state: (Section 8) For this reason it is tentatively concluded that the absorption spectra of stoichiometric crystals and of excess Mg crystals are associated with the same centers (Figure 31(d). With this model the background absorption is interpreted as being due to the transitions show in the figure. The addition of excess $\mathrm{Mg}$ is then considered to provide electrons which fill the upper states, thus eliminating the possibility of the transitions shown and therefore reducing the background absorption. At the same time transitions from the upper states to the conduction band are made possible, thus providing the extra absorption and photodonductivity observed in excess Mg crystals. It seems necessary to postulate a near continuum of levels to explain the absorption in either stoichiometric or excess Mg crystals and also to explain the photoconductivity of the latter.

(4) Excitation of free electrons results in the filling of a number of shallow levels (Figure $31(e)$ some of which seem to contribute strongly (when empty) to the electron trapping process (section 9). Those of high energy (with excitation energies greater than say $3 \mathrm{ev}$ ) may be those which are also filled by the addition of excess Mg. The lower energy ones are believed to be responsible for the absorption in the visible regions of Figure 16 (irradiated in $5 \mathrm{ev}$ band) and for part of that in the visible region of Figure 19, curve $A$ $(X$-rayed). 
(5) Irradiation in the $5 \mathrm{ev}$ band introduces the absorption bands characteristic of excess oxygen. The 5 ev transition is believed to be associated with a potential center of the latter type. (Figure $3 I(e)$.

(6) The excitation of free holes (e.g. by $4 \mathrm{ev}$ band irradiation) causes a small increase in UV absorption. No structure is obvious in this spectrum. Some levels lying nearer to the valence band than the $4 \mathrm{ev}$ level trap these holes, making possible photoconduction at somewhat lower energies. (Section 9). These trapped holes can act as electron traps so that as they are formed, the efficiency of electronic photoconduction decreases (Figure 15) and as they are thermally ionized the electronic yield increases (Figure 21, curve D). (7) X-irradiation excites both electrons and holes and provides the following changes in level occupancy.

(a) The upper filled levels of Figure $31(e)$ are emptied thus providing the excess oxygen absorption bands at '5.7. 4.8 and $4.3 \mathrm{ev}$ and at the same time decreasing the number of 5.0 ev excitations (Figure 21).

(b) The series of shallow levels of Figure $31(e)$ is filled with electrons thus increasing the range of free electrons and providing optical absorption in the visible region of the spectrum. Part of this absorption lies in a well defined band (Figure 19) while the remainder shows no obvious structure and corresponds to the photoconductivity of the crystal after a partial thermal decay (Figure $21(d)$. (c) The series of low lying levels of Figure $31(f)$ are 
emptied thus providing photoconductivity at lower energies (Figure 21 , curve B) and at the same time providing additional electron traps.

(8) The thermal decay proceeds by:

(a) The thermal release of electrons from the centers which give rise to the 2.3 ev absorption band. These electrons then combine with the trapped holes in the levels of Figure $31(f)$. By this means the hole photoconductivity is reduced and the electron range increased (compare curves $B$ and $D$, Figure 21), while the visible absorption decreases.

(b) The UV absorption centers (Figure 31 (c) decay by combination with electrons thermally released from the series of levels of Figure $31(e)$. Since a number of thermal activation energies are involved in this release, the dependence of UV absorption on time can be decomposed in a number of simple exponential terms (Figure 32 ).

(9) Bleaching of the UV absorption may be accomplished by using any wave length which can excite electronic photoconduction, i.e., any energy from about $2 \mathrm{ev}$ to about $6 \mathrm{ev}$ or higher. The higher energies may however, produce complicating side effects. When the bleaching radiation lies in the $2.3 \mathrm{ev}$ absorption band electrons may be excited either from the levels responsible for this band or from those which also lie at this energy but are part of the quasi-continuous distribution shown in Figure $31(e)$. Thus, in the bleaching experiment described in section $\mathrm{B} .10$, no simple relationship between the absorption changes in the $2.3 \mathrm{ev}$ band and in the UV absorption bands may 
be expected.

(10) Bleaching of the $X$-ray induced photoconductivity can be achieved by irradiation with $2.3 \mathrm{ev}$ quanta. The effect is due to the emptying of electron traps by optical ionization as discussed above and the consequent reduction in electron range. 


\section{E. COMPARISON WITH PREVIOUS WORK}

The only previously reported measurements of photoconductivity in MgO crystals were made by Day ${ }^{5}$. Since the two sets of results are in conflict on a number of points Day's results will be discussed in some detail. The most important differences are:

1. The peaks at 3.6 and $4.8 \mathrm{ev}$ obtained by Day in neutron irradiated crystals were not found in $X$-rayed crystals in the present work. This fact alone is not cause for alarm, but it does seem reasonable to expect that any means of excitation which is capable of exciting both electrons and holes will result in the formation of similar absorption and photoconductivity bands. The apparent discrepancy can, however, be resolved by a consideration of the geometry used by Day and the fact that the correction factor $\mathrm{K} / \mathrm{a}$ (equation (1) was omitted in his calculations. The dimension of his crystal in the direction parallel to the light beam was about $1 \mathrm{~cm}$. Thus the quantity $\underline{\text { a }}$ (equation (2) achieved a constant value at relatively low quantum energy (say about $3.6 \mathrm{ev}$ ) compared to the case of a thinner specimen where the constancy of a would not be achieved until the quantum energy was well into the $4.3 \mathrm{ev}$ optical absorption band. The spectrum (Day, ref. 5, Figure 1) should therefore be multiplied by a factor proportional to $K$ for quantum energies greater than $3.6 \mathrm{ev}$. Since $\mathrm{K}$ is expected to peak at $4.3 \mathrm{ev}$ (Figure 22) the "valley" at $4.3 \mathrm{ev}$ in Day's spectrum would tend to be 
eliminated and the 3.6 and $4.8 \mathrm{ev}$ peaks thus disappear. The spectral dependence of photoconductivity would then essentially agree with the results of the present measurements. 2. The failure to recognize in the earlier work ${ }^{5}$ that the photoconductivity in stoichiometric crystals lies in two bands. The UV activation experiments were performed with a quantum energy of $4.0 \mathrm{ev}$. According to Figure 6 the activation obtained by this UV irradiation should have been due in part to absorption in the $4 \mathrm{ev}$ band and in part to $5 \mathrm{ev}$ band absorption. The spectral dependence of the photoconductivity in the activated crystal (ref, 5, Figure 2) shows that the activation obtained was more characteristic of 5 ev band irradiation.

3. The sign of the charge carriers released by 4 ev irradiation. Day attempted to determine the sign of the charge released by $3.7 \mathrm{ev}$ radiation by detecting the displacement by the electric field of a narrow UV activated region of the crystal. The location of the activated region was determined by scanning the activated crystal with a narrow light beam. The procedure involved the implicit assumption that a plot of photocurrent versus distance across the crystal (in a direction parallel to the field) comprised a determination of the profile of the photosensitivity. However, since the irradiation was of necessity performed with the electric field applied to the crystal, space charge fields were presumably developed as discussed in section 5. Therefore, the variation of photocurrent with distance is a measure of the profile of the product 
of electric field and photosensitivity. Thus the interpretation of this type of experiment is confused by the formation of space charge fields.

In spite of the above objection Day's conclusion that the carriers produced by 3.7 ev irradiation are holes is in agreement with the result of section B.ll. However, his conclusion that the carriers excited during his activation experiment were therefore also holes is not warranted (activating irradiation was at $4.0 \mathrm{ev}, i . e$. in the tail of the $5 \mathrm{ev}$ band) and in fact, according to the present work, incorrect.

The optical absorption spectrum of the excess Mg crystal shown by Figure 9 is similar to that obtained by Weber ${ }^{10}$. The latter author, however, believed that this spectrum could be decomposed into 3 bands centered at $4.8,3.6$ and $2.3 \mathrm{ev}$. The structure is, however, not obvious in Weber's data and it is believed that he was misled on two counts.

(a) The decreasing background absorption discussed in section C. 8 makes the calculated induced absorption fall off in the range of quantum energies. where the background absorption has, before treatment, an appreciable magnitude (see Figure 12, curve $A$ ). If the induced absorption is nevertheless calculated in this way, it is possible to postulate a $4.8 \mathrm{ev}$ absorption band, whereas the true induced absorption would show no such falling off at high energies and would therefore not indicate the presence of such a band.

(b) Weber was also convinced that the absorption spectrum 
of an excess Mg crystal should consist of those bands which are found in $X$-rayed crystals but not in excess oxygen crystals. Since he was not aware that the $4.8 \mathrm{ev}$ band was present in excess oxygen crystals he believed that it should be present in excess Mg crystals since it was quite obvious in $X$-rayed crystals.

Having, attributed the absorption at high quantum energies in excess Mg crystals to the $4.8 \mathrm{ev}$ band, Weber could then decompose the remainder of the spectrum into 3.6 and $2.3 \mathrm{ev}$ bands, both of which he believed were present in the X-ray induced spectra. There seems to be no doubt about the existence of an absorption peak at $2.3 \mathrm{ev}$ in $\mathrm{X}$-rayed crystals. However, there seems to be no good evidence for its existence in excess Mg crystals nor for the existence of a 3.6 ev band in crystals treated in any fashion. It is believed that only the erroneous assumption of a $4.8 \mathrm{ev}$ band in the excess $\mathrm{Mg}$ crystals would lead one to postulate the existence of the 3.6 ev band.

Hibben ${ }^{25}$ found that MgO crystals were visibly colored by exposure to $4.9 \mathrm{ev}$ radiation. This is presumably the same effect as illustrated by Figure 16.

Eisenstein ${ }^{26}$ studied the $\mathrm{X}$-ray induced phosphorescence of MgO crystals. An emission band at $3.6 \mathrm{ev}$ was found to have a very long decay time. This band may be connected with the decay of the excess oxygen type absorption centers. The long decaytime:isoconisistent with the long term absorption decay shown by Figure 20. 
Yamaka and Sawamoto ${ }^{27}$ have determined the sign of excited carriers by measuring the Hall effect. It does not seem profitable to discuss their work in terms of the present results since no attempt was made to determine which photoconductivity bands were present in their samples. Radiation of a particular wavelength (provided by filters) was assumed to provide excitation in a "band" centered at the same energy. In this manner they determined that excitation in the 4.8, 3.6 and $2.3 \mathrm{ev}$ "bands" provided hole conductivity in excess Mg crystals. Although this conclusion is in doubt in view of the doubt concerning the presence of these bands in such crystals, the fact that hole conduction was detected is in contradiction to the discussion of Section C.II.

Yamaka $^{28}$ detected, by measurements of thermoluminescence, the presence of a number of traps whose thermal activation energies he estimated to fall in the region .56 to $1.58 \mathrm{ev}$. Since the same results were obtained both by X-ray and $4.9 \mathrm{ev}$ optical irradiation the traps are according to the present work electron traps. These are presumably the traps which are responsible for the photoconductivity in $\mathrm{X}$-rayed and partially decayed crystals (Figure 22, curve B, and Table IV). 


\section{BIBLIOGRAPHY}

1. Lander, Rev. Sci. Inst., 24, 331 (1953).

2. Strong and Brice, J. Opt. Soc. Am., 25, 207 (1935).

3. Jenkins and White, Fundamentals of Physical Optics, (McGraw-Hill, 1937) p. 294.

4. Seitz, Rev. Mod. Phys., 26, 44 (1954).

5. Day, Phys. Rev., 91, 822 (1953).

6. Geller, Phys. Rev., 101, 1685 (1956).

7. Hall, Phys. Rev., 87, 387 (1952).

8. Rose, Proc. IRE, $\underline{43}, 1850$ (1955).

9. Bube, Proc. IRE, 43, 1846 (1955).

10. Weber, Zeits f. Physik, 130, 392 (1951).

11. Soshea, M. S. Thesis, Electrical Engineering Department, University of Minnesota, (1956).

12. Molnar and Hartman, Phys. Rev. 91, 1015 (1950).

13. Lye, Scientific Report No. 1, U. S. Air Force Contract No. 33(616)-3325 (1956).

14. Blakney and Dexter, Defects in Crystalline Solids (The Physical Society, London, 1955), p. 108; Phys. Rev. 96. 227 (1954).

15. Johnson, Phys. Rev. 94, 845 (1954).

16. Krumhansl, Photoconductivity Conference (Wiley, 1956) p. 455 .

17. Seitz, Rev. Mod. Phys. 26, 53 (1954).

18. Ibid, p. 68

19. Ibid, p. 68, Fig. 34.

20. Sturtz, Electrical Engineering Department, University of Minnesota; private communication.

21. Williams, Usiskin and Dekker, Phys. Rev. 92, 1398 (1953).

22. Mott and Gurney, Electronic Processes in Ionic Crystals, Oxford, 1948), p. 130.

23. Lehovec, Phys. Rev. $\underline{92}, 253$ (1953). 
Vașileff, Phys. Rev. 96, 603 (1954).

24. Rose, Proc. IRE 43, 1857 (1955).

25. Hibben, Phys. Rev. 51, 530 (1937).

26. Eisenstein, Phys. Rev. 94, 776 (1954).

27. Yamaka and Sawamoto, Phys. Rev. 101, 565 (1956).

28. Yamaka, Phys. Rev. 96, 293 (1954).

29. Mott and Gurney, Electronic Processes in Ionic Crystals, (Oxford, 1948) p. 82 .

30. Seitz, ref. 4.

Burstein et al., ref. 16, p. 384.

31. Lax, ref. 16, p. 111.

Pekar, Z. expt1. teor. phys., 20, 267 (1950).

32. Burstein et al., Photoconductivity Conference (Wiley, 1956) pp. 379-382.

33. Bernard V. Haxby, Electrical Engineering Department, University of Minnesota, private communication.

34. Lipson et al., Phys. Rev. 99, 444 (1955). 
MOUNTING OF THE CRYSTAL FOR PHOTOCONDUCTIVITY MEASUREMENTS
SCHEMATIC ARRANGEMENT OF THE PHOTOCONDUCTIVITY APPARATUS

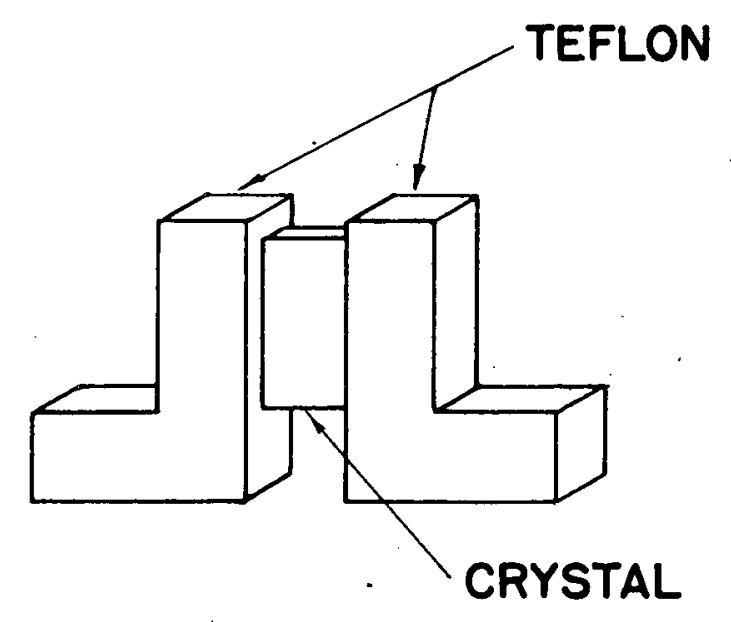

FIG. 2

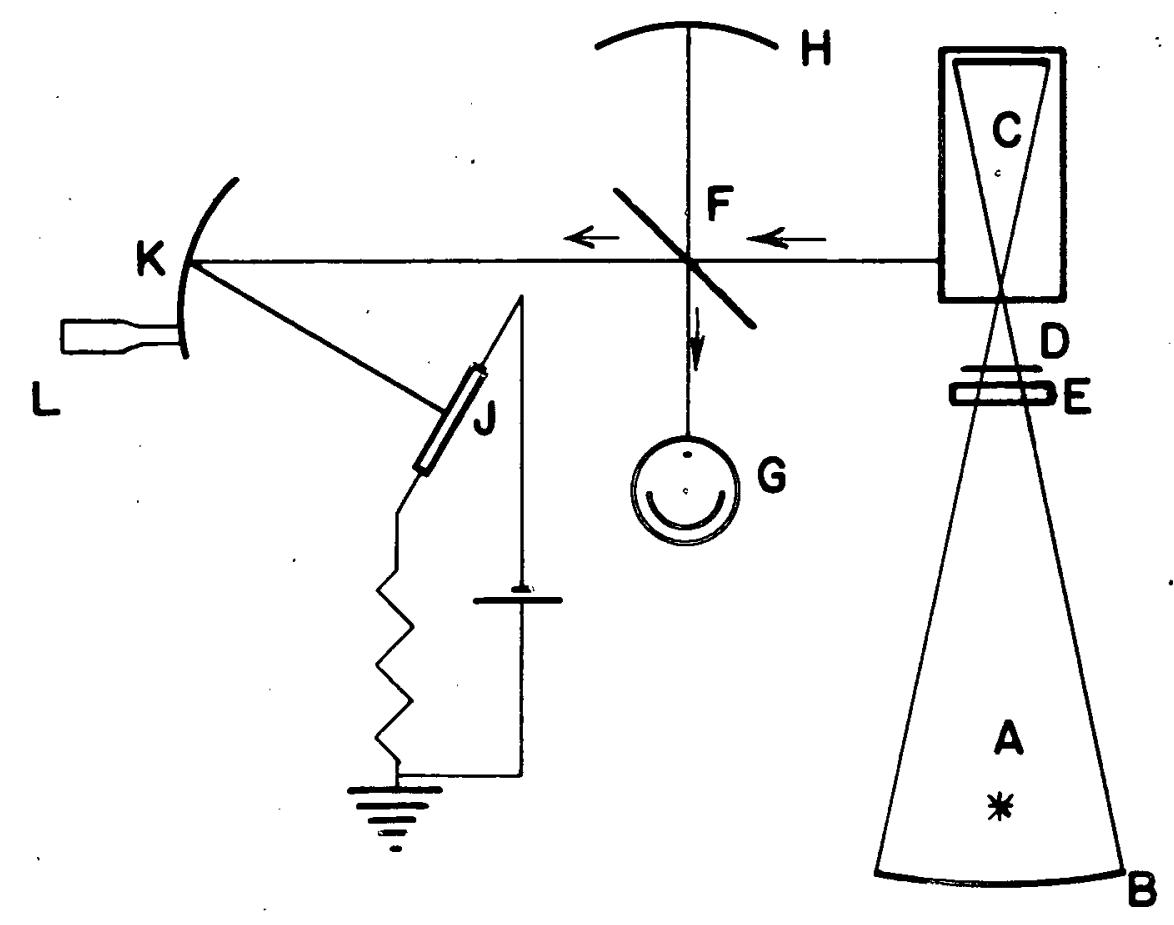

FIG. I 

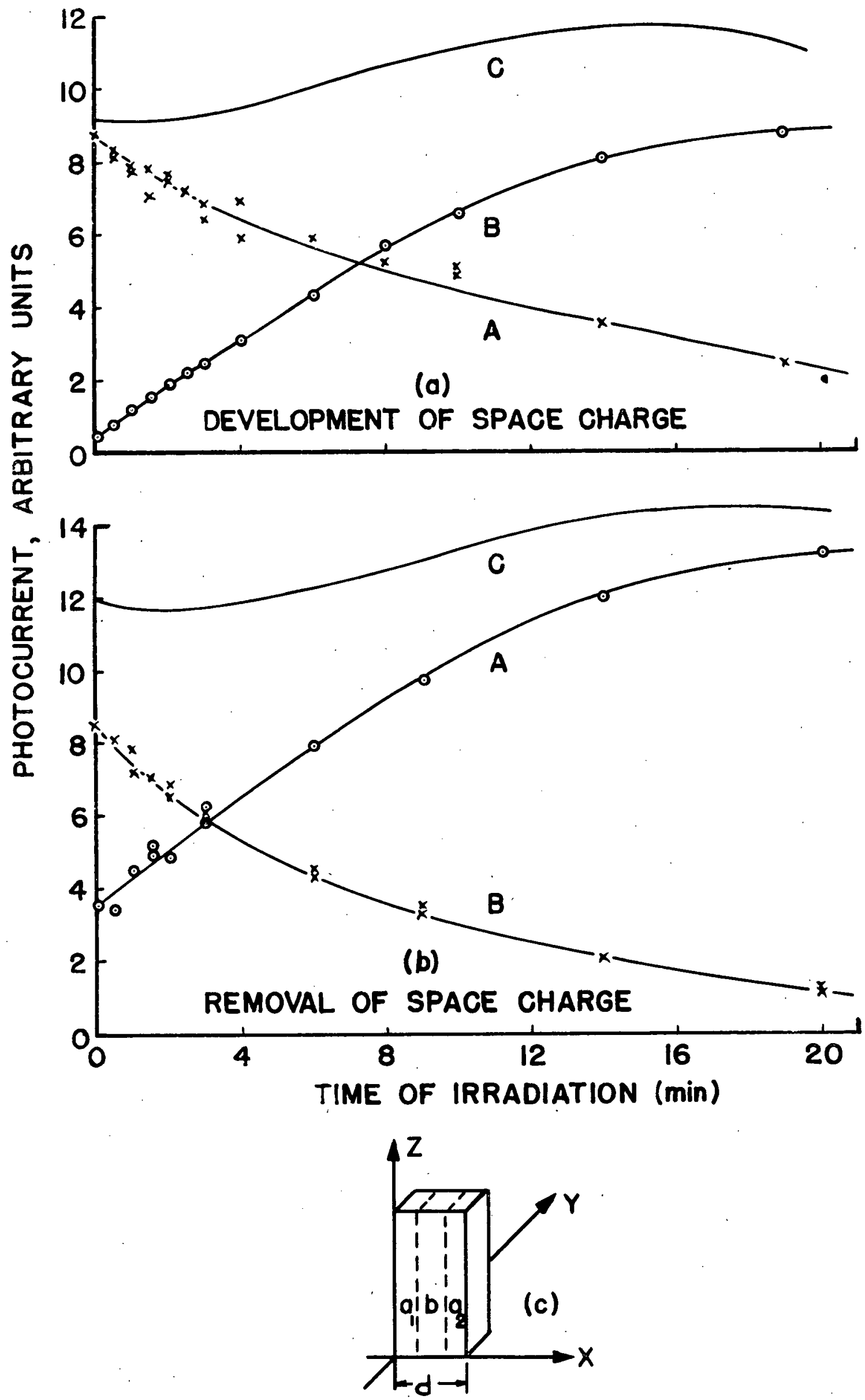

FIG. 3 


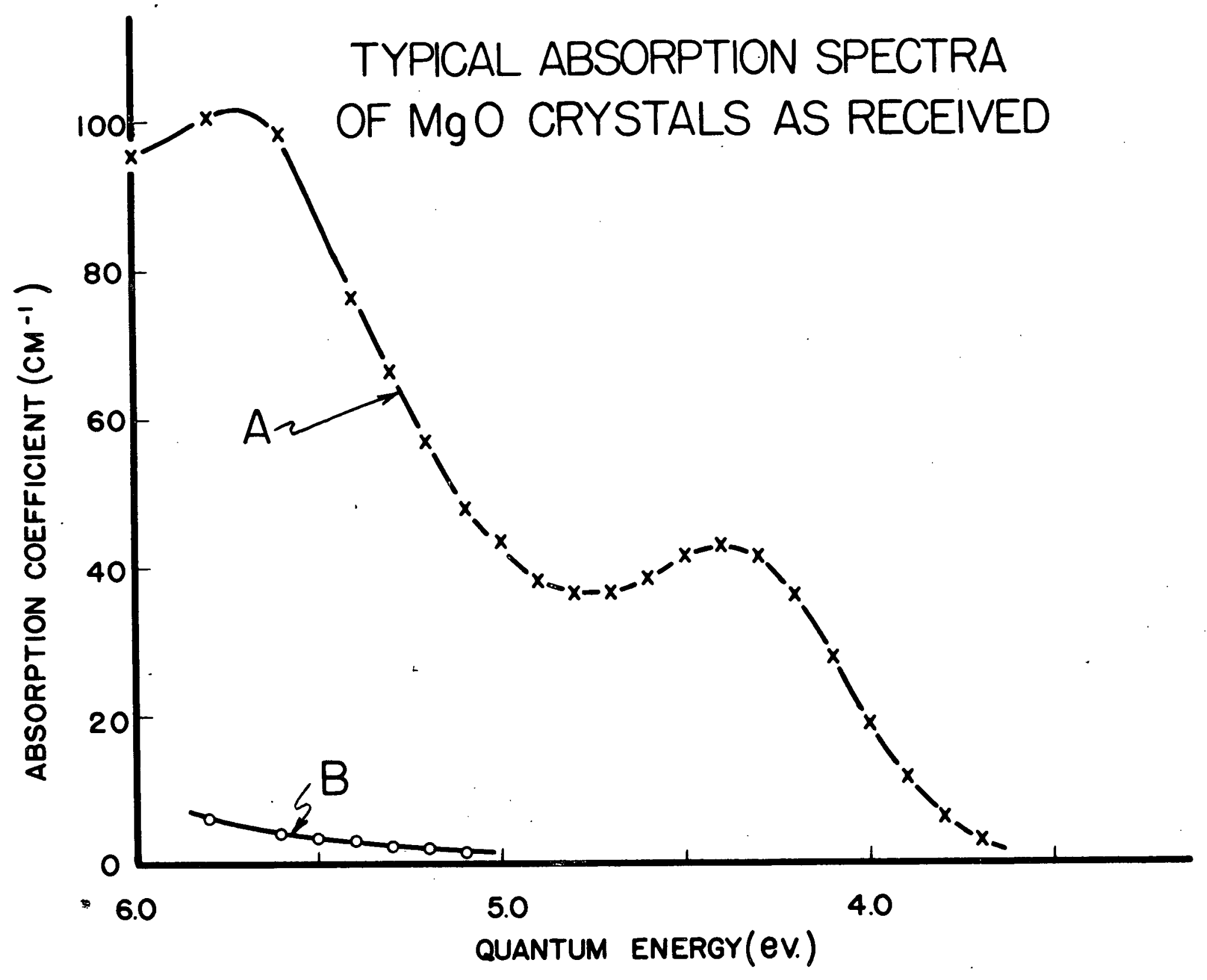

FIG. 4 


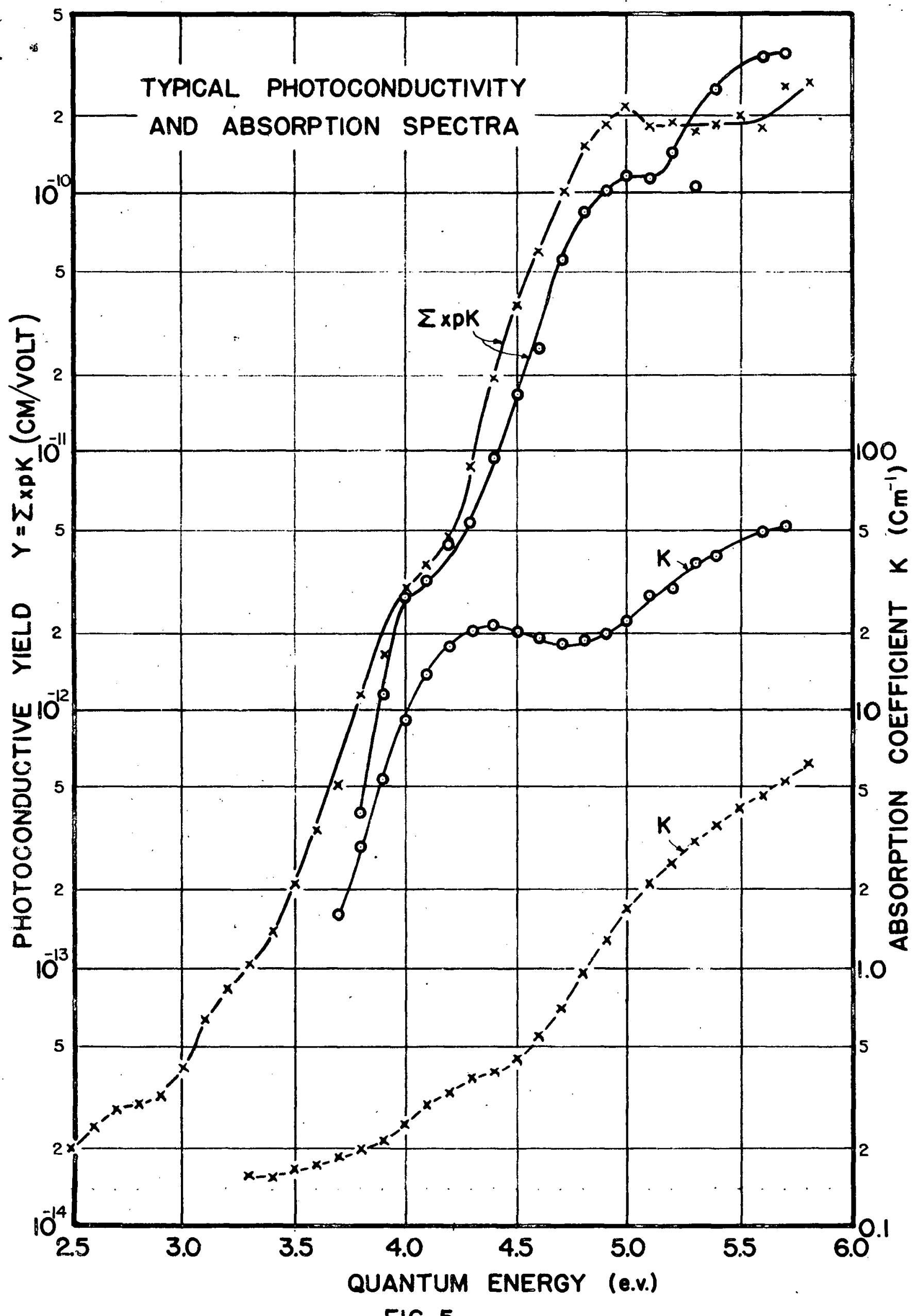

FIG. 5 


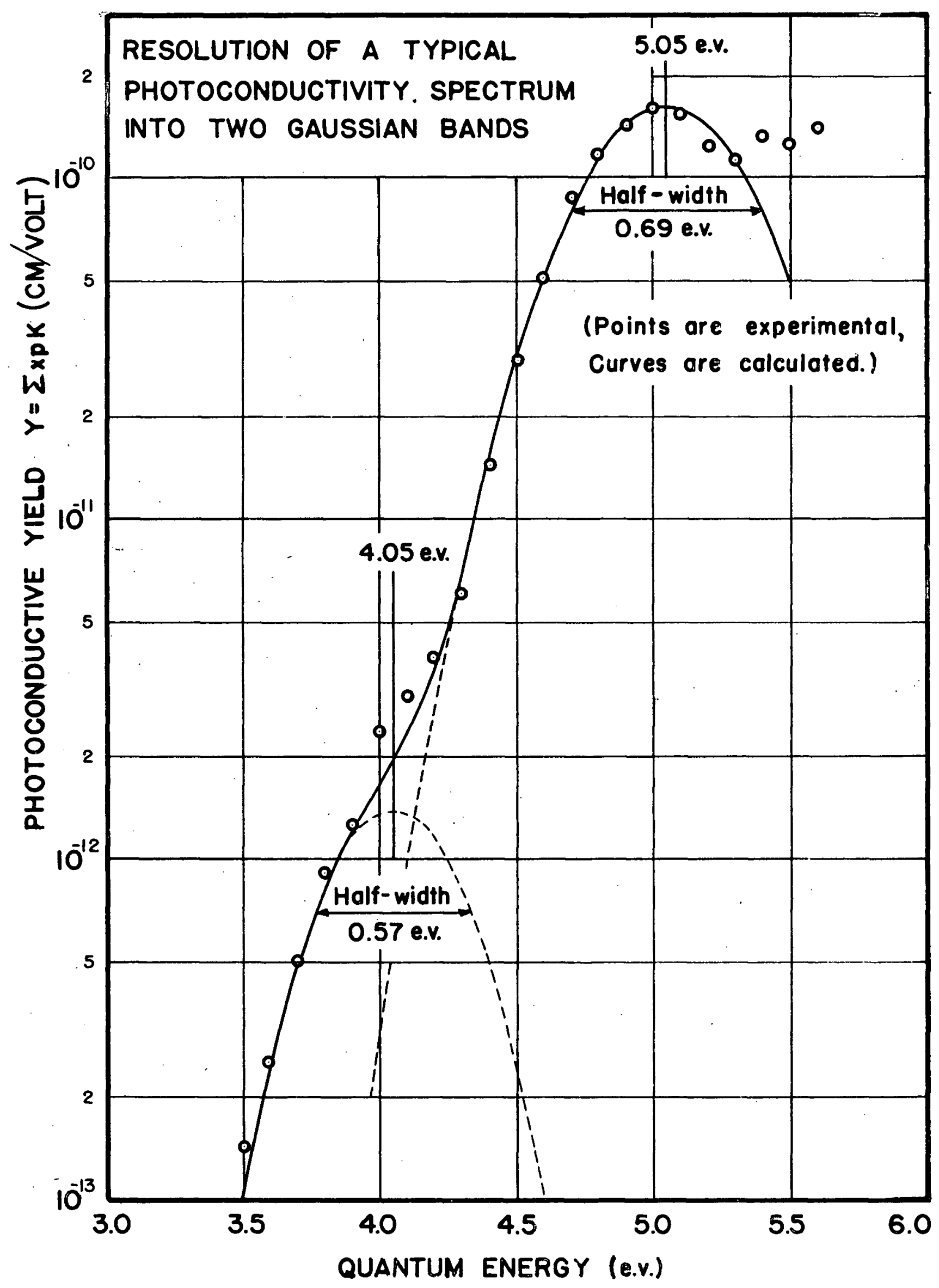

FIG. 6 
EFFECT OF HEAT TREATMENT ON THE BACKGROUND ABSORPTION

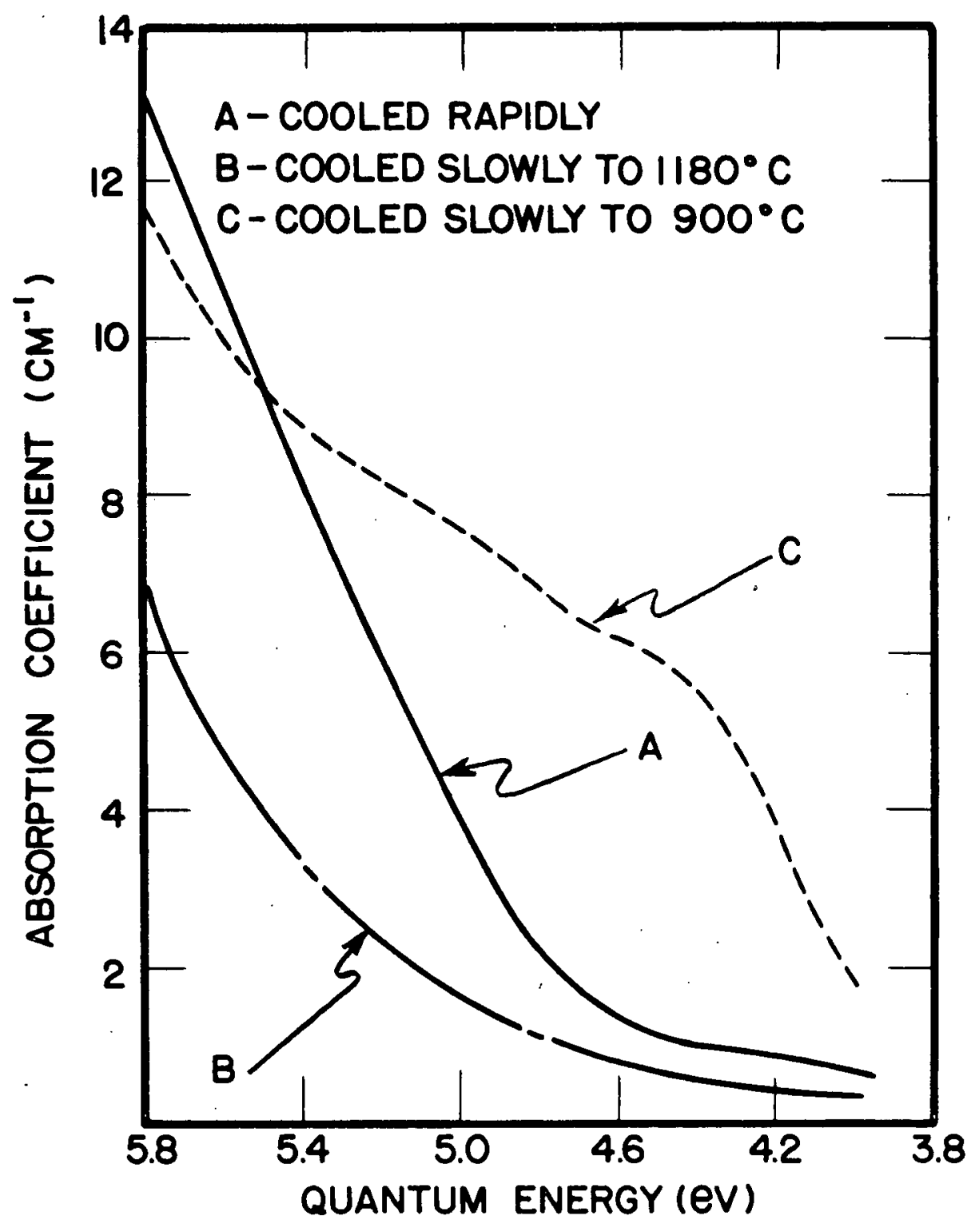

FIG. 7 


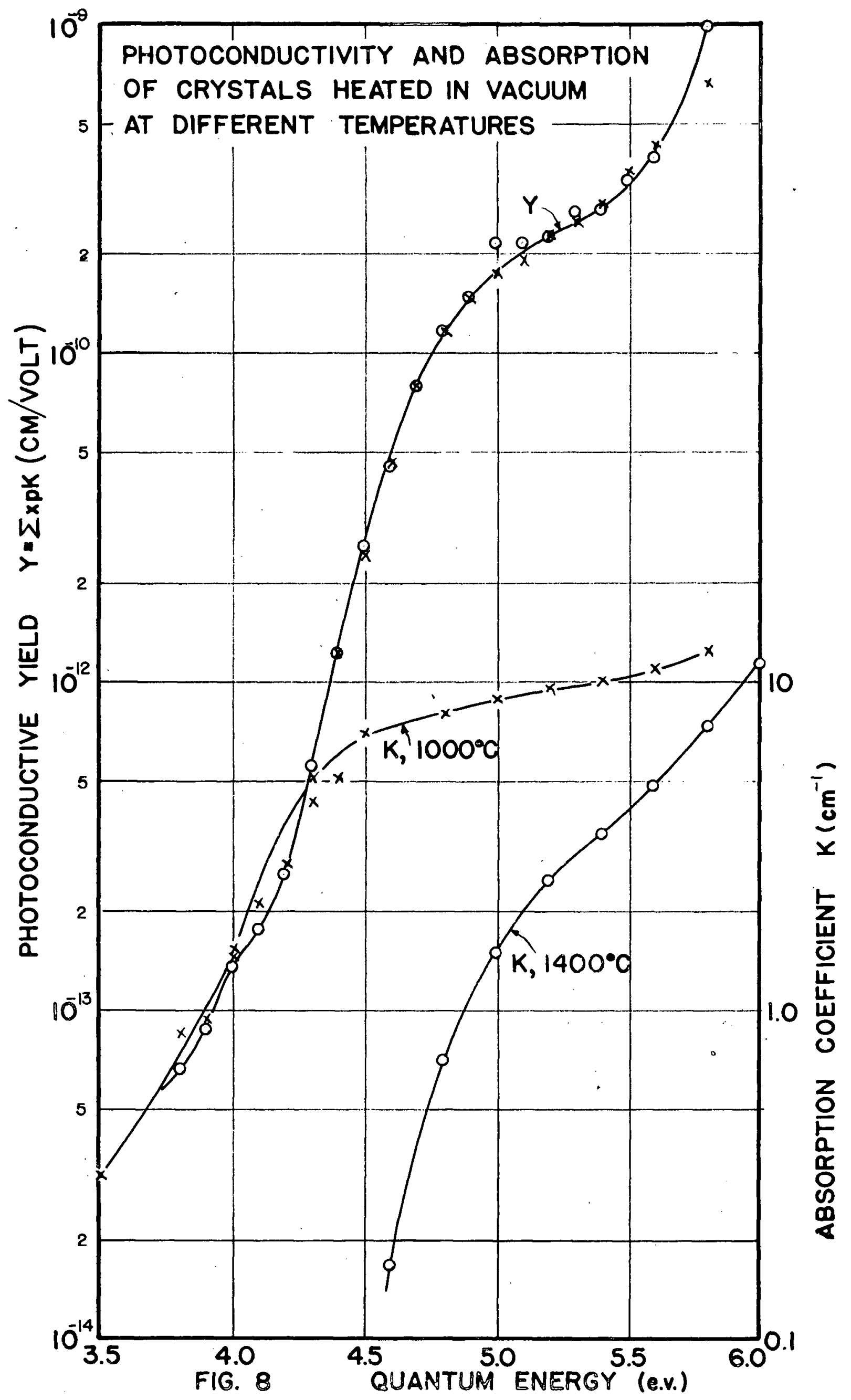




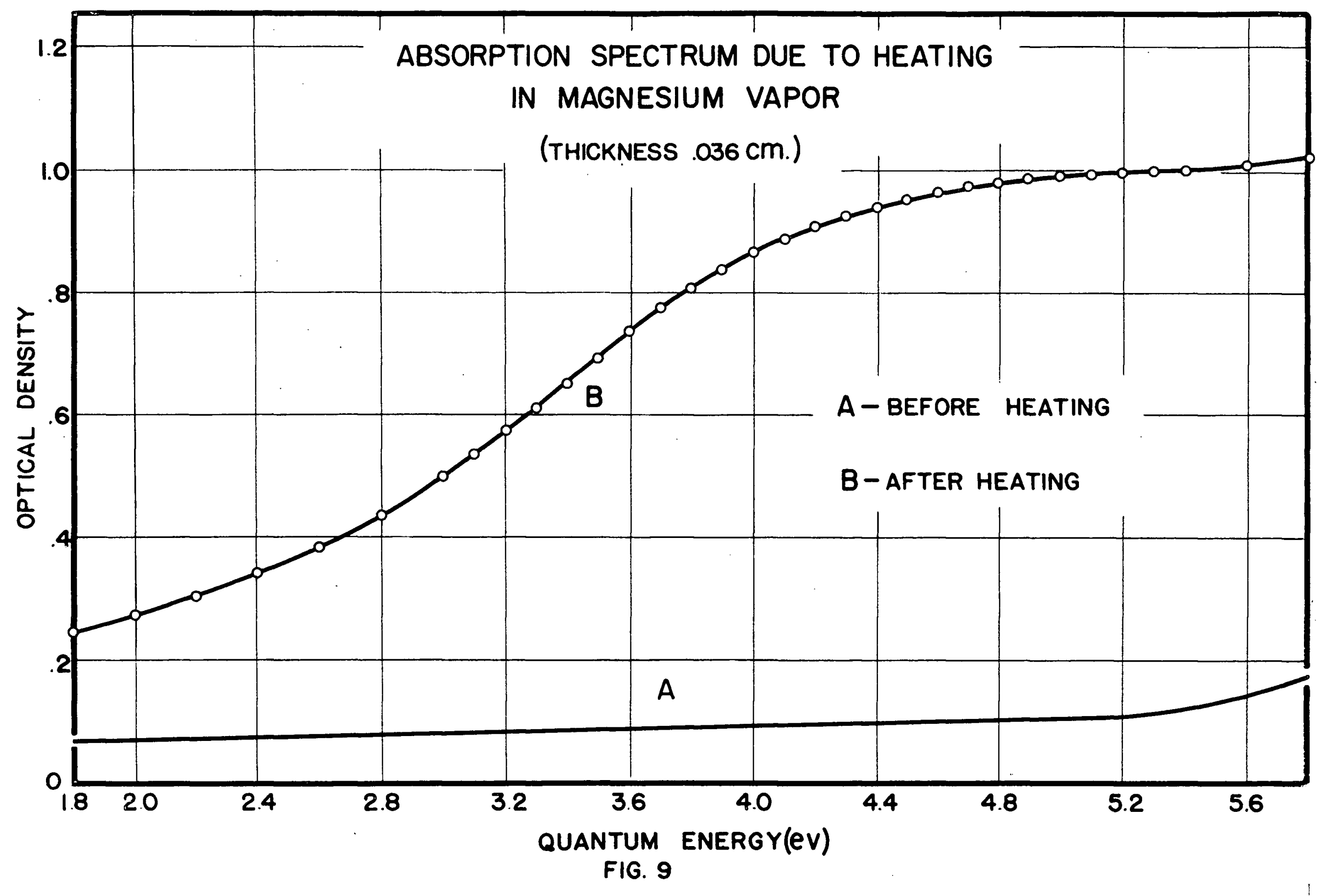


THE EFFECT OF EXCESS Mg ON THE BACKGROUND ABSORPTION OF MgO

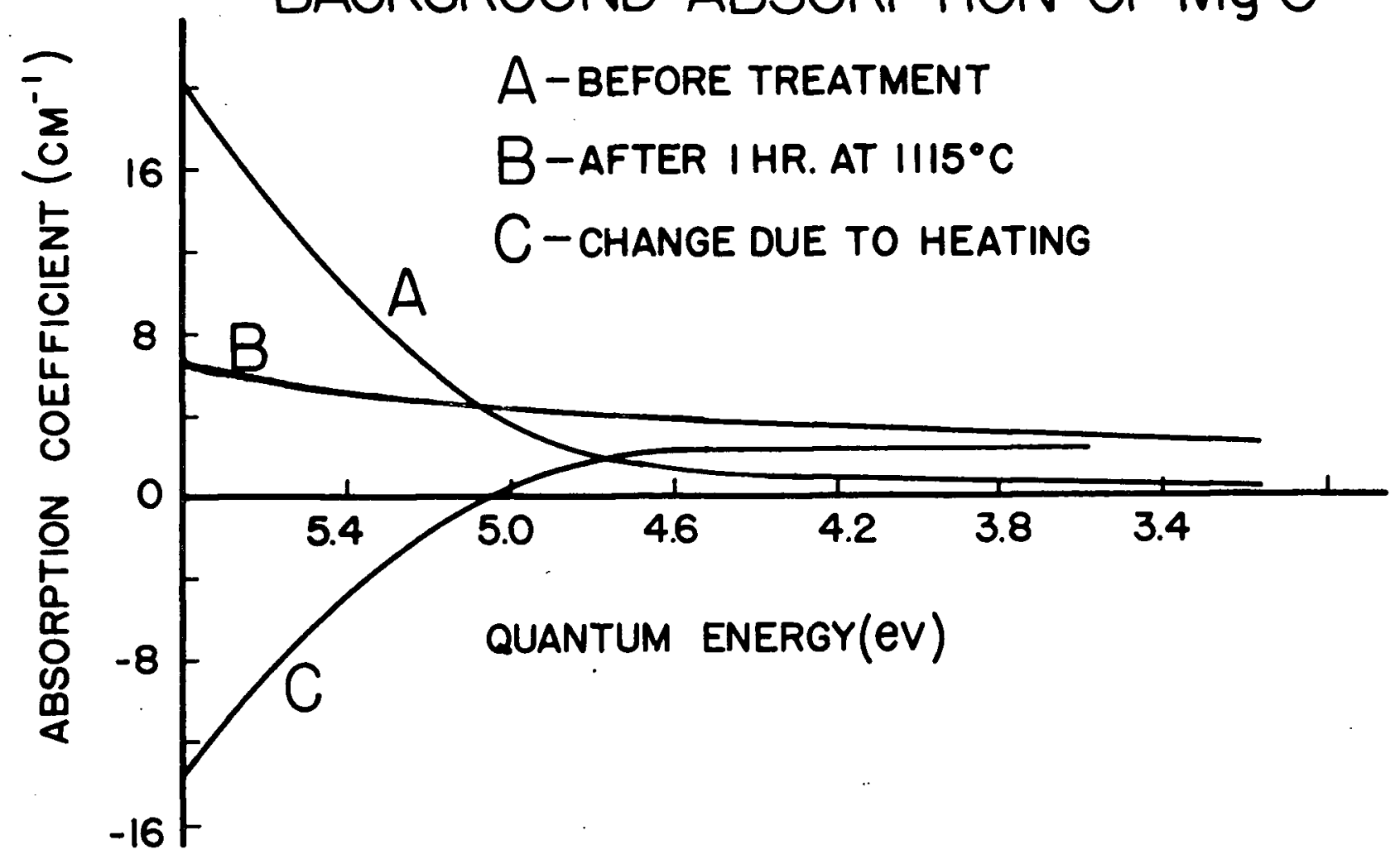

FIG. 10 

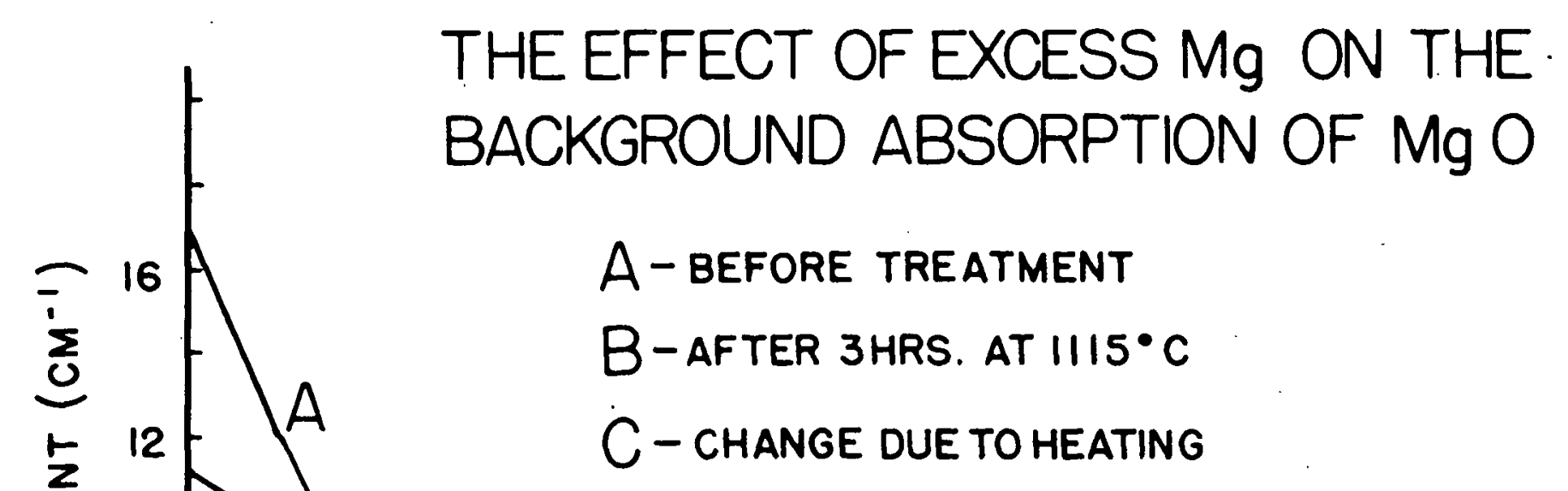


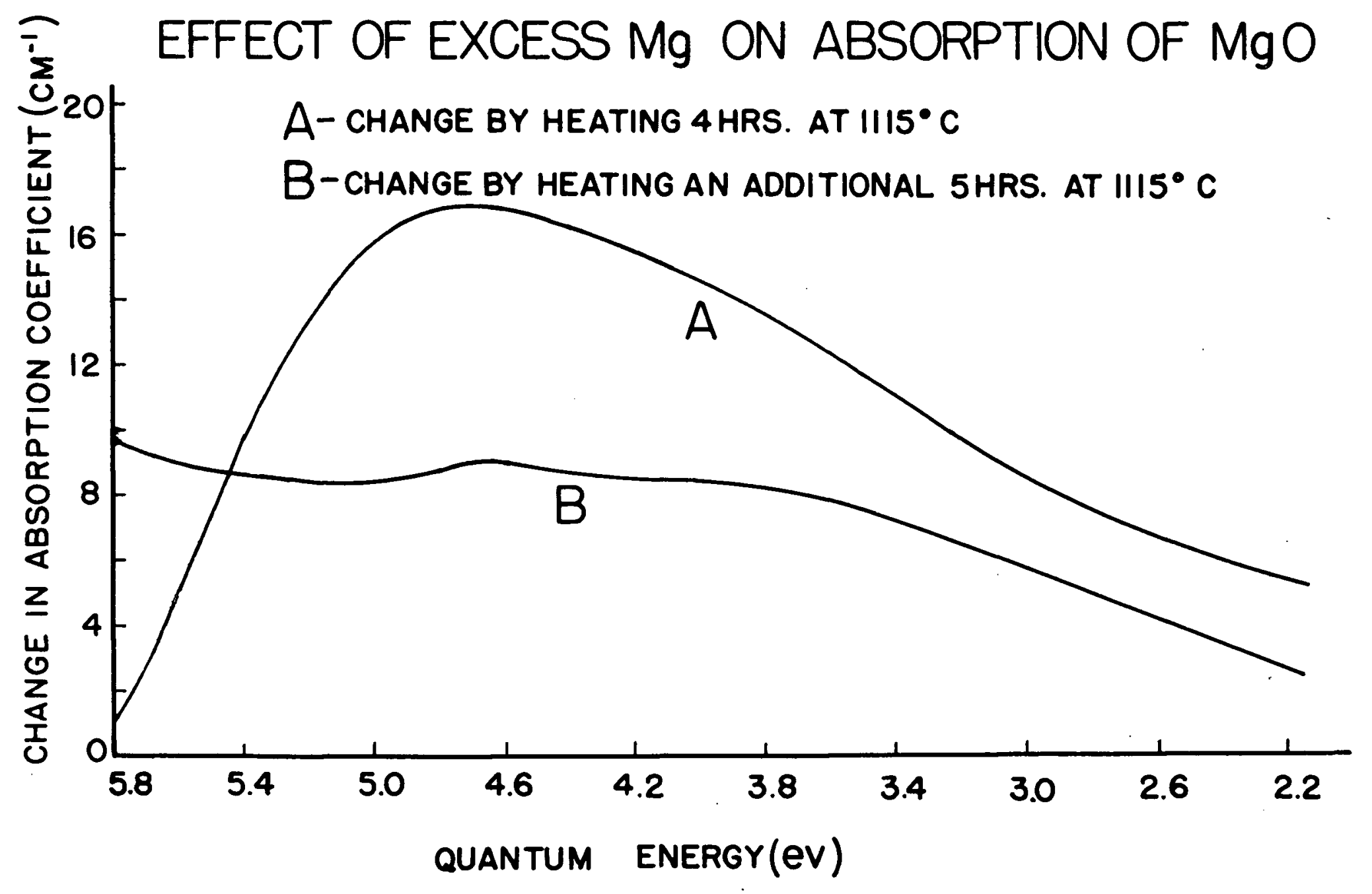

FIG. 12 


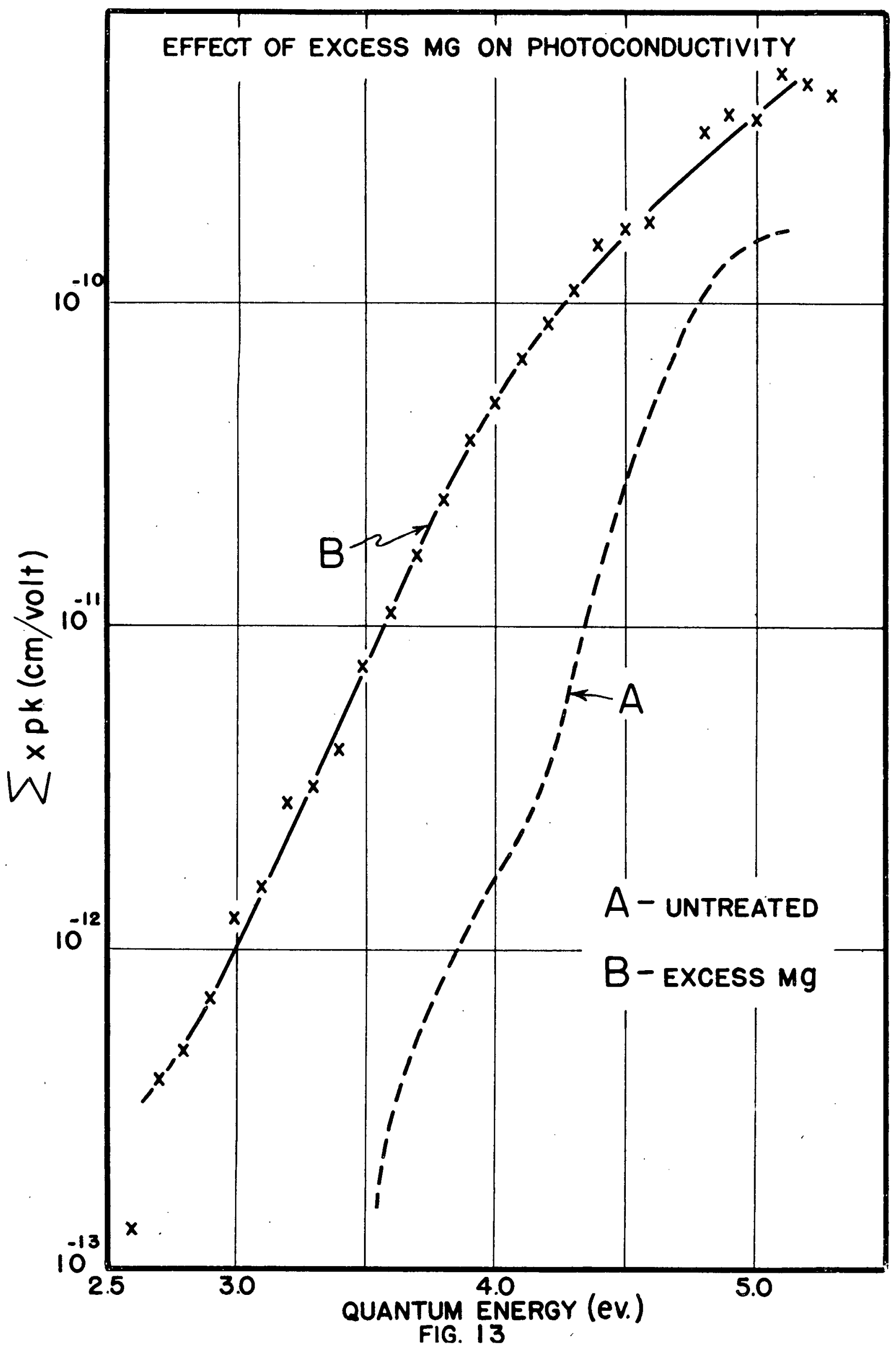




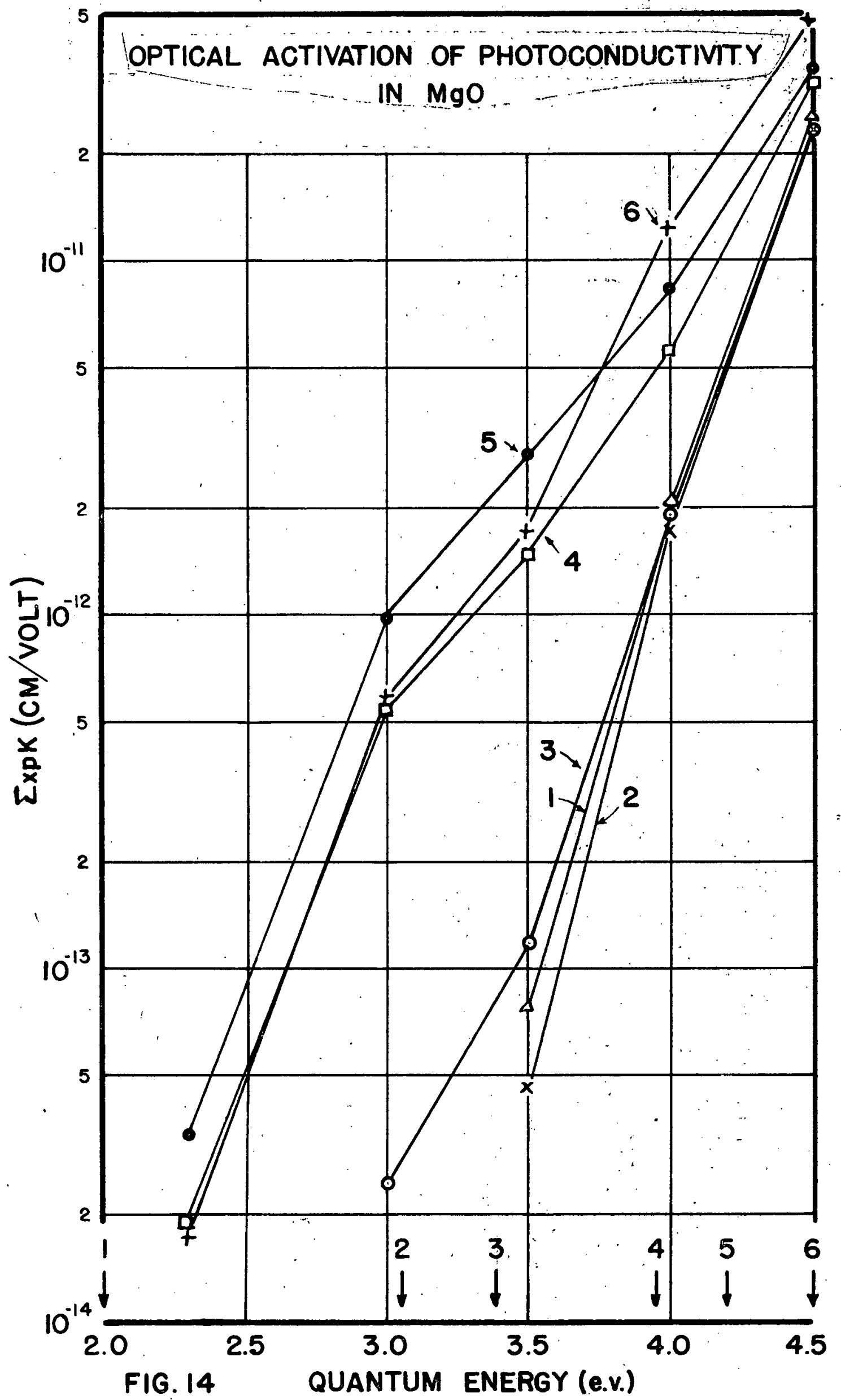




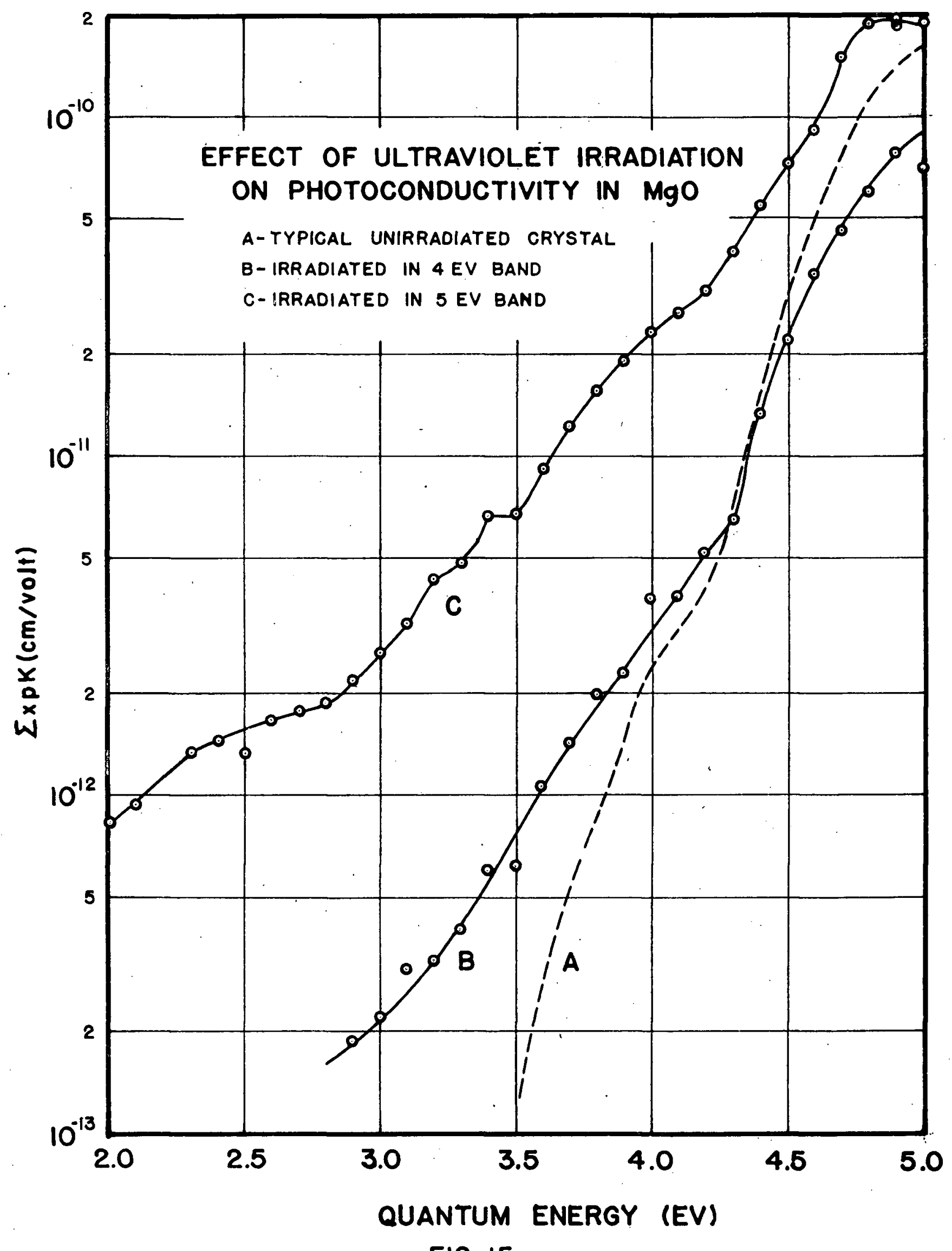

FIG. 15 


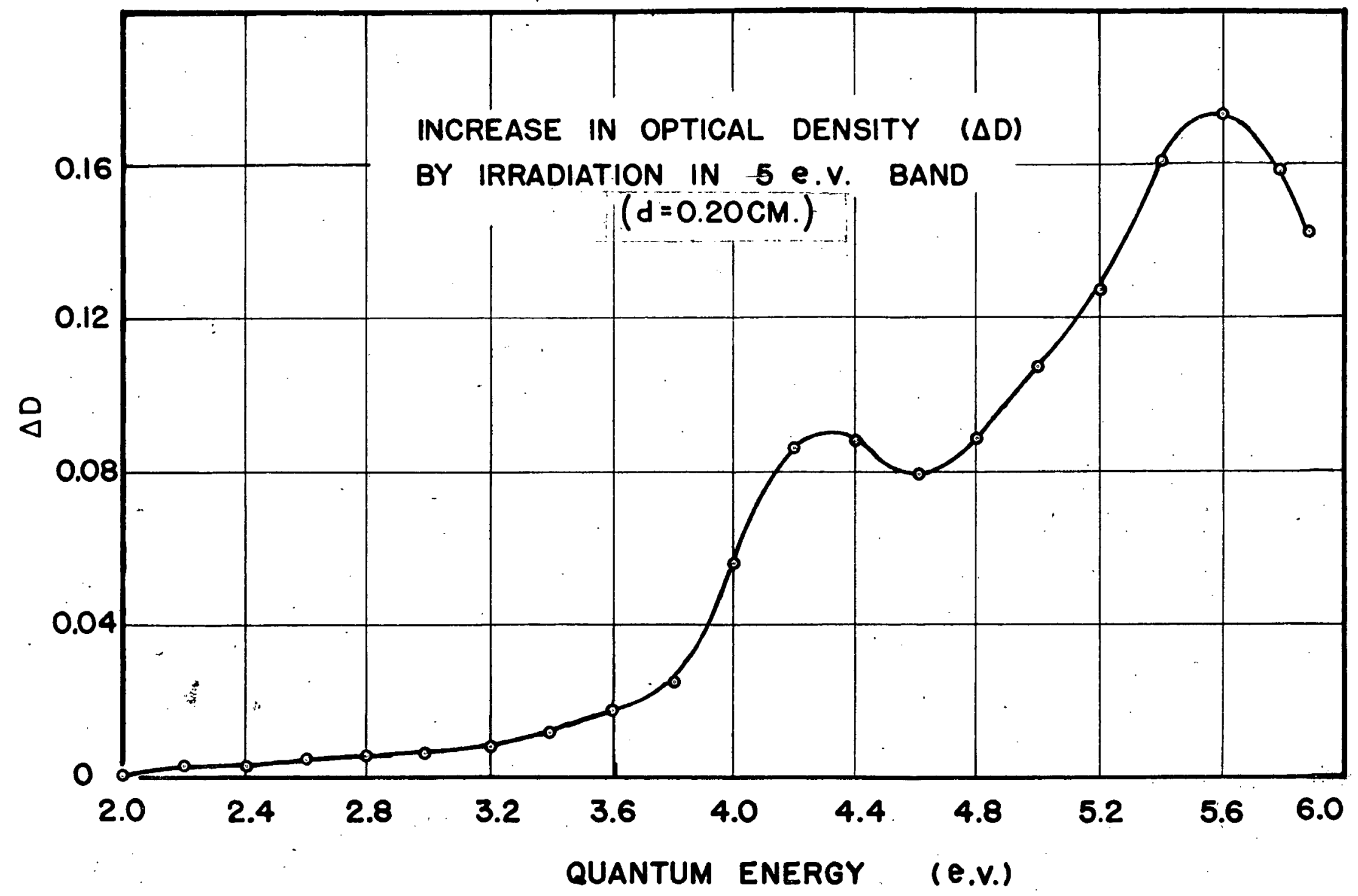

FIG. 16 


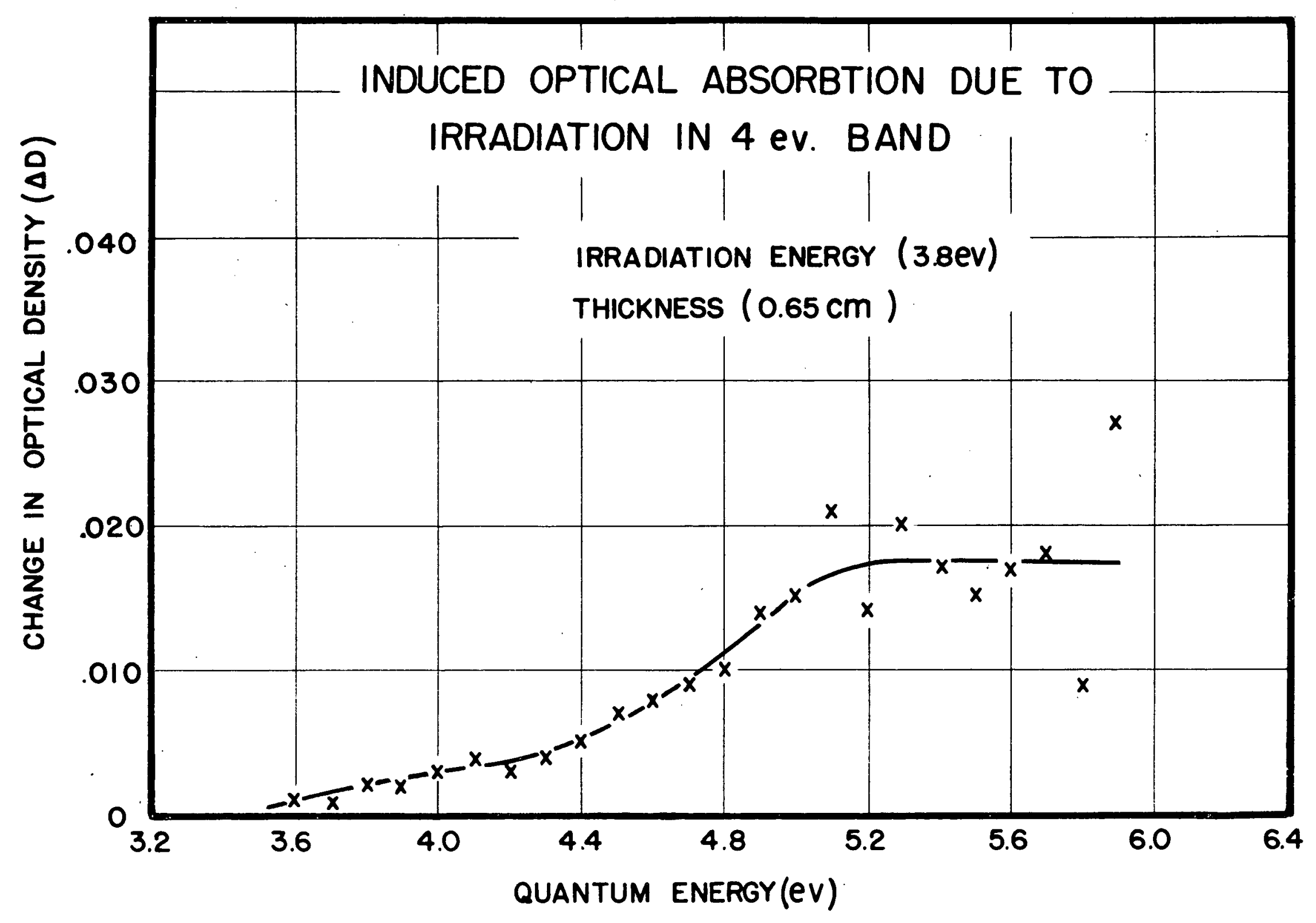

FIG. 17 


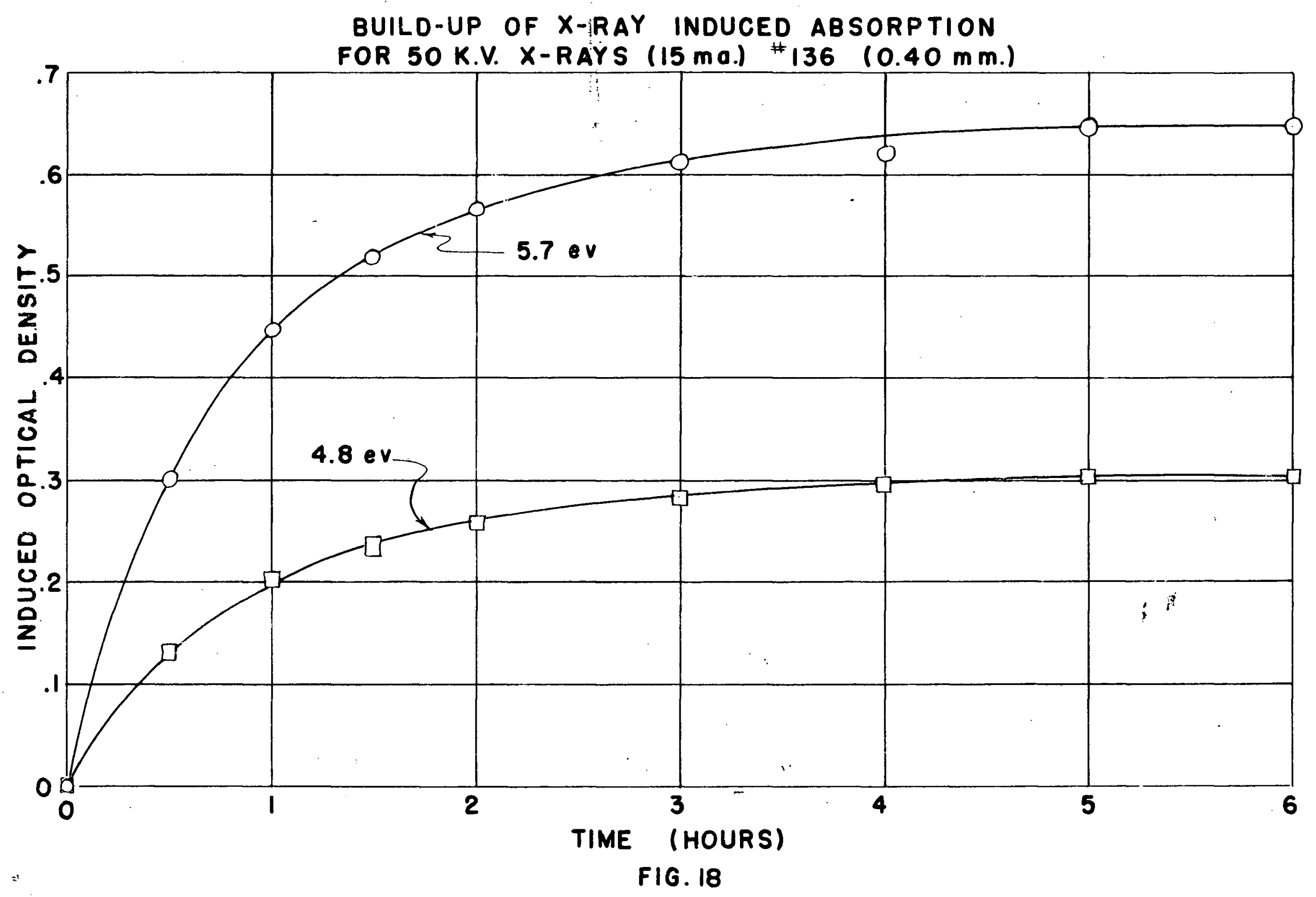




\section{ABSORPTION SPECTRA OF X-RAYED CRYSTALS}

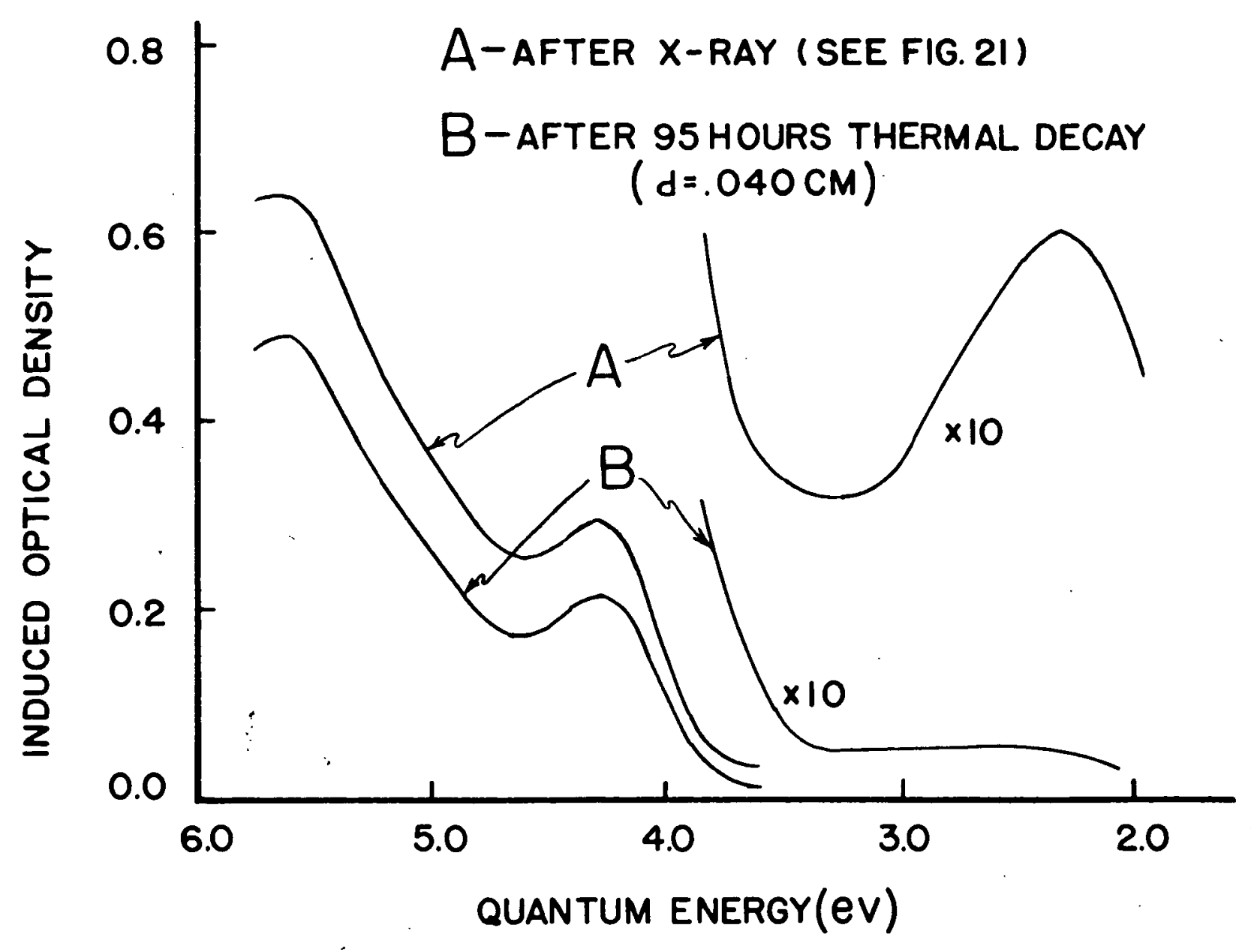

FIG. 19 


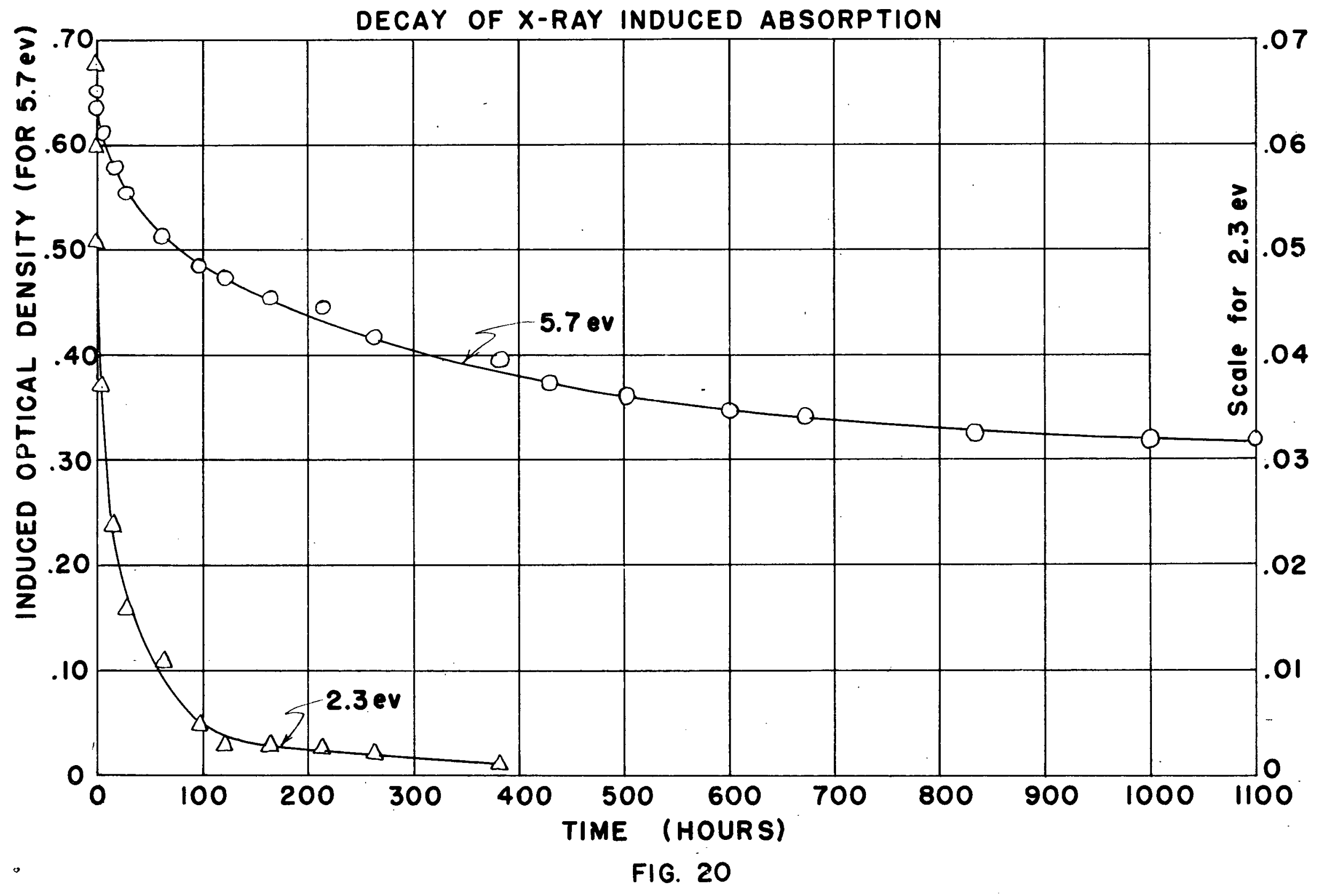




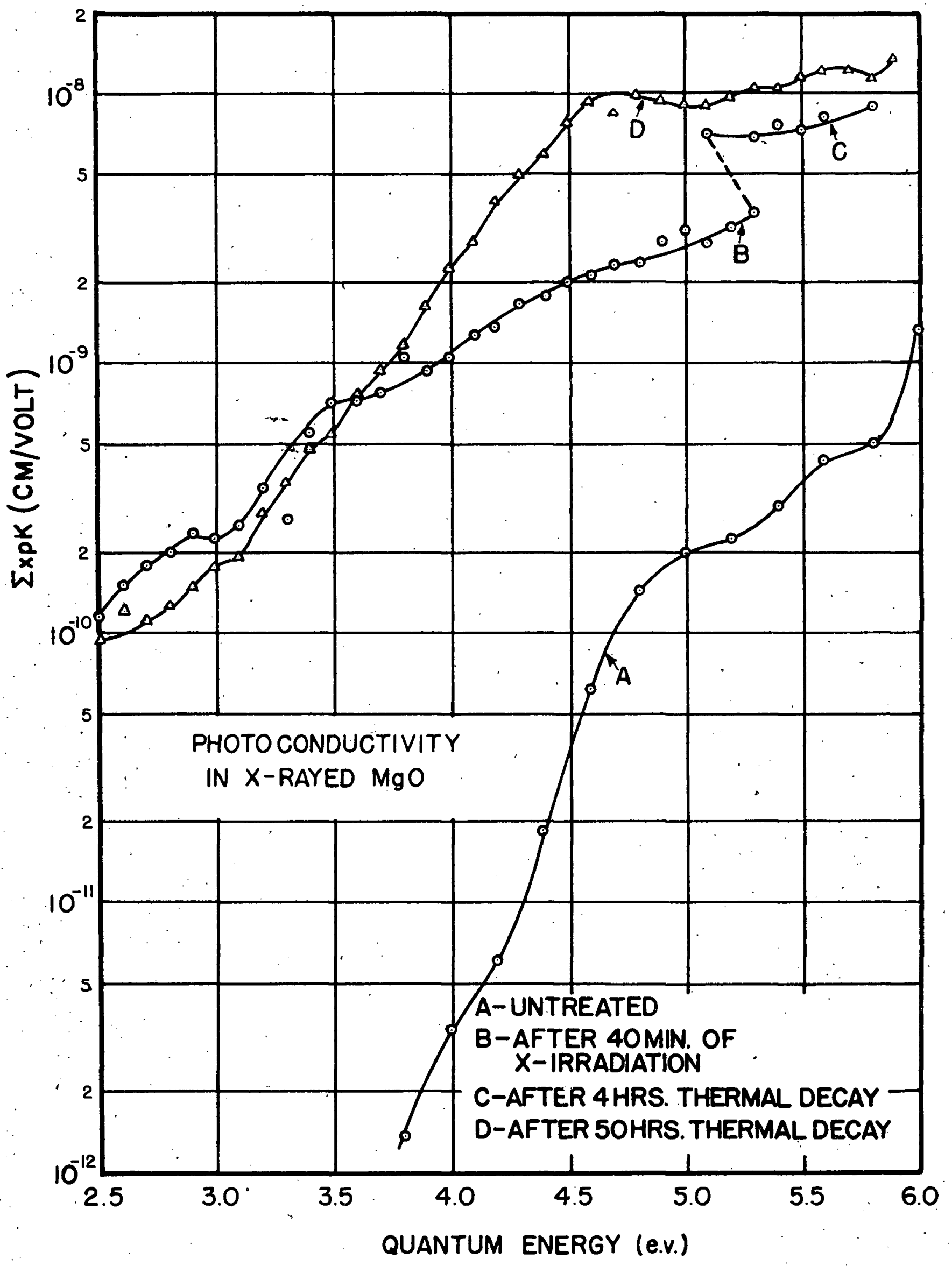

FIG. 2 I 


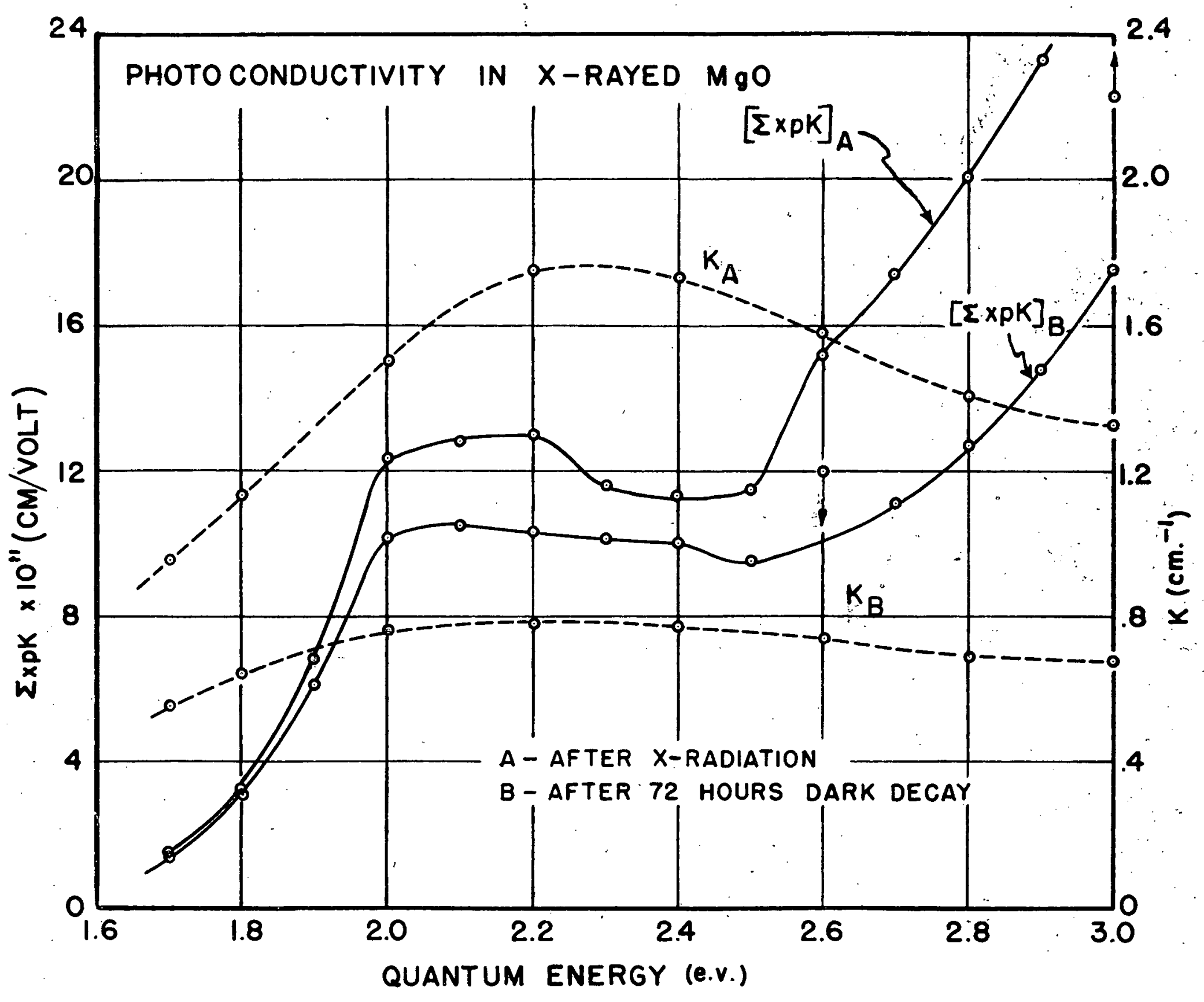

FIG. 22 


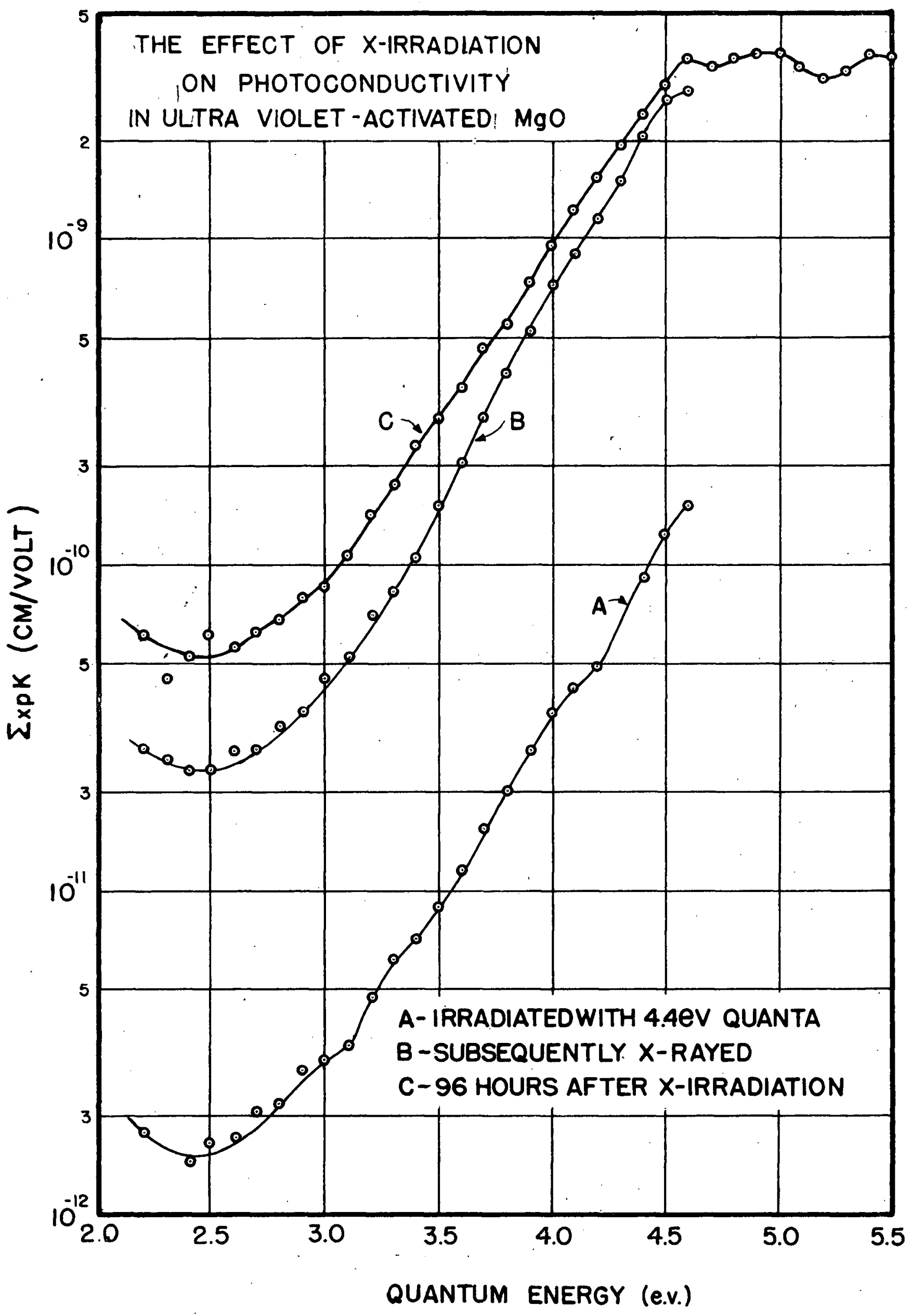

FIG. 23 


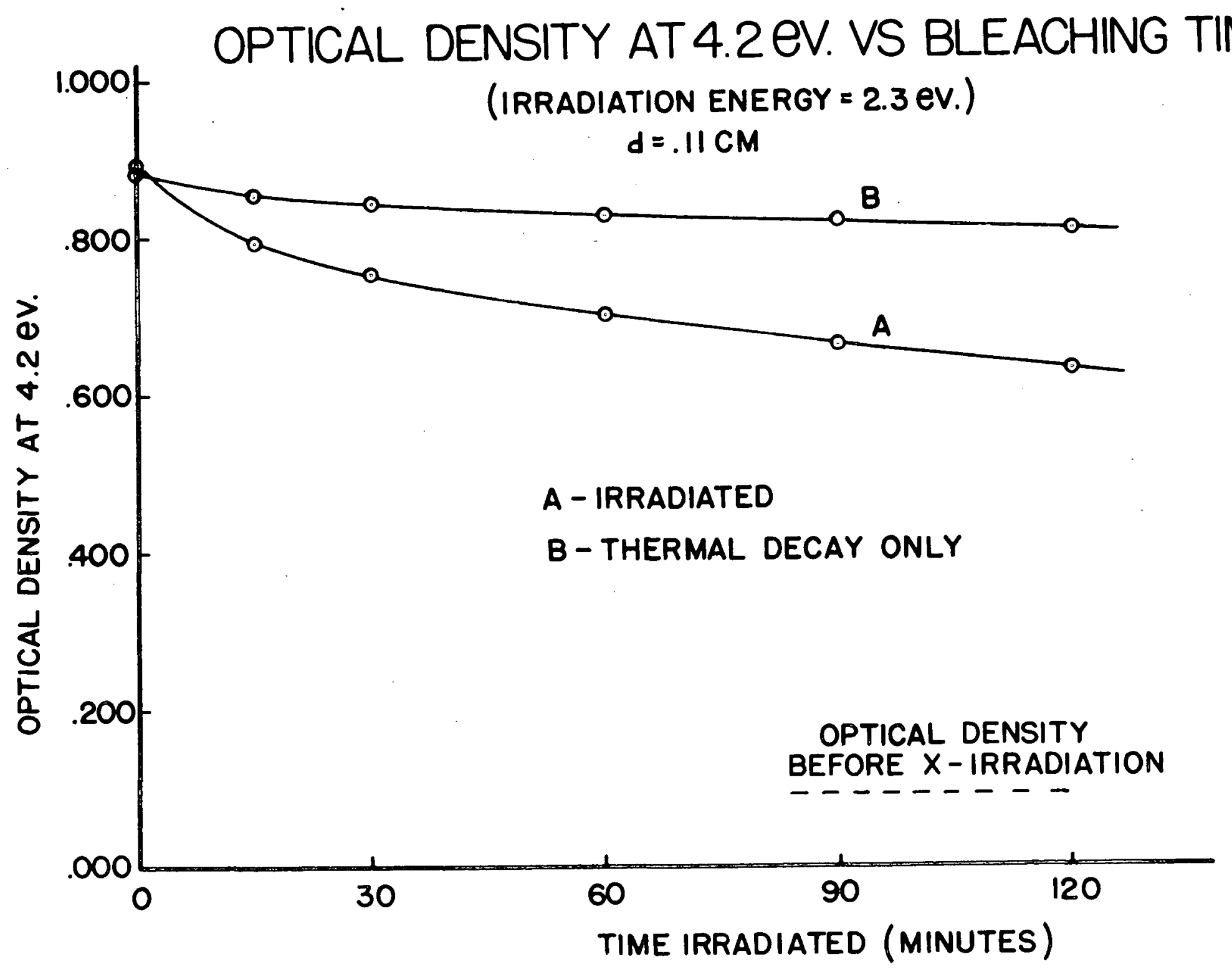

FIG. 24 


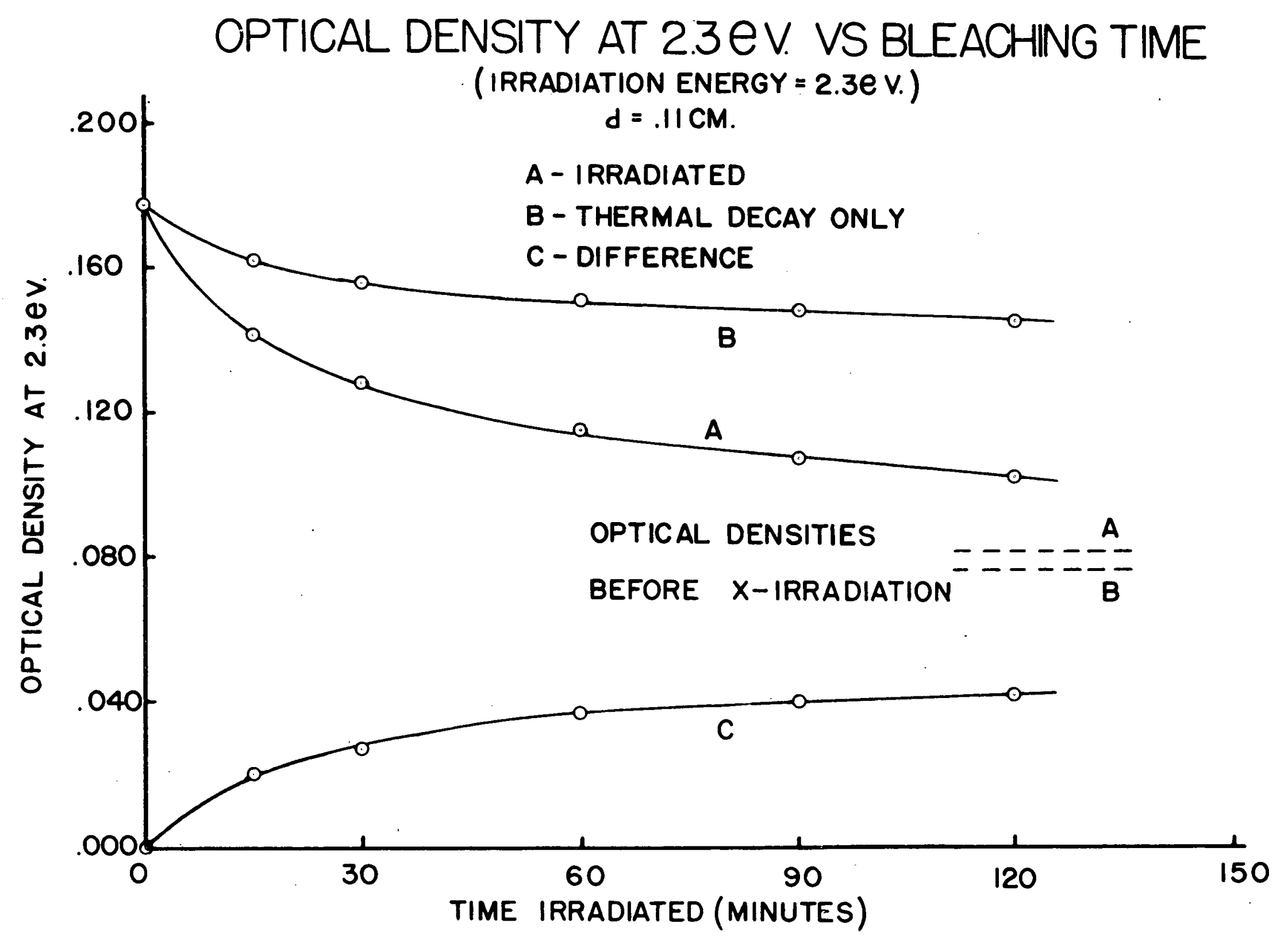

FIG. 25 


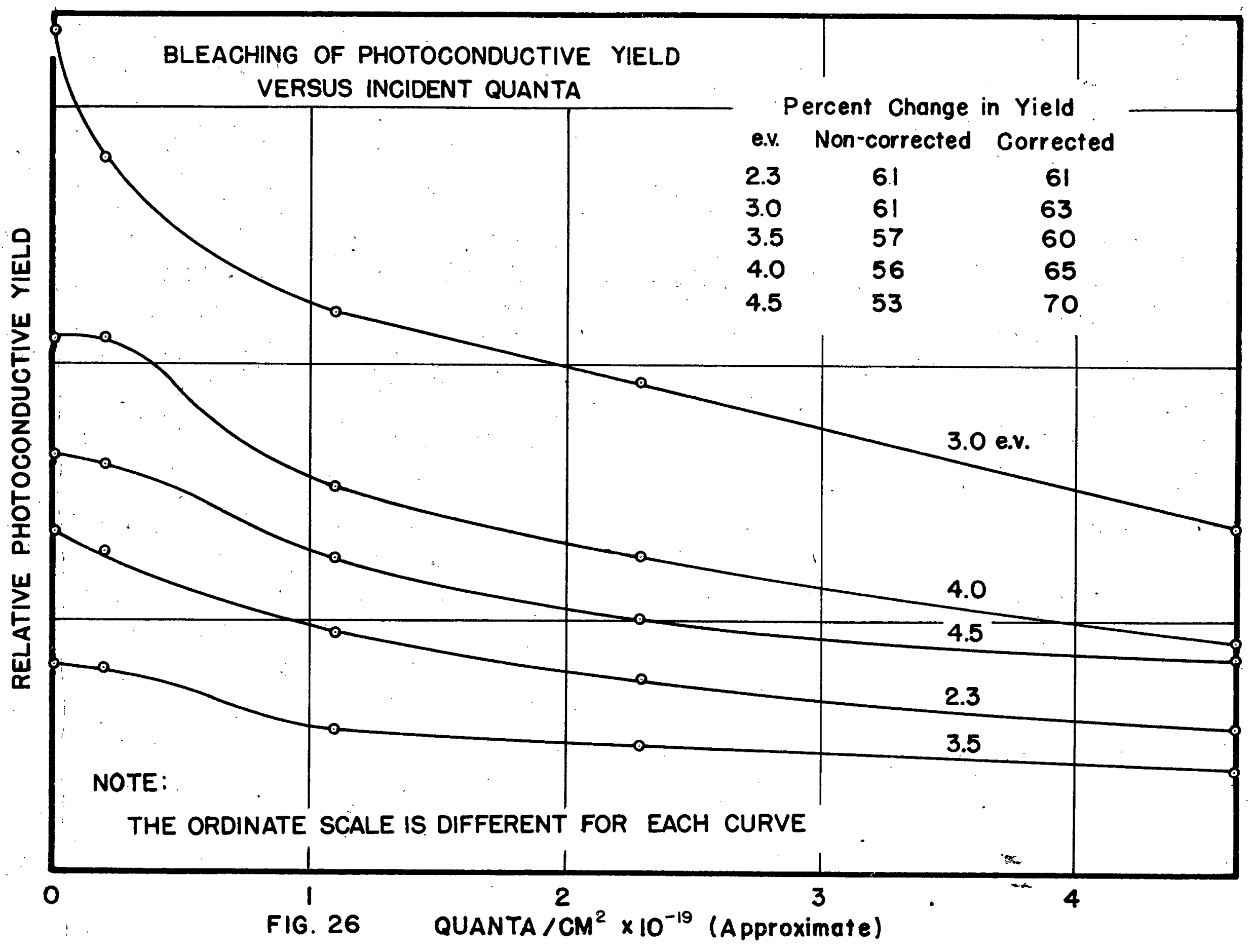


DETERMINATION OF THE SIGN OF CHARGE CARRIERS EXCITED IN 4 EV BAND

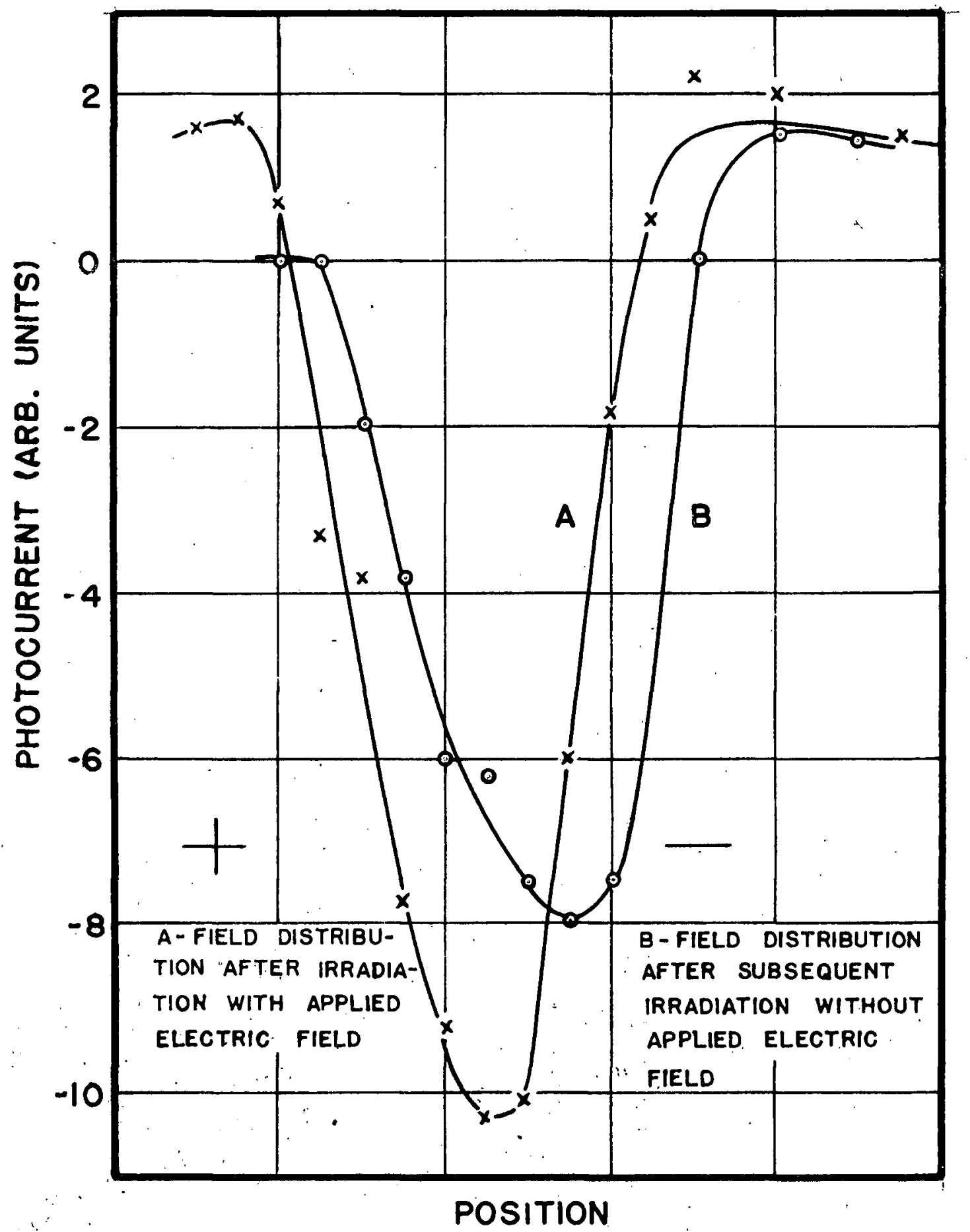

FIG. 27 
FIELD DISTRIBUTIONS AFTER IRRADIATION IN 5 EV BAND A-AFTER IRRADIATION WITH APPLIED ELECTRIC FIELD B-AFTER SUBSEQUENT IRRADIATION WITH NO APPLIED ELECTRIC FIELD C-AFTER FURTHER IRRADIATION WITH NO APPLIED ELECTRIC FIELD

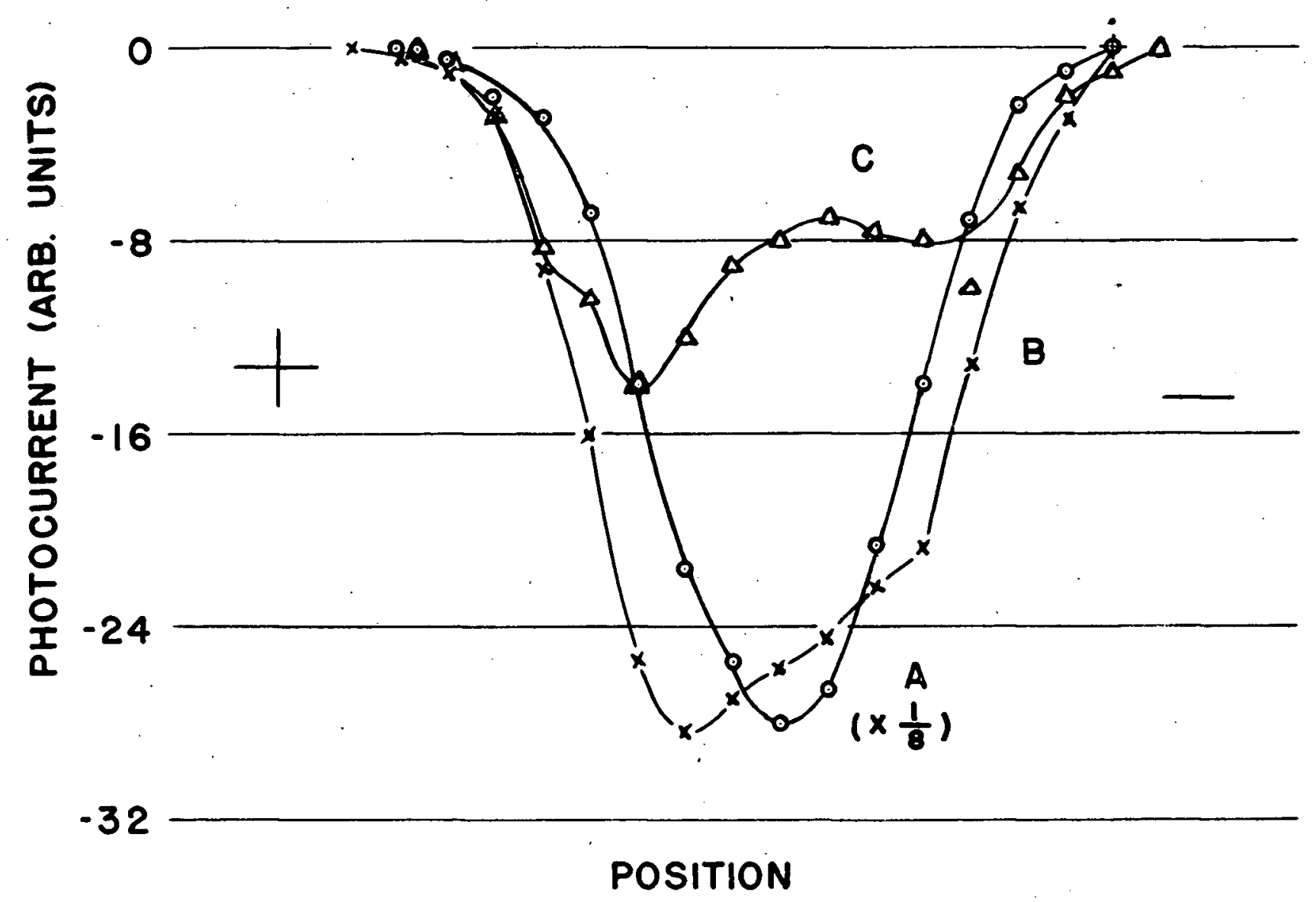

FIG. 28 


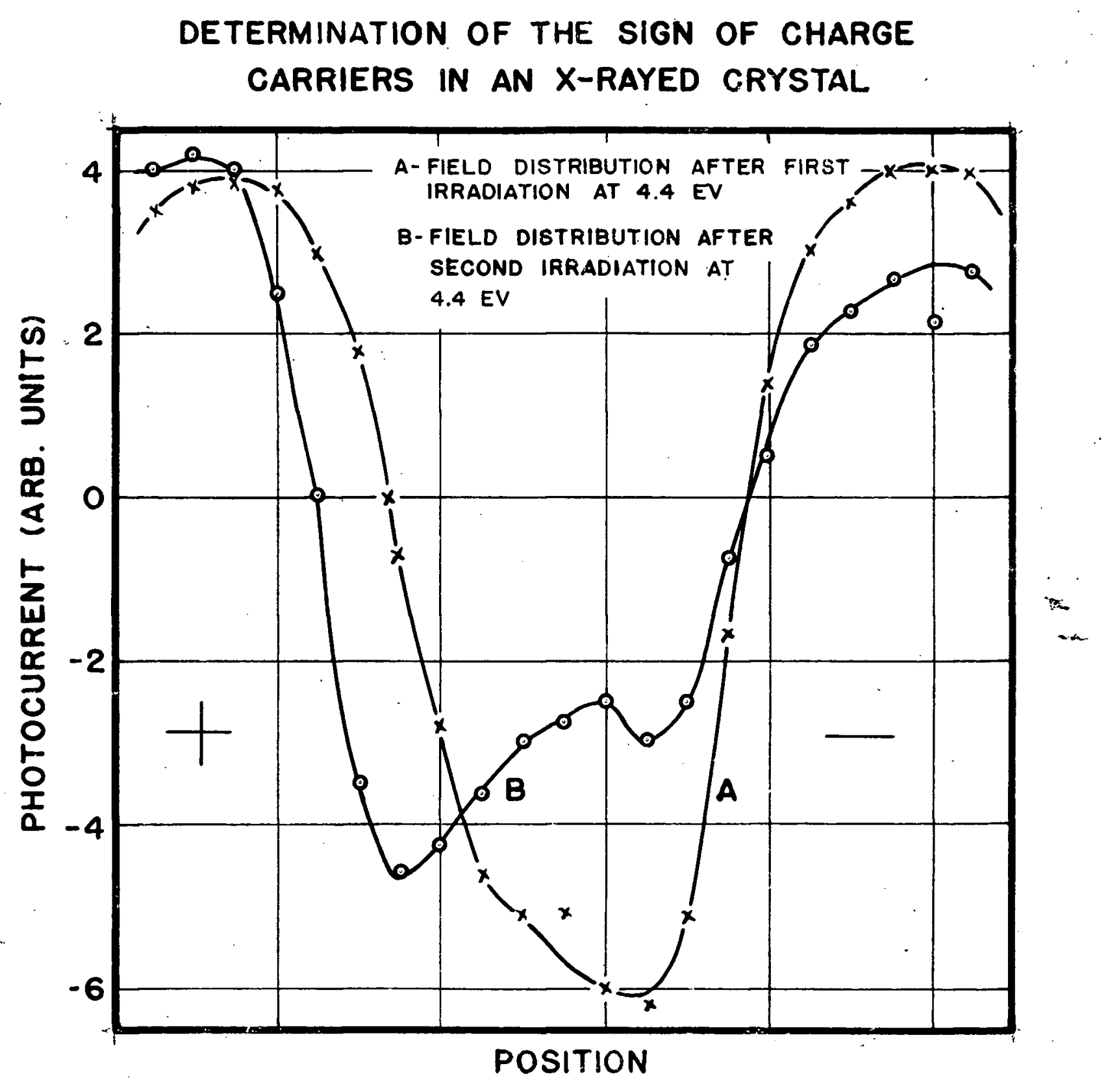

FIG. 29 


\section{POSSIBLE MECHANISMS FOR THE LOSS OF FREE CARRIERS}

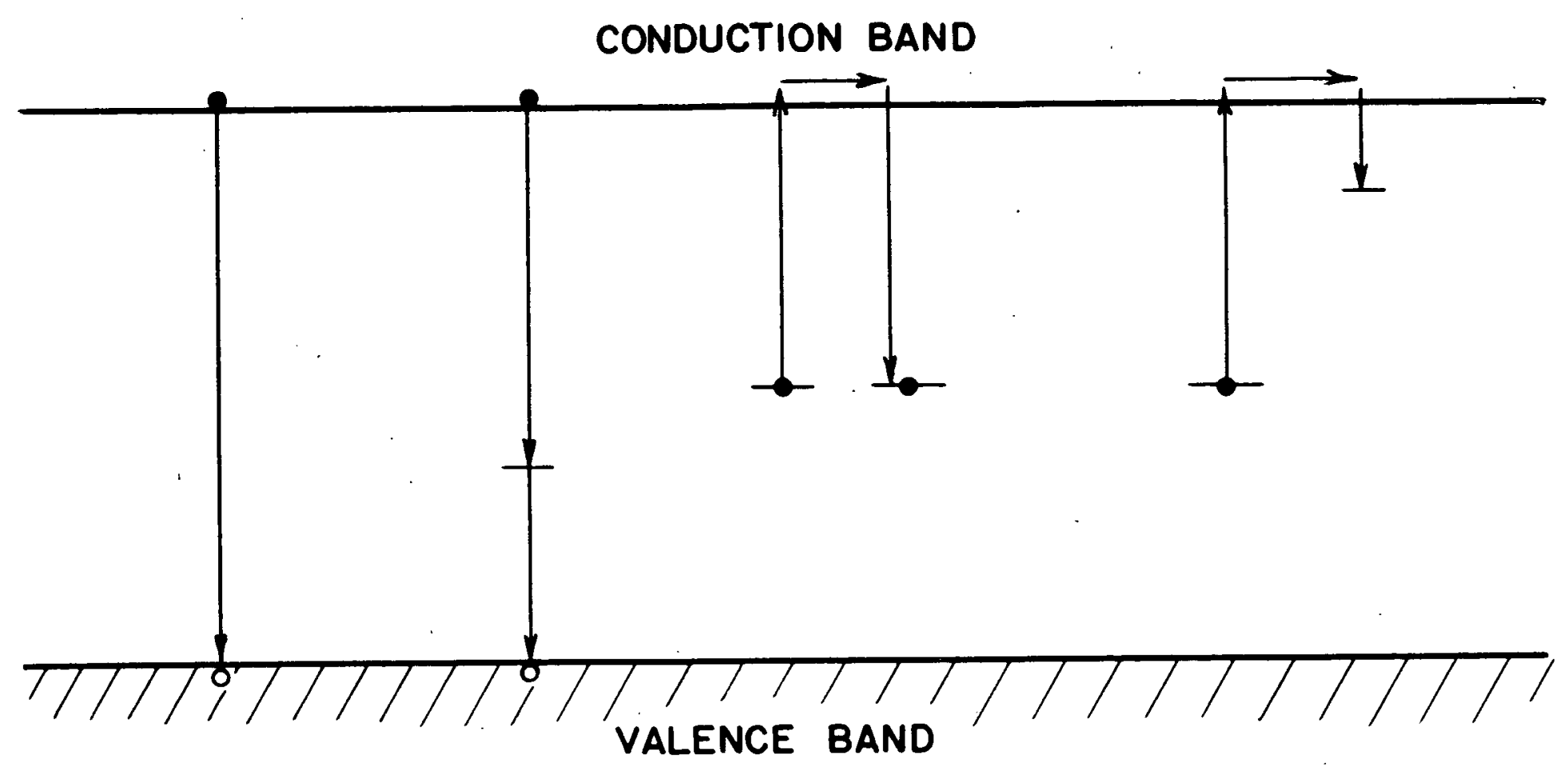
(a)
(b)
(c)
(d) 


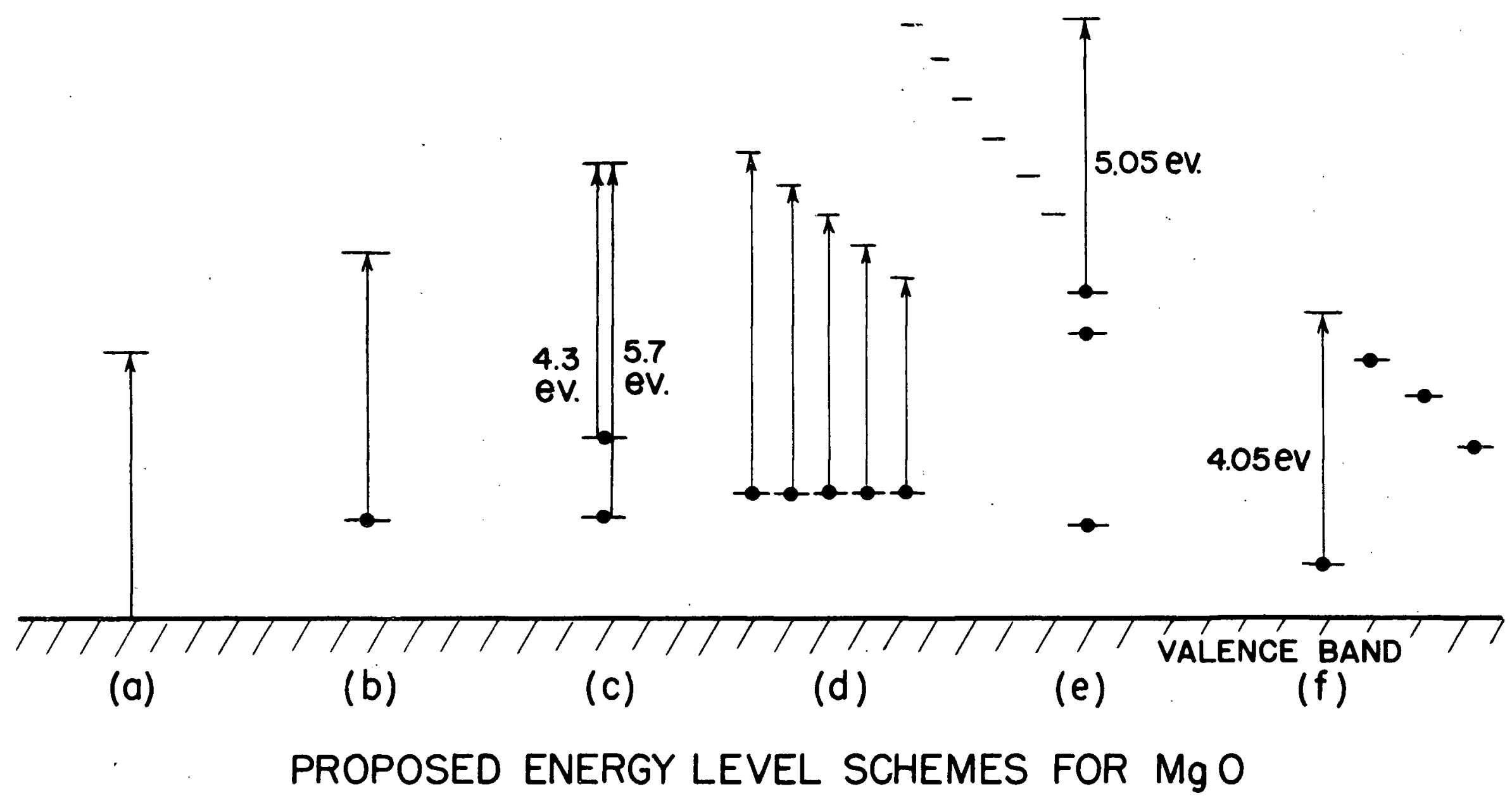

FIG. 3 I 


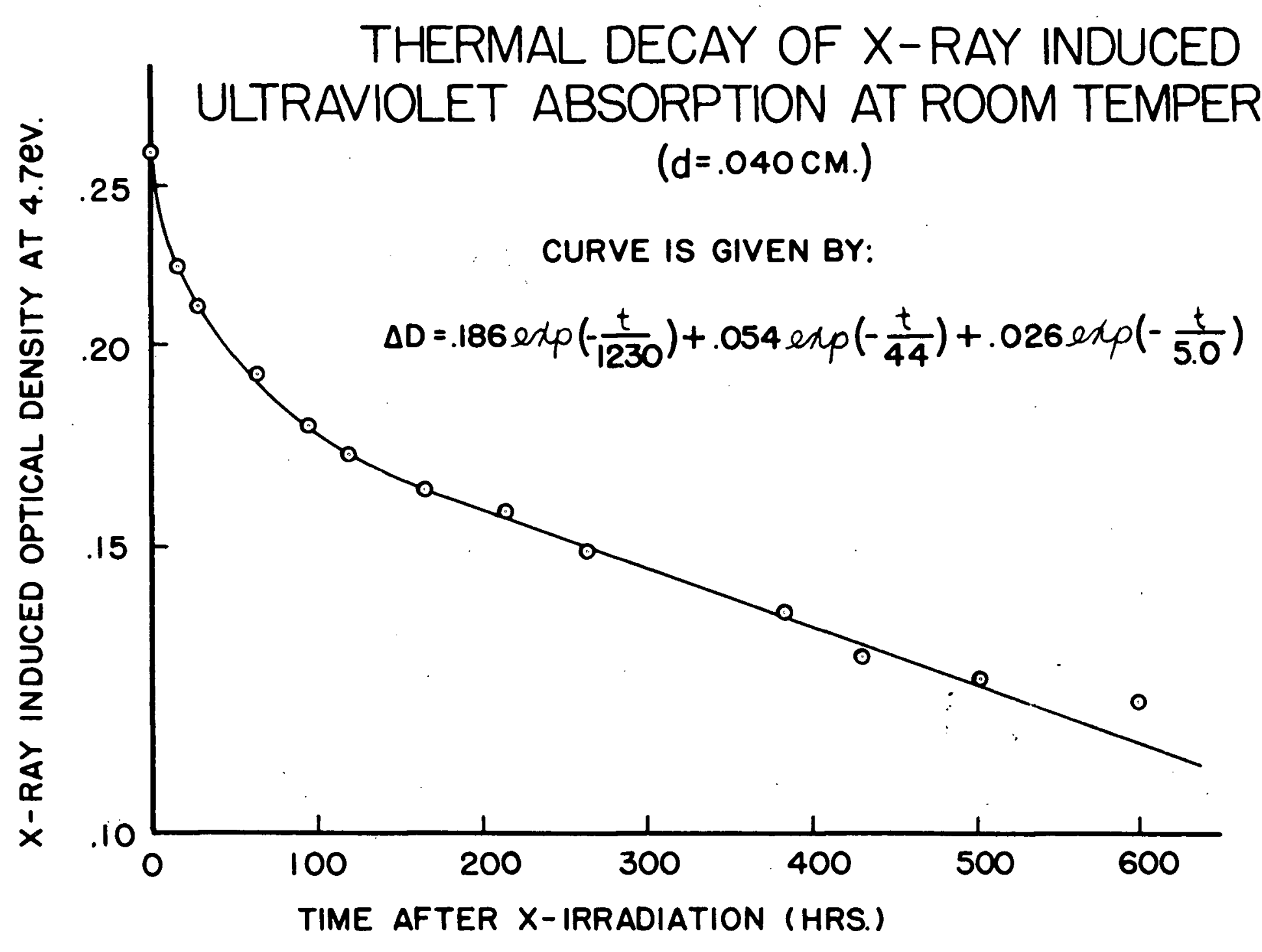

FIG. 32 
IDEALIZED FIELD DISTRIBUTIONS ILLUSTRATING THE METHOD OF DETERMINING THE SIGN OF THE CHARGE CARRIERS

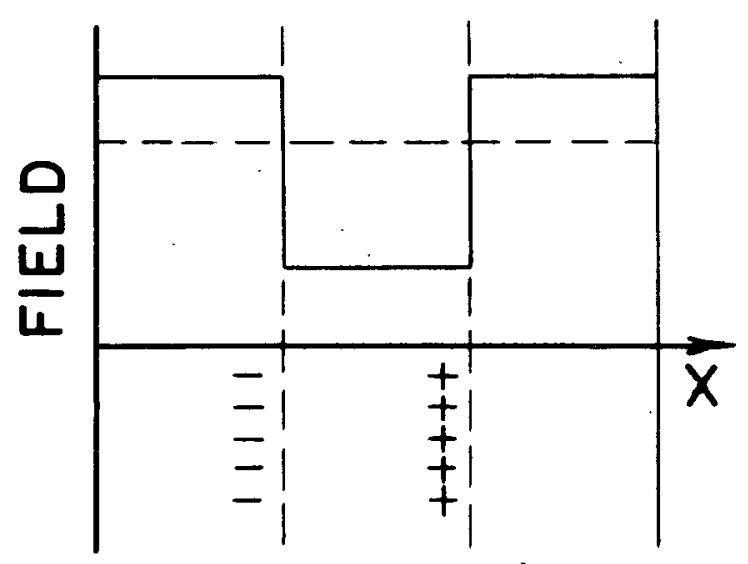

$x=0 \quad x=a \quad x=a+b \quad x=2 a+b$ POSITIVE ELECTRODE

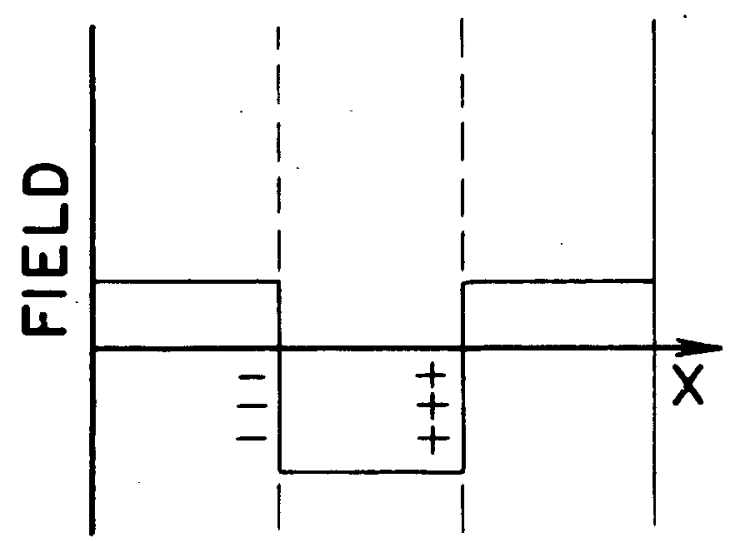

$x=0 \quad x=a \quad x=a+b \quad x=2 a+b$ NEGATIVE POSITIVE ELECTRODE ELECTRODE NEGATIVE ELECTRODE POSITIVE ELECTRODE

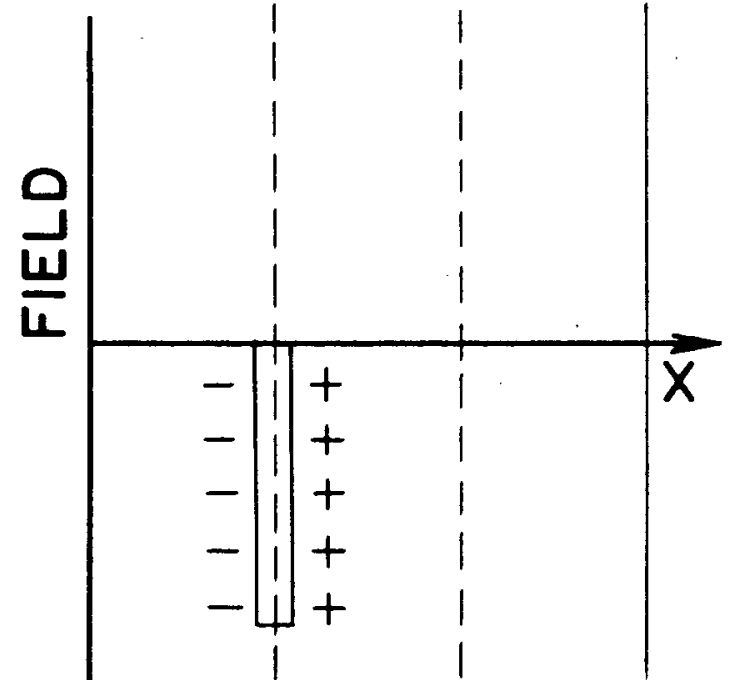

$$
x=0 \quad x=a \quad x=a+b \quad x=2 a+b
$$
NEGATIVE ELECTRODE (a) (b) 